\title{
Spherical metrics with conical singularities \\ on a 2-sphere: angle constraints
}

\author{
Gabriele Mondello ${ }^{1}$ and Dmitri Panov ${ }^{2}$ \\ 1 "Sapienza" Università di Roma, Department of Mathematics (mondello@mat.uniroma1.it) \\ ${ }^{2}$ King's College London, Department of Mathematics (dmitri.panov@kcl.ac.uk)
}

May 11, 2015

\begin{abstract}
In this article we give a criterion for the existence of a metric of curvature 1 on a 2 -sphere with $n$ conical singularities of prescribed angles $2 \pi \vartheta_{1}, \ldots, 2 \pi \vartheta_{n}$ and non-coaxial holonomy. Such a necessary and sufficient condition is expressed in terms of linear inequalities in $\vartheta_{1}, \ldots, \vartheta_{n}$.
\end{abstract}

\section{Contents}

1 Introduction

1.1 Formulation of the problem $\ldots \ldots \ldots \ldots$

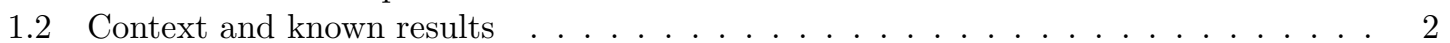

1.3 Main results . . . . . . . . . . . . . . . . . . 3

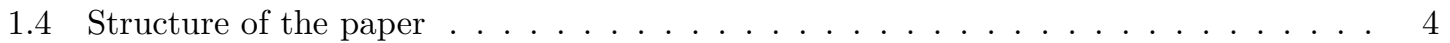

1.5 Acknowledgements . . . . . . . . . . . . . . . . . . 5

2 Algebraic constraints 5

2.1 Holonomy constraints . . . . . . . . . . . . . . . . . . . . 5

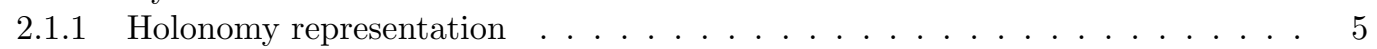

2.1 .2 Canonical lift to $\mathrm{SU}(2) \ldots \ldots \ldots \ldots \ldots \ldots$

2.1.3 Matrices in $\mathrm{SU}(2)$ and broken geodesics on $\mathbb{S}^{3} \ldots \ldots \ldots \ldots \ldots$

2.2 Algebraic merging . . . . . . . . . . . . . . . . . . . . 11

2.2.1 Intersection of $\mathcal{A}^{n}$ with a unit integer cube . . . . . . . . . . . . . . . 11

2.2.2 Simplicial and non-simplicial merging . . . . . . . . . . . . . . . . 13

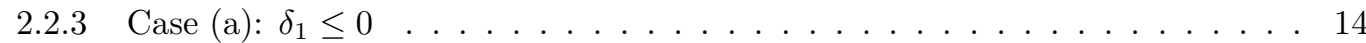

2.2 .4 Case (b): $\delta_{1}>0$ and $\delta_{2}+\delta_{3}>-1 \ldots \ldots \ldots \ldots \ldots$

2.2.5 Case (c): $\delta_{1}>0$ and $\delta_{2}+\delta_{3} \leq-1 \ldots \ldots \ldots \ldots$

3 Geometric constructions $\mathbf{1 7}$

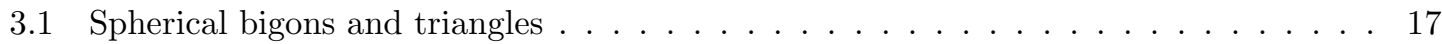

3.1 .1 Bigons . . . . . . . . . . . . . . . . . . . . . 17

3.1 .2 Triangles ......................... 18

3.1.3 Almost degenerate triangles . . . . . . . . . . . . . . . . . 22

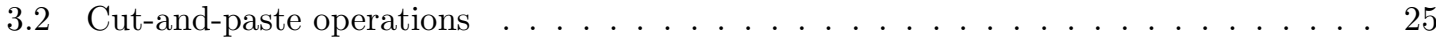

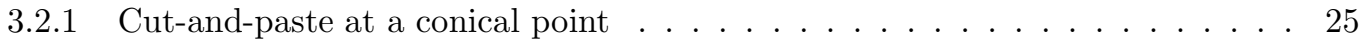

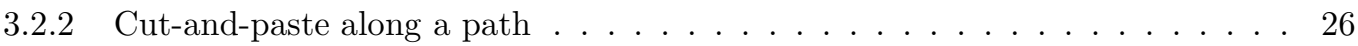

3.3 Spheres with four conical points . . . . . . . . . . . . . . . . . 27

3.3 .1 Convex quadrilaterals . . . . . . . . . . . . . . . . . 27

3.3.2 Non-convex quadrilaterals embedded in $\mathbb{S}^{2} \ldots \ldots \ldots \ldots \ldots \ldots$. . . . . . 28

3.3.3 Quadrilaterals immersed in $\mathbb{S}^{2} \ldots \ldots \ldots \ldots \ldots$. . . . . . . . . . 30

3.3.4 Sporadic families of 4 -punctured spheres . . . . . . . . . . . . . . . 31 
3.3.5 Spheres with $\vartheta_{1}, \vartheta_{2}<2, \vartheta_{3}, \vartheta_{4}<1$ and with $1<\vartheta_{1}<2, \vartheta_{2}, \vartheta_{3}, \vartheta_{4}<1 \ldots 32$

3.3.6 Existence of spheres with 4 conical points and non-integral angles . . . . . . 33

3.4 Splitting conical points . . . . . . . . . . . . . . . . . 35

List of symbols

\section{Introduction}

\subsection{Formulation of the problem}

The aim of this paper is to prove the existence of Riemannian metrics of curvature 1 with $n \geq 2$ conical singularities of assigned angles on a compact connected orientable surface of genus 0 .

In order to state the problem more formally, we recall some terminology.

Notation. By surface we always mean a smooth 2-dimensional real manifold, possibly with boundary, and we will call a sphere just a compact connected orientable surface diffeomorphic to $S^{2}$. By metric we always mean a Riemannian metric and by spherical metric just a Riemannian metric of constant curvature 1 . We keep the notation $\mathbb{S}^{2}$ for the unit 2-sphere endowed with the standard metric.

From the local point of view, spherical metrics are easy to describe: it is a classical result (see Killing [9] and Hopf [8]) that a surface endowed with a spherical metric is locally isometric to a portion of $\mathbb{S}^{2}$.

From a global point of view spherical metrics on compact connected orientable surfaces only exist in genus 0 by Gauss-Bonnet; moreover, in this case they are all isometric to each other.

The situation becomes more interesting if we allow our metrics to admit conical singularities.

Definition 1.1. A Riemannian metric $g$ of curvature 1 on a surface $S$ has a conical singularity of angle $2 \pi \alpha>0$ at $y \in S$ if it can be locally written as $g=d r^{2}+\alpha \sin (r) d \theta^{2}$, where $(r, \theta)$ are local polar coordinates on $S$ centered at $y$. We will say that the angle is integral if $\alpha \in \mathbb{Z}$.

Our goal is to answer the following question.

Question 1.2 (Existence of metrics). For which $\boldsymbol{\vartheta}=\left(\vartheta_{1}, \ldots, \vartheta_{n}\right) \in \mathbb{R}_{+}^{n}$ there exists a spherical metric $g$ on a sphere $S$ with $n$ conical singularities of angles $2 \pi \cdot \vartheta=\left(2 \pi \vartheta_{1}, \ldots, 2 \pi \vartheta_{n}\right)$ ?

\subsection{Context and known results}

We remark that Question 1.2 is actually different from the following more classical problem.

Question 1.3 (Existence of conformal metrics). Fix a connected Riemann surface $S$ with distinct points $x_{1}, \ldots, x_{n}$. For which $\boldsymbol{\vartheta}=\left(\vartheta_{1}, \ldots, \vartheta_{n}\right) \in \mathbb{R}_{+}^{n}$ there exists a conformal metric of constant curvature on $S$ with conical singularity at $x_{i}$ of angle $2 \pi \vartheta_{i}$ ?

Notice that the curvature of the desired metric in Question 1.3 must have the same sign as $\chi(S, \boldsymbol{\vartheta}):=\chi(S)+\sum_{i}\left(\vartheta_{i}-1\right)$.

Remark 1.4. Both problems described above can be formulated in terms of moduli spaces of metrics $\mathfrak{M e t}(S, x, \boldsymbol{\vartheta})$ of constant curvature on the surface $S$ with conical singularities $x_{1}, \ldots, x_{n}$ of assigned angles $2 \pi \cdot \boldsymbol{\vartheta}$ (up to isotopies that fix the singularities). Thus, Question 1.2 can be rephrased in terms of non-emptiness of $\operatorname{such} \mathfrak{M e t}(S, x, \boldsymbol{\vartheta})$; on the other hand, Question 1.3 asks whether the map $\operatorname{Met}(S, x, \boldsymbol{\vartheta}) \rightarrow \mathfrak{T}(S, x)$ to Teichmüller space that remembers the underlying conformal structure is onto. In this paper we will not push this point of view farther.

For $n=0$ and $\chi(S) \geq 0$ it is a standard fact that in every given conformal class there exists a metric of constant curvature and that such a metric is unique up to rescaling and conformal automorphisms of $S$; whereas for $\chi(S)<0$ such an existence and uniqueness statement is provided by the classical uniformization theorem proven by Koebe [10]-[11] and Poincaré [14].

Assume now $n>0$. Existence and uniqueness results were proven by Thurston [16] and Troyanov [17] for $\chi(S, \boldsymbol{\vartheta})=0$ and by McOwen [13] and Troyanov [19] for $\chi(S, \boldsymbol{\vartheta})<0$. 
As for the case $\chi(S, \boldsymbol{\vartheta})>0$, existence and uniqueness still holds in the subcritical case (and so in particular when all angles are smaller than $2 \pi$ ) again by Troyanov [19. On the other hand, it is known that such uniqueness does not hold any more in the supercritical case. For instance, an existence theorem was proven by Bartolucci-De Marchis-Malchiodi 1 for $\chi(S) \leq 0$ and a lower bound for the number of such metrics is also provided. Notice that the general case of $\chi(S, \boldsymbol{\vartheta})>0$ and $\boldsymbol{\vartheta} \notin(0,1]^{n}$ is not covered by the above works.

Another manifestation of the non-uniqueness of the solution is provided by Scherbak [15], who counted the exact number of such metrics for almost all configurations of $x_{1}, \ldots, x_{n} \in S$, when $S$ is a sphere, $\chi(S, \boldsymbol{\vartheta})>0$ and all $\vartheta_{i}$ are integers.

Back to Question 1.2, it is easy to see that the only possibility for $n=1$ is a surface isometric to the standard $\mathbb{S}^{2}$. An answer to this question is also known for $n=2$ by work of Troyanov 18 and for $n=3$ by work of Eremenko 3. A detailed analysis of spherical polygons with two non-integral angles is done in [4, and more extensively for $n=4$ in [5]; spherical quadrilaterals with three non-integral angles are studied in [6].

In this paper we will give an almost complete answer to this question for $n \geq 4$.

\subsection{Main results}

Our first main result shows that the existence of a spherical metric on a sphere $S$ with conical singularities of angles $2 \pi \cdot \boldsymbol{\vartheta}$ imposes some restrictions on the vector $\boldsymbol{\vartheta}=\left(\vartheta_{1}, \ldots, \vartheta_{n}\right)$.

Notation. Denote by $\|\cdot\|_{1}$ the standard $\ell^{1}$ norm on $\mathbb{R}^{n}$ and by $d_{1}$ the associated $\ell^{1}$ distance, and let $\mathbb{Z}_{o}^{n}$ denote the subset of odd points of $\mathbb{R}^{n}$, namely of points $\boldsymbol{m}=\left(m_{1}, \ldots, m_{n}\right)$ in $\mathbb{Z}^{n}$ such that $\|\boldsymbol{m}\|_{1}$ is odd.

Theorem A (Holonomy constraints). Suppose there exists a sphere $S$ with a spherical metric with conical singularities of angles $2 \pi \vartheta_{1}, \ldots, 2 \pi \vartheta_{n}$. Then the following inequalities hold:

$$
\left.\begin{array}{rl}
\boldsymbol{\vartheta} & >0 \\
\sum_{i=1}^{n}\left(\vartheta_{i}-1\right) & >-2
\end{array}\right\}
$$

where $\mathbf{1}=(1,1, \ldots, 1) \in \mathbb{R}^{n}$.

Moreover, if equality in $(\mathrm{H})$ is attained, then the holonomy of the metric is coaxial.

Remark 1.5. The positivity constraints $(\mathbb{P})$ follow from the positivity of the angles and the positivity of the area, via the Gauss-Bonnet theorem.

As the set of points for which the holonomy constraints ( $\mathrm{H}$ ) do not hold is the union of disjoint octahedrons, we also have the following.

Lemma B (Connectedness). The set of points in $\mathbb{R}^{n}$ that strictly satisfy the holonomy constraints (स) is non-empty for $n \geq 3$ and connected for $n \geq 4$. The same holds for the subset of points that satisfy the positivity constraints $(\mathrm{P})$ and the holonomy constraints $(\mathrm{H})$ strictly.

The proof of Theorem $\mathrm{A}$ consists of a few steps. We first associate to each spherical metric on $S$ with conical singularities $x_{1}, \ldots, x_{n}$ the holonomy representation $\rho$ of the free group $\pi_{1}(S \backslash$ $\left.\left\{x_{1}, \cdots, x_{n}\right\}\right)$ in $\mathrm{SO}(3, \mathbb{R})$. Then we show that, since $S$ is a sphere, such holonomy representation admits a canonical lift $\hat{\rho}$ to $\mathrm{SU}(2)$. Thus, we relate representations $\pi_{1}\left(S \backslash\left\{x_{1}, \ldots, x_{n}\right\}\right) \rightarrow \mathrm{SU}(2)$ to closed broken geodesics on $\mathbb{S}^{3}$ and we verify that the wished closed broken geodesic on $\mathbb{S}^{3}$ exists if and only if Inequalities $(\underline{\mathrm{H}})$ are satisfied. This explains the name "holonomy constraints".

A special role will be played by "generic" holonomy representations, namely whose image does not belong to a 1-parameter subgroup of $\mathrm{SO}(3, \mathbb{R})$.

Definition 1.6. A representation $\rho$ in $\mathrm{SO}(3, \mathbb{R})$ is coaxial if its image consists of rotations about the same axis.

The second main result of this paper is the following partial converse to Theorem $\mathrm{A}$ 
Theorem $\mathbf{C}$ (Existence of spherical metrics). Let $\vartheta_{1}, \ldots, \vartheta_{n}$ be real numbers that satisfy both the positivity constraints $(\mathbb{P})$ and the holonomy constraints $(\underline{\mathrm{H}})$ strictly. Then there exists a sphere $S$ with a spherical metric with conical points of angles $2 \pi \vartheta_{1}, \ldots, 2 \pi \vartheta_{n}$ and non-coaxial holonomy.

Remark 1.7. The cases that are not covered by this theorem is when $(\bar{H})$ becomes an equality, when the holonomy of such a spherical metric is necessarily coaxial (provided such a metric exists!).

In order to prove Theorem C, we proceed as follows.

First we construct such metrics for $n=2,3,4$ (the cases $n=2$ and $n=3$ were previously treated by Troyanov and Eremenko respectively).

The idea is then to inductively produce metrics with $n \geq 5$ conical points close to degenerate ones by picking a spherical metric with fewer singularities and splitting a conical point. More precisely, given $\vartheta_{1}, \ldots, \vartheta_{n}$ as in Theorem $\mathrm{C}$ we show that the wished spherical metric on $S$ can be obtained starting from a spherical metric on $S^{\prime}$ with $n-1$ conical singularities by operating a surgery in a neighbourhood of a conical point. Typically, the surgery will produce two points of angles $2 \pi \vartheta_{i}$, $2 \pi \vartheta_{j}$ very close to each other on $S$ out of a single conical point of angle $2 \pi\left(\vartheta_{1} \pm \vartheta_{j}-1+\eta\right)$ on $S^{\prime}$, where $\eta$ is a small fee that we have to pay for the performed cut-and-paste operation. In order to take care of this little $\eta$, we use a deformation result of Luo [12.

Remark 1.8. The presence of such a possibly nonzero $\eta$ is what forces us to require that the holonomy constraints $(\underline{\mathrm{H}})$ are satisfied strictly.

Finally, the combinatorial result that tells us which conical point to split is the following.

Theorem $\mathbf{D}$ (Algebraic merging). Assume $n \geq 5$. Let $\vartheta_{1}, \ldots, \vartheta_{n}$ be real numbers that satisfy both the positivity constraints $(\mathrm{P})$ and the holonomy constraints $(\mathrm{H})$ strictly. Then there is a choice of distinct $i, j \in\{1, \ldots, n\}$ such that at least one of the following two $(n-1)$-tuples

$$
\left(\vartheta_{1}, \ldots, \hat{\vartheta}_{i}, \ldots, \hat{\vartheta}_{j}, \ldots, \vartheta_{n},\left(\vartheta_{i}+\vartheta_{j}-1\right)\right), \quad\left(\vartheta_{1}, \ldots, \hat{\vartheta}_{i}, \ldots, \hat{\vartheta}_{j}, \ldots, \vartheta_{n},\left(\vartheta_{i}-\vartheta_{j}-1\right)\right)
$$

satisfies positivity constraints and holonomy constraints strictly.

\subsection{Structure of the paper}

The paper is divided into two parts: the former deals with the holonomy constraints and the latter provides the actual geometric constructions of the wished metrics.

In Section 2.1 Theorem $\mathrm{A}$ is proven. More precisely, we first recall some well-known facts about the developing map and the holonomy representation associated to a spherical metric. Then we prove that such a representation admits a natural lift to $\mathrm{SU}(2)$ and that such a lift is canonical, if we are working with a sphere. In the remaining subsections we show that representations in $\mathrm{SU}(2)$ carry (almost) the same information as closed broken geodesics on $\mathbb{S}^{3}$, whose existence is equivalent to the holonomy constraints $(\underline{H})$. The case of Abelian and coaxial holonomy is briefly discussed.

In Section 2.2 the holonomy and positivity constraints are studied from an algebraic point of view and Lemma $\mathrm{B}$ and Theorem $\mathrm{D}$ are proven.

Section 3.1 is devoted to the study of spherical bigons and triangles. As our final goal is to prove an existence theorem, we do not try to state a full characterization of them (which can be found in [18] and [3]), but we only provide constructions. The last subsection deals with triangles which are close to a bigon or a union of two bigons: these will be the key ingredients for operating the surgery that splits a conical point.

Section 3.2 is rather short and presents three typical cases of surgery. The first one takes place near a conical point and will be used to split a conical singularity. The second and the third one are performed along a path and will be used to produce spheres with angles $2 \pi\left(\boldsymbol{\vartheta}+\boldsymbol{e}_{\boldsymbol{i}}+\boldsymbol{e}_{\boldsymbol{j}}\right)$ starting from spheres with angles $2 \pi \cdot \boldsymbol{\vartheta}$.

Spheres with four conical points are constructed in Section 3.3. Most of them can be obtained by doubling a spherical (convex and non-convex) quadrilateral. Two sporadic one-parameter families of metrics requires an ad-hoc treatment.

Finally, in Section 3.4 we show how to apply the previously developed tools to inductively construct all desired metrics with $n \geq 5$ conical points and so to prove Theorem C. 


\subsection{Acknowledgements}

This paper represents the first output of our joint effort throughout a few years. G.M. would like to thank Rafe Mazzeo for interesting conversations on this topic.

The research of G.M. was partially funded by FIRB 2010 national grant "Low-dimensional geometry and topology" (code: RBFR10GHHH_003). D.P. is a Royal Society University Research Fellow.

\section{Algebraic constraints}

\subsection{Holonomy constraints}

\subsubsection{Holonomy representation}

We recall the following well-known fact.

Lemma 2.1 (Developing simply connected surfaces). Let $\Omega$ be a connected surface endowed with a Riemannian metric of curvature 1 . Then the following hold.

(i) $\Omega$ is locally isometric to $\mathbb{S}^{2}$.

(ii) If $\Omega$ is simply connected, then these local isometries patch together to define a global developing map dev : $\Omega \rightarrow \mathbb{S}^{2}$, which is a local isometry.

(iii) Let $\tilde{p} \in \Omega$ and $\tilde{v} \in T_{\tilde{p}}^{1} \Omega$ be a unit tangent vector. If two developing maps $\operatorname{dev}, \operatorname{dev}^{\prime}: \Omega \rightarrow \mathbb{S}^{2}$ agree on $(\tilde{p}, \tilde{v})$, then they coincide on the whole $\Omega$.

Even if the surface is not simply connected, we can still develop paths on spherical surfaces.

Lemma 2.2 (Developing paths). Let $\Sigma$ be a surface with a metric of curvature 1 and let $\gamma:[0,1] \rightarrow$ $\Sigma$ be a continuous path.

(i) There exists a simply connected surface $\Omega$ with a metric of curvature 1 such that the path $\gamma$ factorizes as $\gamma=j \circ \tilde{\gamma}$, where $\tilde{\gamma}$ is a map $\tilde{\gamma}:[0,1] \rightarrow \Omega$ and $j: \Omega \rightarrow \Sigma$ is a local isometry onto its image.

(ii) The composition of $\operatorname{dev}: \Omega \rightarrow \mathbb{S}^{2}$ with $\tilde{\gamma}$ defines a developing map $\operatorname{dev}_{\gamma}=\operatorname{dev} \circ \tilde{\gamma}:[0,1] \rightarrow \mathbb{S}^{2}$ for $\gamma$. Similarly, the composition of $d(\mathrm{dev})$ and $d \tilde{\gamma}$ induce a $d\left(\operatorname{dev}_{\gamma}\right): \gamma^{*} T_{\Sigma} \rightarrow T_{\mathbb{S}^{2}}$.

The surface $\Omega$ should be thought of as a thickening of $\gamma$ : for instance, if $\gamma$ is an embedding, we can choose $j$ to be the inclusion of a tubular neighbourhood of $\gamma([0,1])$.

Proof of Lemma 2.2, Take for instance $j: \Omega \rightarrow \Sigma$ to be a universal cover and put on $\Omega$ the pullback metric from $\Sigma$. Then clearly $\gamma$ factorizes as desired and so (i) follows. Assertion (ii) is a consequence of Lemma 2.1.

In light of the previous lemma, the following is very natural.

Definition 2.3. Two paths $\gamma$ on $S$ and $\gamma^{\prime}$ on $S^{\prime}$ are isometric if their developing maps $\operatorname{dev}_{\gamma}$ and $\operatorname{dev}_{\gamma^{\prime}}$ agree up to an isometry of $\mathbb{S}^{2}$.

Now we want to attach an element of $\mathrm{SO}(3, \mathbb{R})$ to every based loop on $\Sigma$. Fix a basepoint $p \in \Sigma$ and a unit tangent vector $v \in T_{p}^{1} \Sigma$. Choose also a point $\bar{p} \in \mathbb{S}^{2}$ and a $\bar{v} \in T_{\bar{p}}^{1} \mathbb{S}^{2}$.

For every $\gamma$ loop on $\Sigma$ based at $p$, let $\gamma=j \circ \tilde{\gamma}$ and $\Omega$ be as in Lemma 2.2. For $t=0,1$ there is a unique unit vector $\tilde{v}_{t} \in T_{\tilde{\gamma}(t)}^{1} \Omega$ such that $d j_{\tilde{\gamma}(t)}\left(\tilde{v}_{t}\right)=v$. Moreover, there exists a unique choice of $\operatorname{dev}: \Omega \rightarrow \mathbb{S}^{2}$ that takes $\left(\tilde{\gamma}(0), \tilde{v}_{0}\right)$ to $(\bar{p}, \bar{v}) \in T^{1} \mathbb{S}^{2}$.

We will call $\rho(\gamma)$ the unique element of $\operatorname{SO}(3, \mathbb{R})$ that acts on $T^{1} \mathbb{S}^{2}$ by taking $d(\operatorname{dev})_{\tilde{\gamma}(0)}\left(\tilde{v}_{0}\right)=$ $(\bar{p}, \bar{v})$ to $d(\operatorname{dev})_{\tilde{\gamma}(1)}\left(\tilde{v}_{1}\right)$.

The conclusion is the following well-known fact.

Corollary 2.4 (Holonomy representation). The association $\gamma \mapsto \rho(\gamma)$ descends to a well-defined homomorphism $\rho: \pi_{1}(\Sigma, p) \rightarrow \mathrm{SO}(3, \mathbb{R})$, called holonomy representation.

Remark 2.5. $\rho$ is unique up to global conjugation, namely a different choice of $v$ and of $(\bar{p}, \bar{v})$ produce the representation $B \rho B^{-1}$ for some $B \in \mathrm{SO}(3, \mathbb{R})$. 
Remark 2.6. Given a free loop in $\Sigma$, the same construction determines a conjugacy class of elements in $\mathrm{SO}(3, \mathbb{R})$.

We will be particularly interested in the following application of Corollary 2.4.

Let $S$ be a surface homeomorphic to a sphere. Let $x_{1}, \ldots, x_{n}$ be distinct points of $S$ and let $\vartheta_{1}, \ldots, \vartheta_{n}>0$. Denote by $\dot{S}$ the punctured surface $S \backslash\left\{x_{1}, \ldots, x_{n}\right\}$.

Corollary 2.7 (Holonomy representation for cone surfaces). For every spherical metric $g$ on $\dot{S}$ with conical singularities of angles $2 \pi \vartheta_{i}$ at $x_{i}$ and for every $p \in \dot{S}$, the induced holonomy representation $\rho: \pi_{1}(\dot{S}, p) \rightarrow \mathrm{SO}(3, \mathbb{R})$ is well-defined up to global conjugation. Moreover, if $\gamma_{j}$ is a loop that simply winds around $x_{j}$, then $\rho\left(\gamma_{j}\right)$ is a rotation of angle $2 \pi \vartheta_{j}$.

In order to perform cut-and-paste constructions, we will need to establish a certain technical deformability property of spherical metrics.

Definition 2.8. A spherical metric $g$ on $\dot{S}$ with conical singularities of angles $2 \pi \cdot \vartheta=\left(2 \pi \vartheta_{1}, \ldots, 2 \pi \vartheta_{n}\right)$ is angle-deformable if there exists a neighbourhood $N$ of $\boldsymbol{\vartheta} \in \mathbb{R}^{n}$ such that the following property holds:

- There exists a continuous family of spherical metrics $\left(g_{\boldsymbol{\nu}}\right)$ on $\dot{S}$ parametrised by $\boldsymbol{\nu} \in N$ with conical singularities of angles $2 \pi \cdot \boldsymbol{\nu}$, such that $g_{\vartheta}=g$.

Notation. We say that the angle vector $\boldsymbol{\vartheta}$ or the associated defect vector $\boldsymbol{\delta}$ (see Definition 2.19) is deformable if there exists an angle-deformable metric with conical singularities of angles $2 \pi \cdot \boldsymbol{\vartheta}$.

A corollary of a theorem by Luo [12] on $\mathbb{C P}^{1}$-structures with moderate singularities can be specialized to the case of spherical metrics with non-integral angles and non-coaxial holonomy. Here we formulate it in a simplified form, well-suited to our needs.

Theorem 2.9 (Deformability). Let $\boldsymbol{\vartheta}=\left(\vartheta_{1}, \ldots, \vartheta_{n}\right)$ with each $\vartheta_{i}$ positive and non-integral. Suppose that there exists a spherical metric $g$ on $\dot{S}$ with conical singularities of angles $2 \pi \vartheta_{i}$ at $x_{i}$ and with non-coaxial holonomy. Then $g$ is angle-deformable.

On the other hand, non-coaxiality of the holonomy can be easily checked as follows.

Lemma 2.10 (Non-coaxiality criterion). Consider a spherical metric $g$ on $\dot{S}$ with conical singularities of angles $2 \pi \vartheta_{i}$ at $x_{i}$. Suppose that there exists a smooth geodesic path $\gamma$ of length $\ell \notin \pi \mathbb{Z}$ between two distinct points $x_{j}$ and $x_{k}$ such that $\vartheta_{j}, \vartheta_{k} \notin \mathbb{Z}$. Then the holonomy of $g$ is non-coaxial.

Moreover, if $\ell$ is not a multiple of $\pi / 2$ or if $\vartheta_{j}$ is not half-integral, then the holonomy of $g$ is non-Abelian.

Proof. Fix a basepoint $p \in \dot{S}$ and let $\left[\gamma_{i}\right] \in \pi_{1}(\dot{S}, p)$ be the class of a loop that simply winds about $x_{i}$. Let $\rho: \pi_{1}(\dot{S}, p) \rightarrow \mathrm{SO}(3, \mathbb{R})$ be the holonomy representation associated to the metric $g$. Since $\vartheta_{j}$ and $\vartheta_{k}$ are not integers, the transformations $\rho\left(\gamma_{j}\right)$ and $\rho\left(\gamma_{k}\right)$ have well-defined axes $A_{j}, A_{k} \subset \mathbb{R}^{3}$, which are in fact distinct because $\ell$ is not a multiple of $\pi$. Hence, the holonomy is not coaxial.

If (a) or (b) is satisfied, then $\rho\left(\gamma_{j}\right)$ does not fix $A_{k}$. This shows that $\rho\left(\gamma_{j}\right)$ and $\rho\left(\gamma_{k}\right)$ do not commute and so the holonomy is not Abelian.

Remark 2.11. It is easy to see that the only Abelian but non-coaxial holonomy representation takes values (up to conjugation) in the non-cyclic subgroup of order 4 of diagonal matrices in $\mathrm{SO}(3, \mathbb{R})$. Such a holonomy is indeed realized, for instance by a spherical surface of genus 0 with three conical points of angles $\pi$ and by suitable branched covers of it.

\subsubsection{Canonical lift to $\mathrm{SU}(2)$}

The statement of Corollary 2.7 can be slightly improved as follows.

Proposition 2.12 (Lift of the holonomy to $\mathrm{SU}(2)$ ). Let $S$ be a surface homeomorphic to a sphere and $x_{1}, \ldots, x_{n}$ be distinct points of $S$ and let $p \in \dot{S}=S \backslash\left\{x_{1}, \ldots, x_{n}\right\}$ be a basepoint. Suppose that $\dot{S}$ is endowed with a Riemannian metric of curvature 1 with conical singularities of angles $2 \pi \vartheta_{j}>0$ at $x_{j}$. Then its holonomy representation $\rho$ admits a canonical lift $\hat{\rho}: \pi_{1}(\dot{S}, p) \rightarrow \mathrm{SU}(2)$. Moreover, if $\gamma_{j}$ is a loop that winds simply around $x_{j}$, then $\hat{\rho}\left(\gamma_{j}\right)$ has eigenvalues $e^{ \pm i \pi\left(\vartheta_{j}-1\right)}$. 
Remark 2.13. Analogously as before, $\hat{\rho}$ is well-defined up to global conjugation. Moreover, a free loop in $\dot{S}$ determines a conjugacy class of elements in $\mathrm{SU}(2)$.

Remark 2.14. It is well-known that the $\operatorname{PSL}(2, \mathbb{C})$-valued holonomy representation associated to any $\mathbb{C P}^{1}$-structure on a compact Riemann surface $\Sigma$ can be lifted to $\mathrm{SL}(2, \mathbb{C})$ and that such lifts correspond to complex line bundles $L$ on $\Sigma$ such that $L^{\otimes 2} \cong T_{\Sigma}$ (see Lemma 1.3.1 in [7, for instance).

In our case, we are considering the punctured surface $\dot{S}$. Since $S$ has genus zero, requiring that $\hat{\rho}\left(\gamma_{j}\right)$ has eigenvalues $e^{ \pm i \pi\left(\vartheta_{j}-1\right)}$ already guarantees the uniqueness of the lift, so that we only have to check the existence. If we were dealing with a punctured surface $\dot{\Sigma}$ of positive genus, then the requirement on the eigenvalues of $\hat{\rho}\left(\gamma_{j}\right)$ would restore the correspondence between lifts of the holonomy representations and line bundles $L$ on $\Sigma$ such that $L^{\otimes 2} \cong T_{\Sigma}$. Since it is not needed here, we will not analyze this case.

Definition 2.15. A standard set of matrices for $\boldsymbol{\vartheta} \in \mathbb{R}_{+}^{n}$ is a $n$-uple $\left(U_{1}, \ldots, U_{n}\right)$ of elements of $\mathrm{SU}(2)$ such that $U_{1} \cdot U_{2} \cdots U_{n}=I$ and the eigenvalues of $U_{j}$ are $e^{ \pm i \pi\left(\vartheta_{j}-1\right)}$ for $j=1, \ldots, n$.

An immediate consequence of Proposition 2.12 is the following.

Corollary 2.16 (From metrics to standard matrices). Let $\vartheta_{1}, \ldots, \vartheta_{n}$ be positive real numbers. Suppose that there exists a metric of curvature 1 on a sphere $S$ with conical singularities of angles $2 \pi \vartheta_{1}, \ldots, 2 \pi \vartheta_{n}$. Then there exists a standard set of matrices for $\boldsymbol{\vartheta}$.

Fix a standard set of generators $\gamma_{1}, \ldots, \gamma_{n}$ of $\pi_{1}(\dot{S}, p)$, namely

- $\gamma_{j}:[0,1] \rightarrow \dot{S}$ is a smooth simple loop that winds counterclockwise around $x_{j}$;

- the images of $\gamma_{j}$ intersect only at $p$;

- $\gamma_{1} * \cdots * \gamma_{n} \simeq c_{p}$ the constant path at $p$.

Proof of Corollary 2.16, Just let $U_{j}:=\hat{\rho}\left(\gamma_{j}\right)$, where $\hat{\rho}$ is the canonical lift provided by Proposition 2.12 .

Though Proposition 2.12 may be phrased in the more general context of $\mathbb{C P}^{1}$-structure with conical singularities, we wish to provide a complete proof tailored to our needs in the setting of spherical metrics.

Proof of Proposition 2.12, We break the proof into three main steps.

Step 1: construction of the lift $\hat{\rho}$.

Since $S$ is homeomorphic to a sphere, there exists an open disk $D \subset S$ that contains all $x_{1}, \ldots, x_{n}$ and the images of $\gamma_{1}, \ldots, \gamma_{n}$. We can for instance assume that $S \backslash D$ consists of a single point $q$.

Choose a nowhere zero smooth vector field $V$ on $D$ and let $\hat{V}:=\frac{V}{\|V\|}$ the induced unit vector field on $D \cap \dot{S}$. Let $\eta$ be a path contained in a coordinate chart near $q$ and that simply winds around $q$. Clearly, in such a coordinate chart $\left.V\right|_{\eta}$ and so $\left.\hat{V}\right|_{\eta}$ have winding number \pm 2 . Finally, choose a point $\bar{p} \in \mathbb{S}^{2}$ and a $\bar{v} \in T_{\bar{p}}^{1} \mathbb{S}^{2}$.

Represent an element in $\pi_{1}(\dot{S}, p)$ as a path $\gamma:[0,1] \rightarrow \dot{S} \cap D$. By Lemma 2.2(ii) and Lemma 2.1(iii), there exists a unique developing map $\operatorname{dev}_{\gamma}$ that takes $(p, \hat{V}(p)) \in T_{S}^{1}$ to $(\bar{p}, \bar{v}) \in T^{1} \mathbb{S}^{2}$. For every $t \in[0,1]$ let $R_{\gamma}(t) \in \mathrm{SO}(3, \mathbb{R})$ be the unique transformation that takes $d\left(\operatorname{dev}_{\gamma}\right)_{0}(V(\gamma(0)))=$ $(\bar{p}, \bar{v})$ to $d\left(\operatorname{dev}_{\gamma}\right)_{t}(V(\gamma(t)))$. The path $R_{\gamma}:[0,1] \rightarrow \mathrm{SO}(3, \mathbb{R})$ is clearly continuous and satisfies $R_{\gamma}(0)=I$ and $R_{\gamma}(1)=\rho(\gamma)$.

Let $\hat{R}_{\gamma}:[0,1] \rightarrow \mathrm{SU}(2)$ be the unique continuous lift of $R_{\gamma}$ via the standard double cover $\mathrm{SU}(2) \rightarrow \mathrm{SO}(3, \mathbb{R})$ such that $\hat{R}_{\gamma}(0)=I$. Define $\hat{\rho}(\gamma):=\hat{R}_{\gamma}(1)$.

If $s \mapsto \gamma_{s}$ is a continuous family of loops in $\dot{S} \cap D$ based at $p$, then $\rho\left(\gamma_{s}\right)=\rho\left(\gamma_{0}\right)$ and so $\hat{\rho}\left(\gamma_{s}\right)=\hat{\rho}\left(\gamma_{0}\right)$ by continuity. Thus, two loops based at $p$ that are homotopic in $\dot{S} \cap D=\dot{S} \backslash\{q\}$ have the same $\mathrm{SU}(2)$-holonomy: this defines a representation $\pi_{1}(\dot{S} \backslash\{q\}, p) \rightarrow \mathrm{SU}(2)$.

In order to see that the constructed $\mathrm{SU}(2)$-representation descends to $\pi_{1}(\dot{S}, p) \cong \pi_{1}(\dot{S} \backslash\{q\}, p) /\langle\eta\rangle$, it is enough to check that the $\mathrm{SU}(2)$-holonomy along $\eta$ is trivial. As $\left.\hat{V}\right|_{\eta}$ winds twice, $q$ is a smooth 
point for the metric of $S$ and $\eta$ is freely homotopic to $\gamma_{1} * \cdots * \gamma_{n}$, we obtain $\hat{\rho}\left(\gamma_{1}\right) \cdots \hat{\rho}\left(\gamma_{n}\right)=$ $\hat{\rho}(\eta)=I$.

Hence, we conclude that $\hat{\rho}: \pi_{1}(\dot{S}, p) \rightarrow \mathrm{SU}(2)$ is a well-defined representation that lifts $\rho$.

Step 2: eigenvalues of $\hat{\rho}\left(\gamma_{j}\right)$.

Let $p^{\prime}$ be a point very close to $x_{j}$ and let $\beta$ be a loop based at $p^{\prime}$ that keeps at constant distance from $x_{j}$ and that simply winds around $x_{j}$ at constant speed. Clearly, the path $\gamma_{j}$ is homotopic to $\gamma_{j}^{\prime}=\alpha^{-1} * \beta * \alpha$, where $\alpha$ is a suitable simple path from $p$ to a point $p^{\prime}$.

Thus, $R_{\gamma_{j}^{\prime}}(1)$ can be written as $A^{-1} B A$, where $A=R_{\gamma_{j}^{\prime}}\left(\frac{1}{3}\right)$ and $B=R_{\gamma_{j}^{\prime}}\left(\frac{2}{3}\right) R_{\gamma_{j}^{\prime}}\left(\frac{1}{3}\right)^{-1}$, and so $\hat{R}_{\gamma_{j}^{\prime}}(1)=\hat{A}^{-1} \hat{B} \hat{A}$ for $\hat{A}=\hat{R}_{\gamma_{j}^{\prime}}\left(\frac{1}{3}\right)$ and $\hat{B}=\hat{R}_{\gamma_{j}^{\prime}}\left(\frac{2}{3}\right) \hat{R}_{\gamma_{j}^{\prime}}\left(\frac{1}{3}\right)^{-1}$. By our choice of $\beta$, the path $[0,1] \ni t \mapsto R_{\gamma_{j}^{\prime}}\left(\frac{1+t}{3}\right) R_{\gamma_{j}^{\prime}}\left(\frac{1}{3}\right)^{-1}$ is very close to be a rotation about a fixed axis of constant speed $2 \pi \vartheta_{j}$ and $B$ is a rotation of angle $2 \pi \vartheta_{j}$. As a consequence, $[0,1] \ni t \mapsto \hat{R}_{\gamma_{j}^{\prime}}\left(\frac{1+t}{3}\right) \hat{R}_{\gamma_{j}^{\prime}}\left(\frac{1}{3}\right)^{-1}$ is very close to be conjugate to the path $[0,1] \ni t \mapsto \operatorname{diag}\left(e^{i t \pi\left(\vartheta_{j}-1\right)}, e^{-i t \pi\left(\vartheta_{j}-1\right)}\right)$. Thus, $\hat{B}$ has eigenvalues $e^{ \pm i \pi\left(\vartheta_{j}-1\right)}$ and so the same holds for $\hat{\rho}\left(\gamma_{j}\right)$.

Step 3: the lift $\hat{\rho}$ is canonical.

Consider first another nowhere vanishing vector field $W$ on $D$ and let $\hat{W}=\frac{W}{\|W\|}$. There exists a continuous function $\bar{a}: D \rightarrow \mathbb{R} / \mathbb{Z}$ such that $\hat{W}(x)$ is obtained from $\hat{V}(x)$ by a counterclockwise rotation of an angle $2 \pi \bar{a}(x)$. Since $D$ is simply connected, the function $\bar{a}$ lifts to a continuous $a: D \rightarrow \mathbb{R}$. If we call $\hat{V}_{s}(x)$ the vector obtained by rotating $\hat{V}(x)$ counteclockwise by $s \cdot 2 \pi a(x)$ for all $s \in[0,1]$, then $s \mapsto \hat{V}_{s}$ is a continuous family of unit vector fields on $D$ with $\hat{V}_{0}=\hat{V}$ and $\hat{V}_{1}=\hat{W}$. This induces a continuous family $s \mapsto \hat{\rho}_{s}$ of lifts of $\rho$, which must thus be the constant family. Hence, $\hat{\rho}_{0}=\hat{\rho}_{1}$ and so $\hat{\rho}$ does not depend on the choice of the vector field.

Finally, if $D^{\prime}=S \backslash\left\{q^{\prime}\right\}$ is another disk, then there is an isotopy that moves $q$ to $q^{\prime}$ fixing $\left\{x_{1}, \ldots, x_{n}\right\}$ and so it moves $\dot{S} \cap D$ to $\dot{S} \cap D^{\prime}$. Again, this determines a continuous family of lifts of $\rho$, which must then be constantly equal to $\hat{\rho}$.

We remark that the coaxiality condition for the $\mathrm{SO}(3, \mathbb{R})$-valued holonomy representation can be rephrased in a more familiar way in terms of its lift.

Lemma 2.17 (Non-coaxial subgroups). Let $\hat{G}$ be a subgroup of $\mathrm{SU}(2)$ and let $G$ be its image via the natural projection $\mathrm{SU}(2) \rightarrow \mathrm{SO}(3, \mathbb{R})$.

(a) The group $\hat{G}$ is commutative if and only if $G$ belongs to a 1-parameter subgroup of $\mathrm{SO}(3, \mathbb{R})$. Hence, the canonical lift $\hat{\rho}$ is Abelian $\Longleftrightarrow$ the representation $\rho$ is coaxial.

(b) If $G$ is non-coaxial and $\tau \in \operatorname{PSL}(2, \mathbb{C})$ commutes with all elements in $G$, then $\tau \in \operatorname{SO}(3, \mathbb{R})$. Hence, if $\tau \rho \tau^{-1}=\rho$ and $\rho$ is non-coaxial, then $\tau \in \operatorname{SO}(3, \mathbb{R})$.

Proof. Since unitary matrices are diagonalizable, then $\hat{G}$ is Abelian if and only if all matrices in $\hat{G}$ are simultaneously diagonalizable. This occurs if and only if $\hat{G}$ is contained in a 1-parameter subgroup of $\mathrm{SU}(2)$, which is equivalent to asking that $G$ is contained in a 1-parameter subgroup of $\operatorname{SO}(3, \mathbb{R})$. This proves (a).

As for (b), let $\hat{\tau} \in \operatorname{SL}(2, \mathbb{C})$ be a lift of $\tau$, so that $\hat{\tau} \hat{\gamma}= \pm \hat{\gamma} \hat{\tau}$ for every $\hat{\gamma} \in \hat{G}$. Up to conjugation by a matrix in $\mathrm{SU}(2)$, we can assume that

$$
\hat{\tau}=\left(\begin{array}{cc}
\lambda & z \\
0 & \lambda^{-1}
\end{array}\right)
$$

with $\lambda, z \in \mathbb{C}$ and $|\lambda| \geq 1$, so that

$$
h:=\overline{\hat{\tau}}^{T} \hat{\tau}=\left(\begin{array}{cc}
|\lambda|^{2}+|z|^{2} & z \bar{\lambda}^{-1} \\
\bar{z} \lambda^{-1} & |\lambda|^{-2}
\end{array}\right)
$$

has $\operatorname{det}(h)=1$ and $t:=\frac{1}{2} \operatorname{tr}(h)=\frac{1}{2}\left(|\lambda|^{2}+|\lambda|^{-2}+|z|^{2}\right) \geq 1$. Thus, $h$ is diagonalizable and it has eigenvalues $\mu^{2}$ and $\mu^{-2}$, with $\mu=\sqrt{t+\sqrt{t^{2}-1}} \geq 1$. It is easy to see that $\|\tau v\| \leq \mu\|v\|$ for every $v \in \mathbb{C}^{2}$ and equality holds if and only if $v$ belongs to the $\mu^{2}$-eigenspace $E_{\mu^{2}} \subseteq \mathbb{C}^{2}$ of $h$. Since $\|\tau(\hat{\gamma}(v))\|=\|\hat{\gamma}(\tau(v))\|=\|\tau(v)\| \leq \mu\|v\|=\mu\|\hat{\gamma}(v)\|$, every $\hat{\gamma} \in \hat{G}$ preserves $E_{\mu^{2}}$. 
By (a), the group $\hat{G}$ is not Abelian and so $E_{\mu^{2}}$ cannot be 1-dimensional. This implies that $t=1$ and so $|\lambda|=1$ and $z=0$, which shows that $\hat{\tau} \in \mathrm{SU}(2)$ and finally $\tau \in \mathrm{SO}(3, \mathbb{R})$.

\subsubsection{Matrices in $\mathrm{SU}(2)$ and broken geodesics on $\mathbb{S}^{3}$}

In view of Corollary 2.16] it is natural first to discuss the following.

Problem 2.18. Find a criterion for the existence of a standard set of matrices $U_{1}, \ldots, U_{n} \in \mathrm{SU}(2)$ for $\vartheta \in \mathbb{R}_{+}^{n}$ that do not simultaneusly commute.

This problem was addressed in many papers and explicit inequalities are known (see [2]). In order to motivate these inequalities, we recall how this question is equivalent to a different question about broken geodesics on the standard 3-sphere $\mathbb{S}^{3}$.

Notation. By broken geodesic on $\mathbb{S}^{3}$ we will mean a piecewise geodesic path with endpoints $v_{0}$ and $v_{n}$ that passes through an ordered collection of points $v_{0}, \ldots, v_{n}$ of $\mathbb{S}^{3}$ in such a way that each side $s_{j}$ going from the vertex $v_{j-1}$ to the vertex $v_{j}$ is of minimal length (and so at most $\pi$ ).

Given a broken geodesic on $\mathbb{S}^{3}$ with vertices $v_{0}, \ldots, v_{n}$, we define $U_{j} \in \mathrm{SU}(2)$ as the unique transformation that takes $v_{j-1}$ to $v_{j}$ for $j=1, \ldots, n$.

Vice versa, given matrices $U_{1}, \ldots, U_{n}$ in $\mathrm{SU}(2)$ and fixed a basepoint $v_{0}:=(1,0)$ on the unit sphere $\mathbb{S}^{3} \subset \mathbb{C}^{2}$, we define $v_{j}:=U_{j}\left(v_{j-1}\right)=U_{j} U_{j-1} \cdots U_{1}\left(v_{0}\right)$ for $j=1, \ldots, n$. A broken geodesic $\Gamma$ is then obtained by drawing a shortest geodesic $s_{j}$ from $v_{j-1}$ to $v_{j}$ for all $j=1, \ldots, n$. Notice that, given $v_{j-1}$, the segment $s_{j}$ is uniquely determined unless $U_{j}=-I$.

Clearly, the matrices $U_{j}$ satisfy $U_{1} \cdots U_{n}=I$ if and only if $v_{n}=v_{0}$, i.e. if and only if the broken geodesic is closed.

Definition 2.19. Let $\boldsymbol{\vartheta} \in \mathbb{R}^{n}$ be an angle vector. Its associated defect vector is $\boldsymbol{\delta}:=\boldsymbol{\vartheta}-\mathbf{1} \in \mathbb{R}^{n}$, where $\mathbf{1}=(1,1, \ldots, 1)$. The associated reduced angle vector $\overline{\boldsymbol{\vartheta}} \in \mathbb{R}^{n}$ is defined in such a way that $\bar{\vartheta}_{j} \in[0,2)$ and $\vartheta_{j}-\bar{\vartheta}_{j} \in 2 \mathbb{Z}$. Finally, the reduced defect vector is $\overline{\boldsymbol{\delta}}:=\overline{\boldsymbol{\vartheta}}-\mathbf{1} \in[-1,1)^{n}$.

Remark 2.20. The definition of $\overline{\boldsymbol{\vartheta}}$ is motivated by the fact that the edge $s_{j}$ of the broken geodesic on $\mathbb{S}^{3}$ associated to $U_{1}, \ldots, U_{n}$ has length $\ell_{j}=\pi\left|1-\bar{\vartheta}_{j}\right|=\pi\left|\bar{\delta}_{j}\right|$.

We summarize the content of the above discussion into the following.

Lemma 2.21 (Broken geodesics and standard set of matrices). Let $\vartheta_{1}, \ldots, \vartheta_{n}>0$. Then the following are equivalent:

(a) there exists a closed broken geodesic on $\mathbb{S}^{3}$ with $n$ edges of length $\ell_{j}=\pi\left|\bar{\delta}_{j}\right|$ for $j=1, \ldots, n$;

(b) there exists a standard sets of matrices $U_{1}, \ldots, U_{n} \in \mathrm{SU}(2)$ for $\vartheta$.

Notice that, through the identification $\mathrm{SU}(2) \ni U \mapsto U\left(v_{0}\right) \in \mathbb{S}^{3}$, there is a correspondence between 1-parameters subgroups of $\mathrm{SU}(2)$ and maximal circles on $\mathbb{S}^{3}$ through $v_{0}$. Thus, the matrices $U_{1}, \ldots, U_{n}$ simultaneously commute if and only if $v_{0}, \ldots, v_{n}$ all belong to the same maximal circle.

The following result is essentially contained in [2].

Theorem 2.22 (Constraints for broken geodesics). There exists a closed broken geodesic on $\mathbb{S}^{3}$ with $n$ edges of length $\ell_{1}, \ldots, \ell_{n} \in[0, \pi]$ if and only if

$$
\sum_{j \in X}\left(\pi-\ell_{j}\right)+\sum_{k \in X^{c}} \ell_{k} \geq \pi
$$

for all $X \subseteq\{1, \ldots, n\}$ with $|X|$ odd.

Remark 2.23. These inequalities are generalizations of the following simple statement: there cannot be a closed broken geodesic on $\mathbb{S}^{3}$ with odd number of edges of length $\pi$. Moreover, even if we replace each length $\ell_{j}=\pi$ by $\ell_{j}=\pi \pm \varepsilon_{j}$ with $\sum_{j}\left|\varepsilon_{j}\right|<\pi$, then a closed broken geodesic cannot exist.

Lemma 2.24 (Broken geodesics on a maximal circle). Equality in (Pol) has the following geometric counterpart. 
(i) If equality is attained in ( $\mathrm{Pol}$ ) for a certain $X$, then every closed broken geodesic on $\mathbb{S}^{3}$ with edges of lengths $\ell_{j}$ sits on a maximal circle.

(ii) If a closed broken geodesic on $\mathbb{S}^{3}$ with edges of lengths $\ell_{j}$ sits on a maximal circle, then

$$
\sum_{j \in Y} \ell_{j}-\sum_{k \in Y^{c}} \ell_{k} \equiv 0 \quad(\bmod 2 \pi)
$$

for some subset $Y \subseteq\{1, \ldots, n\}$.

Proof. Indeed, (ii) is immediate: once fixed an orientation on the maximal circle, just let $Y$ be the collection of positively oriented edges of the broken geodesic. About (i), it is enough to notice that, if a broken geodesic $\Gamma$ does not sit on a maximal circle, then it can be deformed in such a way that the quantity on the left-hand side of ( $\mathrm{Pol})$ decreases.

The above characterization of the lengths of the edges of closed broken geodesics on $\mathbb{S}^{3}$ gives a criterion for the existence of matrices that satisfy the conditions of Problem 2.18. Now we want to rewrite such criterion in a more compact way.

Corollary 2.25 (Angle constraints for representations in SU(2)). Given $\vartheta_{1}, \ldots, \vartheta_{n}>0$, the following facts are equivalent.

(1) There exists a standard set of matrices $U_{1}, \ldots, U_{n} \in \mathrm{SU}(2)$ for $\boldsymbol{\vartheta}$.

(2) The following inequalities hold

$$
\sum_{j \in X}\left(1-\left|\bar{\delta}_{j}\right|\right)+\sum_{k \in X^{c}}\left|\bar{\delta}_{k}\right| \geq 1
$$

for all $X \subseteq\{1, \ldots, n\}$ with $|X|$ odd.

(3) The following inequality holds

$$
d_{1}\left(\boldsymbol{\delta}, \mathbb{Z}_{o}^{n}\right) \geq 1
$$

Also, equality holds in (3) if and only if it holds in (2) for a certain X. Moreover:

(I) if $d_{1}\left(\boldsymbol{\delta}, \mathbb{Z}_{o}^{n}\right)=1$, then all standard $n$-uples of matrices $U_{1}, \ldots, U_{n}$ for $\boldsymbol{\vartheta}$ simultaneously commute and in fact they belong to the same 1-parameter subgroup of $\mathrm{SU}(2)$;

(II) if there exists a standard $n$-uple of matrices $U_{1}, \ldots, U_{n}$ that belong to the same 1-parameter subgroup of $\mathrm{SU}(2)$, then

$$
\sum_{j \in Y} \vartheta_{j}-\sum_{k \in Y^{c}} \vartheta_{k} \equiv 0 \quad(\bmod 2)
$$

for a certain subset $Y \subseteq\{1, \ldots, n\}$.

Proof. The equivalence (1) $\Longleftrightarrow(2)$ is just a rephrasing of Theorem 2.22. Moreover, it is clear that (I) and (II) are rephrasings of (i) and (ii) in Lemma 2.24 So it is enough to show that $(2) \Longleftrightarrow(3)$, which is a consequence of the following equality

$$
d_{1}\left(\boldsymbol{\delta}, \mathbb{Z}_{o}^{n}\right)=\inf _{|X| \text { odd }}\left(\sum_{j \in X}\left(1-\left|\bar{\delta}_{j}\right|\right)+\sum_{k \in X^{c}}\left|\bar{\delta}_{k}\right|\right)
$$

Let $\boldsymbol{m} \in \mathbb{Z}_{o}^{n}$ and call $X$ the subset of indices in $\{1, \ldots, n\}$ for which $m_{i}$ is odd. Clearly, $|X|$ is odd because $\|\boldsymbol{m}\|_{1}$ is. It is easy to see that $\left|\delta_{j}-m_{j}\right| \geq 1-\left|\bar{\delta}_{j}\right|$ for $j \in X$, and $\left|\delta_{k}-m_{k}\right| \geq\left|\bar{\delta}_{k}\right|$ for $k \in X^{c}$. Thus, $d_{1}(\boldsymbol{\delta}, \boldsymbol{m}) \geq \sum_{j \in X}\left(1-\left|\bar{\delta}_{j}\right|\right)+\sum_{k \in X^{c}}\left|\bar{\delta}_{k}\right|$. Moreover, the equality is attained for those $\boldsymbol{m} \in \mathbb{Z}_{o}^{n}$ for which $\left|\delta_{j}-m_{j}\right| \leq 1$ for all $j=1, \ldots, n$. Thus, equation ( $\left.\mathrm{H=Pol}\right)$ holds.

As a consequence, we can determine a necessary condition for the existence of a metric of curvature 1 on $\mathbb{S}^{2}$ with cone points of angles $\vartheta_{1}, \ldots, \vartheta_{n}$.

Proof of Theorem $A$, It follows by combining Corollary 2.16 and implication (1) $\Longrightarrow$ (3) in Corollary 2.25 


\subsection{Algebraic merging}

The main goal of this section is to prove Theorem $\mathrm{D}$ In order to do that, we first need to set some notation.

Given $\boldsymbol{\vartheta}=\left(\vartheta_{1}, \ldots, \vartheta_{n}\right) \in \mathbb{R}_{+}^{n}$, we recall that the defect vector is $\boldsymbol{\delta}=\boldsymbol{\vartheta}-\mathbf{1}$. Throughout this section it will turn more practical to directly work with $\boldsymbol{\delta}$ instead of $\boldsymbol{\vartheta}$.

Notation. Denote by $\mathcal{H}^{n}$ the subset of $\boldsymbol{\delta} \in \mathbb{R}^{n}$ that satisfy the holonomy constraints, namely such that $d_{1}\left(\boldsymbol{\delta}, \mathbb{Z}_{o}^{n}\right) \geq 1$. This is the complement in $\mathbb{R}^{n}$ of a union of octahedrons centred at points of $\mathbb{Z}_{o}^{n}$. Denote by $\mathcal{P}^{n}$ the subset of $\boldsymbol{\delta} \in \mathbb{R}^{n}$ that satisfy the positivity constraints, namely such that $\delta_{1}+\cdots+\delta_{n}>-2$ and $\delta_{1}, \ldots, \delta_{n}>-1$. The locus $\mathcal{P}^{n} \cap \mathcal{H}^{n}$ of admissible defect vectors will be denoted by $\mathcal{A}^{n}$.

Let $n \geq 4$ and let $i, j \in\{1, \ldots, n\}$ be distinct. We define the positive/negative algebraic merging operation

$$
M_{(i \pm j)}: \mathbb{R}^{n} \longrightarrow \mathbb{R}^{n-1}
$$

as $M_{(i+j)}\left(\delta_{1}, \ldots, \delta_{n}\right):=\left(\delta_{1}, \ldots, \widehat{\delta}_{i}, \ldots, \widehat{\delta}_{j}, \ldots, \delta_{n}, \delta_{i}+\delta_{j}\right)$ and $M_{(i-j)}\left(\delta_{1}, \ldots, \delta_{n}\right):=\left(\delta_{1}, \ldots, \widehat{\delta}_{i}, \ldots, \widehat{\delta}_{j}, \ldots, \delta_{n}, \delta_{i}-\right.$ $\left.\delta_{j}-2\right)$.

Lemma 2.26 (Basic properties of $\left.M_{(i \pm j)}\right)$. Every algebraic merging operation $M: \mathbb{R}^{n} \rightarrow \mathbb{R}^{n-1}$ satisfies the following properties:

(a) $M\left(\mathbb{Z}_{o}^{n}\right)=\mathbb{Z}_{o}^{n-1}$;

(b) $M$ is contracting for the $\ell^{1}$ metrics;

(c) $M(\boldsymbol{\delta}) \in \operatorname{int}\left(\mathcal{H}^{n-1}\right) \Longrightarrow \delta \in \operatorname{int}\left(\mathcal{H}^{n}\right)$.

Proof. Property (a) is obvious and claim (c) follows from (a) and (b). As for (b), up to reordering the coordinates we can assume that $M=M_{(1+2)}$ or $M=M_{(1-2)}$.

Let $\boldsymbol{m}, \boldsymbol{m}^{\prime} \in \mathbb{R}^{n}$. Then

$$
\begin{aligned}
d_{1}\left(M_{(1+2)}(\boldsymbol{m}), M_{(1+2)}\left(\boldsymbol{m}^{\prime}\right)\right) & =\left|\left(m_{1}+m_{2}\right)-\left(m_{1}^{\prime}+m_{2}^{\prime}\right)\right|+\sum_{j=3}^{n}\left|m_{j}-m_{j}^{\prime}\right| \leq \\
& \leq\left|m_{1}-m_{1}^{\prime}\right|+\left|m_{2}-m_{2}^{\prime}\right|+\sum_{j=3}^{n}\left|m_{j}-m_{j}^{\prime}\right|=d_{1}\left(\boldsymbol{m}, \boldsymbol{m}^{\prime}\right) .
\end{aligned}
$$

The proof for $M=M_{(1-2)}$ is completely analogous.

The main result of this section is the following more precise version of Theorem D,

Theorem 2.27 (Algebraic merging). Let $n \geq 5$ and suppose that $\boldsymbol{\delta} \in \operatorname{int}\left(\mathcal{A}^{n}\right)$. Then there exist distinct indices $i, j \in\{1, \ldots, n\}$ such that at least one of the following holds:

(a) $M_{(i+j)}(\boldsymbol{\delta}) \in \operatorname{int}\left(\mathcal{A}^{n-1}\right)$;

(b) $M_{(i-j)}(\boldsymbol{\delta}) \in \operatorname{int}\left(\mathcal{A}^{n-1}\right)$ and $\delta_{i}, \delta_{j}, \delta_{i}-\delta_{j} \notin \mathbb{Z}$.

In order to prove the above result, we will separately analyze three different cases. We will see that in most situations it is possible to find indices $i, j$ such that (a) holds.

\subsubsection{Intersection of $\mathcal{A}^{n}$ with a unit integer cube}

Throughout this section, we assume $n \geq 3$.

Notation. We will use the symbol $\square^{n}$ to denote any closed unit cube with integer vertices in $\mathbb{R}^{n}$ and the symbol $\diamond^{n}$ to denote the truncated cube obtained by intersecting $\square^{n}$ with $\mathcal{H}^{n}$. Sometimes, we will use the notation $\square_{c}$ to indicate the unit cube with center in $\boldsymbol{c}=\left(c_{1}, \ldots, c_{n}\right) \in \mathbb{R}^{n}$. 


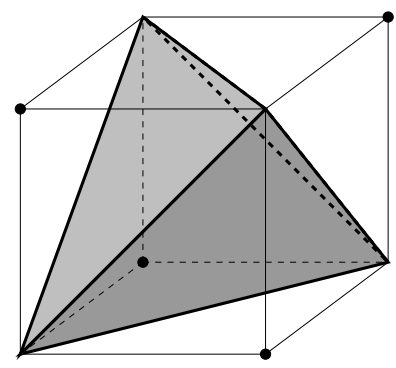

- Odd-integral

vertices of the cube

Figure 1: A 3-dimensional truncated cube $\diamond^{3}$.

Lemma 2.28 (Truncated cubes). Let $\square^{n}$ be a unit cube in $\mathbb{R}^{n}$ with integer vertices. The intersection $\diamond^{n}=\square^{n} \cap \mathcal{H}^{n}$ is the convex hull of all even vertices of $\square^{n}$.

Proof. Note that $\square^{n} \cap \mathcal{H}^{n}$ consist of all points of $\square^{n}$ that are on $\ell^{1}$ distance at least one from all odd vertices of $\square^{n}$. If $\boldsymbol{m}$ is such an odd vertex, then the points in $\square^{n}$ at distance at most 1 from $\boldsymbol{m}$ are formed by the simplex spanned by $\boldsymbol{m}$ and the $n$ even vertices of $\square^{n}$ at distance 1 from $\boldsymbol{m}$. Hence, the set $\square^{n} \cap \mathcal{H}^{n}$ is obtained from $\square^{n}$ by cutting away $2^{n-1}$ simplices corresponding to the odd vertices of $\square^{n}$. It follows that $\diamond^{n}$ is a convex polytope and it is easy to see that its vertices are the even vertices of $\square^{n}$

Remark 2.29. Since $\mathcal{P}^{n}$ is convex and $\mathcal{A}^{n}=\mathcal{P}^{n} \cap \mathcal{H}^{n}$ it follows from the lemma that the intersection of any integral unit cube $\square^{n}$ with $\mathcal{A}^{n}$ is convex.

As a consequence, we deduce the connectedness of $\mathcal{H}^{n}$ and of $\mathcal{A}^{n}$ for $n \geq 4$.

Proof of Lemma $B$. Each $n$-dimensional simplex corresponding to an even vertex of $\square^{n}$ has volume $1 / n$ ! and so $\diamond^{n}$ has volume $1-\frac{2^{n-1}}{n !}>0$, because $n \geq 3$. Hence, all $\diamond^{n}$ have non-empty interior and so, in particular, $\mathcal{H}^{n}$ and $\mathcal{A}^{n}$ are non-empty.

Let now $n \geq 4$. We claim that, if $\boldsymbol{c}=\left(c_{1}, \ldots, c_{n}\right)$ is the center of a unit cube $\square_{\boldsymbol{c}}$ and $\boldsymbol{e}_{\boldsymbol{i}}$ is a vector in the standard basis of $\mathbb{R}^{n}$, then the interior of $\diamond_{c} \cup \diamond_{c+e_{i}}$ is connected. In fact, the two adjacent truncated cubes $\nabla_{\boldsymbol{c}}$ and $\nabla_{\boldsymbol{c}+\boldsymbol{e}_{\boldsymbol{i}}}$ share a face $\mathcal{F}$ isometric to a lower dimensional truncated cube $\diamond_{\boldsymbol{c}^{\prime}}$, where $\boldsymbol{c}^{\prime}=\left(c_{1}, \ldots, \widehat{c}_{i}, \ldots, c_{n}\right)$. As $n-1 \geq 3$, the $\operatorname{interior} \operatorname{int}(\mathcal{F}) \cong \operatorname{int}\left(\diamond_{\boldsymbol{c}^{\prime}}\right)$ (as a subset of $\left.\mathbb{R}^{n-1}\right)$ is non-empty: let $\boldsymbol{\delta}$ be a point in $\operatorname{int}(\mathcal{F})$. As $\operatorname{int}\left(\diamond_{\boldsymbol{c}} \cup \diamond_{\boldsymbol{c}+\boldsymbol{e}_{\boldsymbol{i}}}\right)$ is star-shaped with respect to $\boldsymbol{\delta}$, the claim follows.

One then easily concludes that $\operatorname{int}\left(\mathcal{H}^{n}\right)$ and $\operatorname{int}\left(\mathcal{A}^{n}\right)$ are connected for $n \geq 4$.

Note that the boundary $\partial \diamond^{n}$ of a truncated $n$-cube is made of $2^{n-1}$ faces isometric to $(n-1)$ simplices (one for each odd vertex of $\square^{n}$ ) and $2 n$ faces isometric to truncated $(n-1)$ )-cubes (one for each face of $\square^{n}$ ). All faces have an interior part for $n \geq 4$ (see Figure 2), whereas the non-simplicial faces of $2 \diamond^{3}$ are degenerate, as it appears clearly in Figure 1.

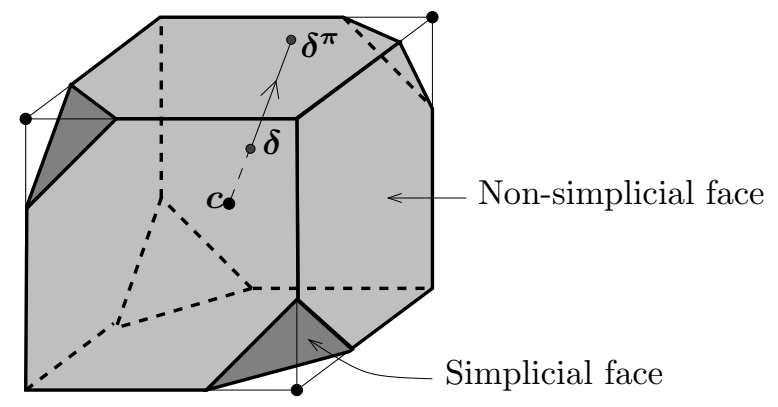

Figure 2: Symbolic picture of a truncated cube $\diamond^{n}$ with $n \geq 4$.

Notation. Denote by $\boldsymbol{c}$ the center of $\square^{n}$, which is a point with half-integral coefficients. For any $\delta \in \diamond^{n}$ different from $\boldsymbol{c}$ denote by $\boldsymbol{\delta}^{\pi}$ the projection of $\boldsymbol{\delta}$ to the boundary of $\diamond^{n}$ from the center $\boldsymbol{c}$, i.e. the unique point on $\partial \diamond^{n}$ such that $\boldsymbol{\delta}$ belongs to the segment that joins $\boldsymbol{\delta}^{\pi}$ and $\boldsymbol{c}$. 
In what follows we will distinguish two types of points in $\diamond^{n}$.

Definition 2.30. A point $\delta \in \diamond^{n}$ different from its center is called simplicial if $\boldsymbol{\delta}^{\pi}$ belongs to a simplicial face of $\partial \diamond^{n}$; otherwise, $\boldsymbol{\delta}$ is called non-simplicial.

The following lemma summarizes some simple useful properties of simplicial and non-simplicial points.

Lemma 2.31 (Boundary of truncated cubes). Let $\boldsymbol{\delta}$ be a point in a truncated cube $\diamond^{n}$ different from its center $\boldsymbol{c}$. Then the following hold.

(a) If $\boldsymbol{\delta}$ is non-simplicial, then $d_{1}\left(\boldsymbol{\delta}^{\pi}, \mathbb{Z}_{o}^{n}\right)>1$ and there exists an $i$ such that $\delta_{i}^{\pi} \in \mathbb{Z}$.

(b) $\boldsymbol{\delta}$ is simplicial if and only if $d_{1}\left(\boldsymbol{\delta}^{\pi}, \mathbb{Z}_{o}^{n}\right)=1$.

(c) Suppose that $\boldsymbol{\delta}$ is a simplicial point and let $\boldsymbol{m}$ be a corresponding odd-integral vertex of $\square^{n}$. Then $\boldsymbol{m}$ is a closest point to $\boldsymbol{\delta}$ among all odd-integral points.

Proof. The first two statements directly follow from the definitions, so we only prove (c). Let $\boldsymbol{m}^{(\mathbf{1})}, \ldots, \boldsymbol{m}^{(\boldsymbol{n})}$ be the even vertices of $\square^{n}$ sitting at $\ell^{1}$ distance 1 from $\boldsymbol{m}$. Since $\boldsymbol{\delta}$ is simplicial it lies in the convex hull $\mathcal{K}$ of points $\boldsymbol{c}, \boldsymbol{m}^{(\mathbf{1})}, \ldots, \boldsymbol{m}^{(\boldsymbol{n})}$.

Let $\boldsymbol{m}^{\prime}$ be any other point in $\mathbb{Z}_{o}^{n}$ and denote $\boldsymbol{\delta}^{\prime}$ a point in $\mathcal{K}$. Note that both functions $f=d_{1}(\bullet, \boldsymbol{m})$ and $f^{\prime}=d_{1}\left(\bullet, \boldsymbol{m}^{\prime}\right)$ are affine on $\mathcal{K}$. So in order to prove that $d_{1}(\boldsymbol{\delta}, \boldsymbol{m}) \leq d_{1}\left(\boldsymbol{\delta}, \boldsymbol{m}^{\prime}\right)$ it is enough to show that $f(\boldsymbol{p}) \leq f^{\prime}(\boldsymbol{p})$ for every vertex $\boldsymbol{p}$ of $\mathcal{K}$. This is indeed so, because $d_{1}\left(\boldsymbol{m}^{\prime}, \boldsymbol{c}\right) \geq d_{1}(\boldsymbol{m}, \boldsymbol{c})=\frac{n}{2}$ and $d_{1}\left(\boldsymbol{m}^{\prime}, \boldsymbol{m}^{(i)}\right) \geq d_{1}\left(\boldsymbol{m}, \boldsymbol{m}^{(i)}\right)=1$.

\subsubsection{Simplicial and non-simplicial merging}

Even if we begin with an admissible defect vector $\boldsymbol{\delta}$, the output of a positive algebraic merging operation might be no longer admissible. As positivity issues are generally easier to keep under control, here we focus on the problem of determining whether $M_{(i+j)}(\boldsymbol{\delta})$ belongs to int $\left(\mathcal{H}^{n-1}\right)$ for given $i \neq j$ and $\boldsymbol{\delta} \in \operatorname{int}\left(\mathcal{H}^{n}\right)$.

First, a simple observation about merging integral angles.

Lemma 2.32 (Integral merging). Let $\boldsymbol{\delta} \in \mathbb{R}^{n}$ be a vector such that $\delta_{i} \in \mathbb{Z}$ for some $i$. Let $M$ be a merging operation of type $M_{(i+j)}, M_{(i-j)}, M_{(j-i)}$ for some $j \neq i$. Then $d_{1}\left(M(\boldsymbol{\delta}), \mathbb{Z}_{o}^{n-1}\right)=d_{1}\left(\boldsymbol{\delta}, \mathbb{Z}_{o}^{n}\right)$. Hence, $M(\boldsymbol{\delta}) \in \operatorname{int}\left(\mathcal{H}^{n-1}\right)$ if and only if $\boldsymbol{\delta} \in \operatorname{int}\left(\mathcal{H}^{n}\right)$.

Proof. Since $M\left(\mathbb{Z}_{o}^{n}\right) \subseteq \mathbb{Z}_{o}^{n-1}$ and $M$ is contracting for the $\ell^{1}$ distances, we have $d_{1}\left(M(\boldsymbol{\delta}), \mathbb{Z}_{o}^{n-1}\right) \leq$ $d_{1}\left(\boldsymbol{\delta}, \mathbb{Z}_{o}^{n}\right)$. It is then enough to show that $d_{1}\left(\boldsymbol{\delta}, \mathbb{Z}_{o}^{n}\right) \leq d_{1}\left(M(\boldsymbol{\delta}), \mathbb{Z}_{o}^{n-1}\right)$. We will prove it for $M=M_{(i+j)}$, the other cases being analogous. Moreover, up to reordering the coordinates, we can assume that $i=1$ and $j=2$.

For every $\boldsymbol{m} \in \mathbb{Z}_{o}^{n-1}$, we define $\boldsymbol{m}^{\prime}:=\left(\delta_{1}, m_{n-1}-\delta_{1}, m_{1}, m_{2}, \ldots, m_{n-2}\right) \in \mathbb{Z}_{o}^{n}$ so that $M_{(1+2)}\left(\boldsymbol{m}^{\prime}\right)=\boldsymbol{m}$. Now, $d_{1}\left(\boldsymbol{\delta}, \boldsymbol{m}^{\prime}\right)=\left|\delta_{1}-\delta_{1}\right|+\left|\delta_{2}-\left(m_{n-1}-\delta_{1}\right)\right|+\sum_{j=3}^{n}\left|\delta_{j}-m_{j-2}\right|=$ $d_{1}\left(M_{(1+2)}(\boldsymbol{\delta}), \boldsymbol{m}\right)$, since $M_{(1+2)}(\boldsymbol{\delta})=\left(\delta_{3}, \ldots, \delta_{n}, \delta_{1}+\delta_{2}\right)$. This shows that $d_{1}\left(\boldsymbol{\delta}, \mathbb{Z}_{o}^{n}\right) \leq d_{1}\left(M_{(1+2)}(\boldsymbol{\delta}), \mathbb{Z}_{o}^{n-1}\right)$.

Now we will state two sufficient conditions for a merging operation to satisfy the holonomy constraints: one for simplicial points and one for non-simplicial points.

Lemma 2.33 (Non-simplicial merging). Let $n \geq 4$. Let $\boldsymbol{\delta} \in \diamond^{n}$ be non-simplicial and suppose that $\delta_{i}^{\pi}$ is an integer. Let $M$ be a merging operation of type $M_{(i+j)}, M_{(i-j)}, M_{(j-i)}$ with $j \neq i$. Then the point $M(\boldsymbol{\delta})$ lies is $\operatorname{int}\left(\mathcal{H}^{n-1}\right)$.

Proof. Let $\boldsymbol{c}$ be the center of $\diamond^{n}$. We will prove that the image of the segment $\left[\boldsymbol{\delta}^{\boldsymbol{\pi}}, \boldsymbol{c}\right]$ under the $\operatorname{map} M: \mathbb{R}^{n} \rightarrow \mathbb{R}^{n-1}$ lies in $\diamond^{n-1}$ and at worst one of its points, namely $M(\boldsymbol{c})$, sits at distance 1 from $\mathbb{Z}_{o}^{n-1}$.

Note first that $M\left(\left[\boldsymbol{\delta}^{\boldsymbol{\pi}}, \boldsymbol{c}\right]\right)$ belongs entirely to some unit integer cube $\square^{n-1}$. Indeed, $c_{i}+c_{j}$ and $c_{i}-c_{j}-2$ are integers, but $\left|\left(\delta_{i}^{\pi}+\delta_{j}^{\pi}\right)-\left(c_{i}+c_{j}\right)\right| \leq 1$ and $\left|\left(\delta_{i}^{\pi}-\delta_{j}^{\pi}-2\right)-\left(c_{i}-c_{j}-2\right)\right| \leq 1$. 
Moreover, both $M\left(\boldsymbol{\delta}^{\boldsymbol{\pi}}\right)$ and $M(\boldsymbol{c})$ satisfy holonomy constraints, i.e. they belong to some $\diamond^{n-1}$. Indeed, using the fact that $\delta_{i}^{\pi}$ is integer and using Lemma 2.31(a) and Lemma 2.32, we have

$$
d_{1}\left(M\left(\boldsymbol{\delta}^{\boldsymbol{\pi}}\right), \mathbb{Z}_{o}^{n-1}\right)=d_{1}\left(\boldsymbol{\delta}^{\boldsymbol{\pi}}, \mathbb{Z}_{o}^{n}\right)>1
$$

and so $M\left(\boldsymbol{\delta}^{\boldsymbol{\pi}}\right)$ does not belong to a simplicial face of $\diamond^{n-1}$. At the same time, $d_{1}\left(M(\boldsymbol{c}), \mathbb{Z}_{o}^{n-1}\right)=$ $\frac{n-2}{2} \geq 1$, since each coordinate of $\boldsymbol{c}$ is half-integral.

Since $\diamond^{n-1}$ is convex, and both ends of $M\left(\left[\boldsymbol{\delta}^{\boldsymbol{\pi}}, \boldsymbol{c}\right]\right)$ belong to the same $\diamond^{n-1}$, the whole segment belongs to it as well. On the other hand, $M\left(\boldsymbol{\delta}^{\boldsymbol{\pi}}\right)$ does not belong to a simplicial face of $\diamond^{n-1}$ and so at worst one point of the segment $M\left(\left[\boldsymbol{\delta}^{\boldsymbol{\pi}}, \boldsymbol{c}\right]\right)$ belongs to a simplicial face, namely $M(\boldsymbol{c})$. Clearly, this happens only if $n=4$.

Lemma 2.34 (Simplicial merging). Let $n \geq 5$. Let $\boldsymbol{\delta} \in \diamond^{n}$ be simplicial and let $\boldsymbol{m}$ be a corresponding odd vertex. Consider a merging operation $M$ of positive type $M_{(i+j)}$ or of negative type $M_{(i-j)}$ and suppose that $d_{1}(M(\boldsymbol{m}), M(\boldsymbol{\delta}))>1$. Then $M(\boldsymbol{\delta})$ belongs to int $\left(\mathcal{H}^{n-1}\right)$.

Proof. As in the proof of Lemma 2.33. note that the segment $M\left(\left[\boldsymbol{\delta}^{\boldsymbol{\pi}}, \boldsymbol{c}\right]\right)$ belongs to some unit integer cube $\square^{n-1}$. Let $\diamond^{n-1}$ be the truncated cube associated to such a $\square^{n-1}$.

Since $n \geq 5$, the point $M(\boldsymbol{c})$ does not belong to any simplicial face of $\diamond^{n-1}$, because $d_{1}\left(M\left(\boldsymbol{m}^{\prime}\right), M(\boldsymbol{c})\right)=$ $\frac{n-2}{2}>1$ for every vertex $\boldsymbol{m}^{\prime}$ of $\square^{n-1}$. From $d_{1}\left(M(\boldsymbol{m}), M\left(\boldsymbol{\delta}^{\boldsymbol{\pi}}\right)\right) \leq d_{1}\left(\boldsymbol{m}, \boldsymbol{\delta}^{\boldsymbol{\pi}}\right)=1$ we deduce that the segment $M\left(\left[\boldsymbol{\delta}^{\boldsymbol{\pi}}, \boldsymbol{c}\right]\right)$ intersects the simplicial face of $\diamond^{n-1}$ corresponding to the odd vertex $M(\boldsymbol{m})$. Let us denote such a point of intersection by $\boldsymbol{z}$ and observe that the point $M(\boldsymbol{c})$ is the center of a non-simplicial face of $\diamond^{n-1}$. Thus, the segment $[M(\boldsymbol{c}), \boldsymbol{z}]$ lies inside $\diamond^{n-1}$ and in fact it is not contained in any simplicial face of $\diamond^{n-1}$. As $M(\boldsymbol{\delta})$ lies in the interior of the segment $[M(\boldsymbol{c}), \boldsymbol{z}]$, the conclusion follows.

The following example shows why the restriction $n>4$ is important.

Example 2.35 (Case $n=4)$. Let $a \in\left(0, \frac{1}{2}\right)$ and let $\boldsymbol{\delta}=(a,-a,-1+a,-1+a) \in \mathbb{R}^{4}$. Observe that $\boldsymbol{\delta}$ is a defect vector that satisfies positivity and holonomy constraints strictly; nevertheless, only the mergings $M_{(1+2)}, M_{(1+3)}$ and $M_{(1+4)}$ preserve the positivity constraints. At the same time neither of these three positive mergings strictly preserves the holonomy constraints.

\subsubsection{Case (a): $\delta_{1} \leq 0$}

The following observation is elementary and so we omit the proof.

Lemma 2.36. The domain in $\mathbb{R}^{n}$ obtained by intersecting $\operatorname{int}\left(\mathcal{A}^{n}\right)$ with the cube $(-1,0]^{n}$ is described by the following system of $2 n+1$ inequalities:

$$
\begin{cases}\delta_{i} \leq 0 & \text { for all } i=1, \ldots, n \\ \sum_{j=1}^{n} \delta_{j}<2 \delta_{i} & \text { for all } i=1, \ldots, n \\ \sum_{j=1}^{n} \delta_{j}>-2 & \end{cases}
$$

In order to simplify the notation, up to rearranging the indices we will assume now on that $\delta_{1} \geq \delta_{2} \geq \cdots \geq \delta_{n}$.

Proposition 2.37. Assume $n \geq 5$. Suppose that $\boldsymbol{\delta} \in \operatorname{int}\left(\mathcal{A}^{n}\right) \cap(-1,0)^{n}$. Then $M_{(1+2)}(\boldsymbol{\delta}) \in$ $\operatorname{int}\left(\mathcal{A}^{n-1}\right)$.

Proof. Since $\sum_{j} \delta_{j}>-2$ and $n \geq 4$, we have $\delta_{1}+\delta_{2}>-1$. Hence $M_{(1+2)}(\boldsymbol{\delta})$ satisfies the positivity constraints.

Suppose now that $\boldsymbol{\delta}$ is a simplicial point. It is easy to see that the point $-\boldsymbol{e}_{\boldsymbol{n}}$ is an odd-integer point closest to $\boldsymbol{\delta}$. We have $d_{1}\left(M_{(1+2)}(\boldsymbol{\delta}), M_{(1+2)}\left(-\boldsymbol{e}_{\boldsymbol{n}}\right)\right)=d_{1}\left(\boldsymbol{\delta}, \boldsymbol{e}_{\boldsymbol{n}}\right)>1$. So by Lemma 2.34, the vector $M_{(1+2)}(\boldsymbol{\delta})$ strictly satisfies the holonomy constraints.

Suppose now that $\boldsymbol{\delta}$ is not a simplicial point, and so $\boldsymbol{\delta}^{\boldsymbol{\pi}}$ strictly satisfies the holonomy constraints and there exists an index $i$ such that $\delta_{i}^{\pi}$ is an integer. As $-\boldsymbol{e}_{\boldsymbol{i}}$ is an odd-integer vector, we have $1+2 \delta_{i}^{\pi}-\sum_{j} \delta_{j}^{\pi}=d_{1}\left(-\boldsymbol{e}_{\boldsymbol{i}}, \boldsymbol{\delta}^{\boldsymbol{\pi}}\right)>1$. On the other hand, $\sum_{j} \delta_{j}^{\pi}>-2$, because $\sum_{j} \delta_{j}>-2$ and 
$n \geq 4$. As a consequence, $2 \delta_{i}^{\pi}>\sum_{j} \delta_{j}^{\pi}>-2$ and so $\delta_{i}^{\pi}=0$. The assumption $\delta_{1} \geq \delta_{i}$ necessarily implies $\delta_{1}^{\pi} \geq \delta_{i}^{\pi}=0$ and so $\delta_{1}^{\pi}=0$, because $\delta^{\pi} \in[-1,0]^{n}$. By Lemma 2.33 we then conclude that $M_{(1+2)}(\boldsymbol{\delta})$ satisfies the holonomy constraints strictly.

\subsubsection{Case (b): $\delta_{1}>0$ and $\delta_{2}+\delta_{3}>-1$}

Up to rearranging the indices, assume that $\delta_{1} \geq \delta_{2} \geq \cdots \geq \delta_{n}$.

Proposition 2.38. Suppose that $n \geq 5$ and $\boldsymbol{\delta} \in \operatorname{int}\left(\mathcal{A}^{n}\right)$. Suppose moreover that $\delta_{1}>0$ and $\delta_{2}+\delta_{3}>-1$. Then there exist indices $i, j$ such that $M_{(i+j)}(\delta) \in \operatorname{int}\left(\mathcal{A}^{n-1}\right)$.

Proof. Note that a positive merging $M_{(i+j)}$ preserves the sum of the entries of the defect vector. Thus, in order to prove that $M_{(i+j)}(\boldsymbol{\delta})$ satisfies the positivity constraints, we only need to check that $\delta_{i}+\delta_{j}>-1$.

Suppose first that $\boldsymbol{\delta}$ is non-simplicial, and let $i$ be an index such that $\delta_{i}^{\pi}$ is integer. If $i \neq 1$, consider the positive merging $M_{(1+i)}$. According to Lemma 2.33 the vector $M_{(1+i)}(\boldsymbol{\delta})$ satisfies the holonomy constraints strictly. At the same time $\delta_{1}+\delta_{i}>-1$, because $\delta_{1}>0$. Similarly, if $i=1$, we can consider the merging $M_{(1+2)}$.

Suppose now that $\boldsymbol{\delta}$ is simplicial and let $\boldsymbol{m}$ be a closest odd-integer point. Chose $i$ and $j$ distinct elements of $\{1,2,3\}$ so that $m_{i}-\delta_{i}$ and $m_{j}-\delta_{j}$ are of the same sign. Then $d_{1}\left(M_{(i+j)}(\boldsymbol{m}), M_{(i+j)}(\boldsymbol{\delta})\right)=$ $d_{1}(\boldsymbol{m}, \boldsymbol{\delta})>1$ and so, according to Lemma 2.34, the vector $M_{(i+j)}(\boldsymbol{\delta})$ strictly satisfies the holonomy constraints. At the same time $\delta_{i}+\delta_{j}>-1$ by hypothesis and so positivity constraints are also satisfied.

\subsubsection{Case (c): $\delta_{1}>0$ and $\delta_{2}+\delta_{3} \leq-1$}

Notice in particular that, in such a case, $\delta_{j}<0$ for all $j>1$.

The following technical definition is useful to clarify when to apply a positive merging.

Definition 2.39. Let $n \geq 5$. A vector $\boldsymbol{\delta} \in \operatorname{int}\left(\mathcal{A}^{n}\right)$ with $\delta_{1} \geq \cdots \geq \delta_{n}$ is positively mergeable if the following three conditions are not simultaneously satisfied:

(a) the vector $\boldsymbol{\delta}$ is simplicial;

(b) there exists an integer $l \geq 1$ such that

(b1) $\boldsymbol{m}=(l,-1,-1, \ldots,-1)$ is a vector in $\mathbb{Z}_{o}^{n}$ closest to $\boldsymbol{\delta}$;

(b2) $l>\delta_{1}$;

(b3) $d_{1}\left(M_{(1+n)}(\boldsymbol{\delta}), M_{(1+n)}(\boldsymbol{m})\right) \leq 1$.

Proposition 2.40 (Positive merging). Let $n \geq 5$ and let $\boldsymbol{\delta} \in \operatorname{int}\left(\mathcal{A}^{n}\right)$ be a positively mergeable defect vector. Then there exists indices $i, j$ such that $M_{(i+j)}(\boldsymbol{\delta}) \in \operatorname{int}\left(\mathcal{A}^{n-1}\right)$.

Proof. Suppose first that $\delta$ is the center of a unit cube. Then all its entries are half-integers. Since $n \geq 5$, the vector $M_{(i+j)}(\boldsymbol{\delta})$ sits at distance $\geq \frac{3}{2}$ from $\mathbb{Z}_{o}^{n-1}$ for any distinct $i$ and $j$, and so $M_{(i+j)}(\boldsymbol{\delta}) \in \operatorname{int}\left(\mathcal{A}^{n-1}\right)$.

Thus, now on we can assume that $\delta$ is not the center of a unit cube. Thanks to Propositions 2.37 and 2.38 it is enough to treat the case $\delta_{1} \geq 0$ and $\delta_{2}+\delta_{3} \leq-1$.

Case (a) violated.

The vector $\boldsymbol{\delta}$ is non-simplicial. Consider the positive merging $M_{(1+j)}$, where either $\delta_{1}^{\pi}$ or $\delta_{j}^{\pi}$ is integer. Since $\delta_{1} \geq 0$, the vector $M_{(1+j)}(\boldsymbol{\delta})$ satisfies the positivity constraints; moreover, by Lemma 2.33 it also satisfies holonomy constraints strictly.

Assume now on that (a) is satisfied.

The vector $\boldsymbol{\delta}$ is simplicial: let $\boldsymbol{m}$ be a point in $\mathbb{Z}_{o}^{n}$ closest to $\boldsymbol{\delta}$, which is necessarily of the following type $\boldsymbol{m}=l \boldsymbol{e}_{\mathbf{1}}-\sum_{j \in J} \boldsymbol{e}_{j}$, for some integer $l \geq 0$ and some $J \subset\{2,3, \ldots, n\}$. 
Suppose $3 \notin J$, and so $2 \notin J$ either. Then $d_{1}(\boldsymbol{m}, \boldsymbol{\delta}) \geq d_{1}\left(\boldsymbol{m}-\boldsymbol{e}_{\mathbf{2}}-\boldsymbol{e}_{\mathbf{3}}, \boldsymbol{\delta}\right)$. Replacing $\boldsymbol{m}$ by $\boldsymbol{m}-\boldsymbol{e}_{\mathbf{2}}-\boldsymbol{e}_{\mathbf{3}}$, we can thus assume $3 \in J$ and then $\{3,4, \ldots, n\} \subset J$.

Assume now on that either $\boldsymbol{m}=l \boldsymbol{e}_{\mathbf{1}}-\left(\boldsymbol{e}_{\mathbf{2}}+\cdots+\boldsymbol{e}_{\boldsymbol{n}}\right)$ or $\boldsymbol{m}=l \boldsymbol{e}_{\mathbf{1}}-\left(\boldsymbol{e}_{\mathbf{3}}+\cdots+\boldsymbol{e}_{\boldsymbol{n}}\right)$.

If $\delta_{1}-m_{1}=\delta_{1}-l \geq 0$, then $d_{1}\left(M_{1+3}(\boldsymbol{\delta}), M_{1+3}(\boldsymbol{m})\right)=d_{1}(\boldsymbol{\delta}, \boldsymbol{m})>1$. Thus, $M_{(1+3)}(\boldsymbol{\delta})$ strictly satisfies the holonomy constraints by Lemma 2.34.

If $\delta_{1}-l<0$ and $m_{2}=0$, then $d_{1}\left(M_{1+2}(\boldsymbol{\delta}), M_{1+2}(\boldsymbol{m})\right)=d_{1}(\boldsymbol{\delta}, \boldsymbol{m})>1$. As above, $M_{(1+2)}(\boldsymbol{\delta})$ strictly satisfied the holonomy constraints by Lemma 2.34

Thus, we are left to deal with the case $\delta_{1}<l$ and $m_{2}=-1$.

Assume now on that (b1) and (b2) are satisfied.

Since $d_{1}\left(M_{(1+i)}(\boldsymbol{m}), M_{(1+i)}(\boldsymbol{\delta})\right) \leq d_{1}\left(M_{(1+j)}(\boldsymbol{m}), M_{(1+j)}(\boldsymbol{\delta})\right)$ for all $2 \leq i<j \leq n$, we can again conclude by applying Lemma 2.34 to the operation $M=M_{(1+n)}$, unless $d_{1}\left(M_{(1+n)}(\boldsymbol{m}), M_{(1+n)}(\boldsymbol{\delta})\right) \leq$ 1 , that is unless $\boldsymbol{\delta}$ is not positively mergeable.

The remaining cases can be taken care of by negative merging.

Proposition 2.41 (Negative merging). Suppose that $n \geq 5$ and $\boldsymbol{\delta}$ is not positively mergeable. Then $M_{(1-n)}(\boldsymbol{\delta})$ satisfies positivity and strict holonomy constraints. Moreover, $\delta_{1}, \delta_{n}$ and $\delta_{1}-\delta_{n}$ are not integers.

Proof. By our assumptions,

$$
\begin{aligned}
1 \geq d_{1}\left(M_{(1+n)}(\boldsymbol{\delta}), M_{(1+n)}(\boldsymbol{m})\right) & =\left|1-l+\delta_{1}+\delta_{n}\right|+(n-2)+\sum_{1<j<n} \delta_{j} \geq \\
& \geq n-l-1+\sum_{j} \delta_{j}
\end{aligned}
$$

that is, $\sum_{j} \delta_{j} \leq 2+l-n$. Since $\sum \delta_{j}>-2$, we conclude that $n-l<4$. Since $\boldsymbol{m}$ is odd, the integer $n-l$ is even and so $l \geq n-2$. Moreover $\delta_{1} \geq l-1$, because $\boldsymbol{m}$ is a vector in $\mathbb{Z}_{o}^{n}$ closest to $\delta$.

Recall that $M_{(1-n)}(\boldsymbol{\delta})=\left(\delta_{2}, \ldots, \delta_{n-1}, \delta_{1}-\delta_{n}-2\right)$. By the above computations,

$$
\delta_{1}-\delta_{n}-2 \geq(l-1)-\delta_{n}-2 \geq n-5-\delta_{n}>-1
$$

and

$$
\begin{aligned}
\delta_{2}+\cdots+\delta_{n-1}+\left(\delta_{1}-\delta_{n}-2\right) & \geq \delta_{1}+\cdots+\delta_{n-2}-2 \geq \delta_{1}+(n-3) \delta_{n-2}-2> \\
& >(l-1)-(n-3)-2 \geq-2
\end{aligned}
$$

which shows that $M_{(1-n)}(\boldsymbol{\delta})$ satisfies the positivity constraints.

On the other hand,

$$
\begin{aligned}
d_{1}\left(M_{(1-n)}(\boldsymbol{\delta}), M_{(1-n)}(\boldsymbol{m})\right) & =\left|(l+1)-\left(\delta_{1}-\delta_{n}\right)\right|+\sum_{1<j<n}\left(1+\delta_{j}\right)= \\
& =\left(l-\delta_{1}\right)+\sum_{j>1}\left(1+\delta_{j}\right)=d_{1}(\boldsymbol{\delta}, \boldsymbol{m})>1
\end{aligned}
$$

and so $M_{(1-n)}(\boldsymbol{\delta})$ strictly satisfies the holonomy constraints by Lemma 2.34.

Since $\boldsymbol{\delta}$ is not positively mergeable, it is easy to see that $\delta_{1}$ and $\delta_{n}$ cannot be integers. In order to show that $\delta_{1}-\delta_{n}$ is not an integer either, it is enough to prove that $\delta_{1}-\delta_{n}>l$, because $\delta_{1}<l$ and $\delta_{n} \in(-1,0)$. This can be easily verified, since

$$
\begin{aligned}
& 1 \geq\left|1-l+\delta_{1}+\delta_{n}\right|+(n-2)+\sum_{1<j<n} \delta_{j} \geq \\
& \quad \geq l-1-\delta_{1}-\delta_{n}+(n-2)\left(1+\delta_{n}\right)>\left(l-\delta_{1}+\delta_{n}\right)+1
\end{aligned}
$$

and so $\delta_{1}-\delta_{n}>l$.

So finally we have achieved our task. 
Proof of Theorem 2.27. If $\boldsymbol{\delta}$ is positively mergeable or the center of a unit cube, then a positive merging operation will work by Proposition 2.40 and so (a) holds. On the other hand, if $\boldsymbol{\delta}$ is not positively mergeable, then Proposition 2.41 ensures that a negative operation of type $M_{(i-j)}$ works and that, in this case, the involved defects $\delta_{i}, \delta_{j}$ and $\delta_{i}-\delta_{j}$ are not integers. Thus, (b) holds.

\section{Geometric constructions}

\subsection{Spherical bigons and triangles}

Definition 3.1. Let $n \geq 2$. A spherical $n$-gon is a bordered surface homeomorphic to the closed unit disc, endowed with a Riemannian metric of constant curvature 1, whose boundary consists of $n$ geodesic arcs (called edges) that form inner angles $\pi \vartheta_{1}, \ldots, \pi \vartheta_{n}$. We will say that such an $n$-gon is convex if all $\vartheta_{i} \leq 1$.

Mimicking Definition 2.8, we can consider angle-deformability of spherical $n$-gons.

Definition 3.2. A metric $g$ on a spherical $n$-gon with inner angles $\pi \cdot \boldsymbol{\vartheta}$ is angle-deformable if there exists a neighbourhood $\mathcal{N}$ of $\boldsymbol{\vartheta} \in \mathbb{R}^{n}$ and a continuous family $\mathcal{N} \ni \boldsymbol{\nu} \mapsto g_{\boldsymbol{\nu}}$ of spherical metrics on the $n$-gon such that $g_{\boldsymbol{\nu}}$ has conical singularities of angles $\pi \cdot \boldsymbol{\nu}$ for all $\boldsymbol{\nu} \in \mathcal{N}$ and $g_{\vartheta}=g$.

We will refer to a 2-gon, 3-gon and 4-gon respectively as a "bigon", "triangle" and "quadrilateral". If $x_{i}, x_{i+1}$ are consecutive vertices of an $n$-gon, then we will denote by $\left|x_{i} x_{i+1}\right|$ the length of the edge joining them.

Notation. Let $S$ be a compact spherical surface possibly with boundary and let $\gamma$ be a curve inside $S$. We say that $S^{\prime}$ is obtained from $S$ by cutting along $\gamma$ if $S^{\prime}$ is the compact spherical surface (possibly with boundary) obtained as a metric completion of $S \backslash \gamma$.

Notation. Let $S$ be a compact spherical surface with boundary. The double $D S$ of $S$ is the spherical surface obtained by gluing $S$ with $\bar{S}$ (another copy of $S$, with the opposite orientation) isometrically along their boundary. We will say that $S$ is angle-deformable (resp. non-coaxial) if $D S$ is (resp. if $D S$ has non-coaxial holonomy).

\subsubsection{Bigons}

Pick spherical coordinates $\psi \in[0,2 \pi)$ and $\phi \in[0, \pi]$ on $\mathbb{S}^{2}$. Given $0<\alpha<1$ and $0<r \leq \pi$, we denote by $B_{\alpha}(r)=\{(\psi, \phi) \mid \psi \in[0, \pi \alpha]$ and $\phi \in[0, r)\}$ and by $\bar{B}_{\alpha}(r)$ its closure. For $\alpha \geq 1$, we let $B_{\alpha}(r)$ be obtained from $k$ copies $B_{1}, \ldots, B_{k}$ of $B_{\alpha / k}(r)$ by gluing one geodesic side of $B_{i}$ to one geodesic side of $B_{i+1}$ for $i=1, \ldots, k-1$. Analogously for $\bar{B}_{\alpha}(r)$.

Definition 3.3. Let $\alpha>0$ and $r \in(0, \pi)$. The standard (open) $r$-neighbourhood of a vertex of a spherical polygon of angle $\pi \alpha$ is the surface with boundary $B_{\alpha}(r)$. The standard (open) $r$ neighbourhood of a cone point of angle $2 \pi \alpha$ is the spherical surface $S_{\alpha}(r)$ obtained by doubling $B_{\alpha}(r)$. In an analogous way we define the standard closed $r$-neighbourhoods.

Lemma 3.4 (Existence of bigons). (a) For every $\alpha>0$, there exists a bigon $B_{\alpha}$ with both angles $\pi \alpha$ and with cone points at distance $\pi$. Such $B_{\alpha}$ is angle-deformable.

(b) Let $d>0$ be an integer. There exists a continuous family of bigons $(0,2 \pi) \ni \ell \mapsto B(d, \ell)$ with both angles $d \pi$ and the two sides of lengths $(\ell, 2 \pi-\ell)$ for $d$ is odd, or $(\ell, \ell)$ for $d$ even.

Notation. In what follows, we will refer to a bigon to type (a) above as an (ordinary) bigon and to a bigon of type (b) as an exceptional bigon.

Proof of Lemma 3.4. The bigon in (a) is clearly $B_{\alpha}=\bar{B}_{\alpha}(\pi)$ and deformability is obvious.

About claim (b), let $D \subset \mathbb{S}^{2}$ be a closed unit hemisphere. The bigon $B(1, \ell)$ is easily obtained from $D$ by marking two points $x_{1}, x_{2}$ on $\partial D$ that break $\partial D$ into two geodesic arcs of lengths $\ell$ and $2 \pi-\ell$. For $d>1$, consider cyclic cover $\tilde{S} \rightarrow \mathbb{S}^{2}$ of degree $d$ branched at $x_{1}, x_{2}$. If $B_{(1)}, \ldots, B_{(d)}$ are the lifts of $D$ and $B_{(1)}^{\prime}, \ldots, B_{(d)}^{\prime}$ are the lifts of the other hemisphere $\overline{\mathbb{S}^{2} \backslash D}$, then $B(d, \ell)$ is obtained as the union of all $B_{(2 i+1)}$ and $B_{(2 i)}^{\prime}$.

By doubling the bigons constructed above, we immediately have the following. 
Corollary 3.5 (Spheres with two conical points). (a) For every $\alpha>0$, there exists a spherical surface $S_{\alpha}:=D B_{\alpha}$ homeomorphic to a sphere with both angles $2 \pi \alpha$ and with cone points at distance $\pi$. Such $S_{\alpha}$ are angle-deformable.

(b) Let $d>0$ be an integer. There exists a continuous family $(0, \pi) \ni \ell \mapsto D B(d, \ell)$ of spherical surfaces with cone points at distance $\ell$ and both angles $2 \pi d$.
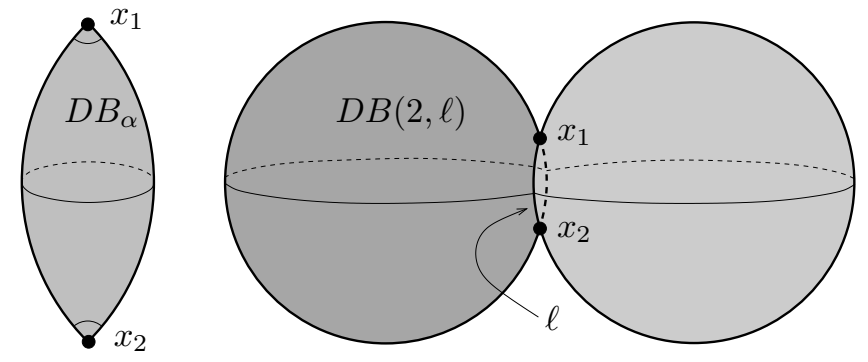

Figure 3: An ordinary and an exceptional sphere with two conical points.

Remark 3.6. It can be easily seen that all bigons are of types (a) and (b) described in Lemma 3.4. Analogously, surfaces of curvature 1 homeomorphic to a sphere with two conical points can be obtained by doubling such bigons as in Corollary 3.5 (see Troyanov [18).

As an application, here we characterize spherical surfaces with non-integral angles and reducible holonomy.

Lemma 3.7 (Metrics with reducible holonomy). Let $S$ be a spherical surface with conical singularities $x_{1}, \ldots, x_{n}$ of angles $2 \pi \vartheta_{1}, \ldots, 2 \pi \vartheta_{n}$. Suppose that the holonomy $\rho: \pi_{1}(\dot{S}) \rightarrow \operatorname{SO}(3, \mathbb{R})$ is reducible and that no $\vartheta_{i}$ is integral. Then there is a subset $J \subseteq\{1, \ldots, n\}$ and geodesic graph $G \in S$ such that

(a) $S \backslash G$ is the disjoint union of the disks $S_{\vartheta_{i}}(\pi / 2)$ for $i \in J^{c}$ and possibly of some hemispheres;

(b) for every $j \in J$ the conical point $x_{j}$ is contained in $G$ and has conical angle $2 \pi\left(k_{j}+\frac{1}{2}\right)$ for some $k_{j} \in \mathbb{Z}_{\geq 0}$.

Proof. Let $\widetilde{\dot{S}} \rightarrow \dot{S}$ be the universal cover. Consider the developing map dev $: \widetilde{\dot{S}} \rightarrow \mathbb{S}^{2}$ and the holonomy representation $\rho: \pi_{1}(\dot{S}) \rightarrow \mathrm{SO}(3, \mathbb{R})$ associated to the given spherical metric. Since $\rho$ is non-trivial and reducible, there is a plane $P \subset \mathbb{R}^{3}$ and an orthogonal line $L$ invariant under $\rho\left(\pi_{1}(\dot{S})\right)$. Clearly the map dev does not ramify over $\mathbb{S}^{2} \backslash(P \cup L)$.

Define $\widetilde{G}$ as the $\pi_{1}(\dot{S})$-invariant geodesic graph $\operatorname{dev}^{-1}\left(\mathbb{S}^{2} \cap P\right) \subset \widetilde{\dot{S}}$, which descends to a geodesic graph on $\dot{S}$. The closure $G$ of such graph passes through the conical points $\left\{x_{j} \mid j \in J\right\}$ for some $J \subseteq\{1, \ldots, n\}$.

Let $R_{j} \in \mathrm{SO}(3, \mathbb{R})$ be the holonomy along a loop that simply winds about $x_{j}$. Such an $R_{j}$ is not the identity and its axis lies in $P$; moreover, $R_{j}$ preserves $L$ : the only possibility is that $R_{j}$ is a rotation by an angle $\pi$ and so $\vartheta_{j}=k_{j}+\frac{1}{2}$ for some $k_{j} \in \mathbb{Z}_{\geq 0}$.

Consider now an $i \in J^{c}$. Let $D_{i}$ be the component of $S \backslash G$ that contains $x_{i}$ and let $\widetilde{\dot{D}}_{i} \rightarrow \dot{D}_{i}$ be the universal cover of $\dot{D}_{i}=D_{i} \backslash\left\{x_{i}\right\}$. The developing map restricts to a cover of $\dot{D}_{i}$ over a component of $\mathbb{S}^{2} \backslash(P \cup L)$ and so $D_{i}$ is isometric to $S_{\vartheta_{i}}(\pi / 2)$.

Let $D$ be a component of $S \backslash G$ that does not contain any $x_{i}$. Then dev induces an isomorphism between $D$ a component of $\mathbb{S}^{2} \backslash P$, and so $D$ is a hemisphere.

\subsubsection{Triangles}

The following theorem follows from [3, Theorem 3].

Theorem 3.8 (Existence of triangles). Let $\boldsymbol{\vartheta}=\left(\vartheta_{1}, \vartheta_{2}, \vartheta_{3}\right)$ be a triple of real numbers satisfying holonomy constraints (프) strictly and the positivity constraints (P). Then there exists an angledeformable non-coaxial spherical triangle with inner angles $\pi \cdot \boldsymbol{\vartheta}$. 
Since we will need spherical triangles later, here we give a short constructive proof of the above theorem. The wished triangle is assembled from pieces constructed in Lemma 3.4. Lemma 3.11 and Corollary 3.10 ,

Lemma 3.9 (Existence of convex triangles). Let $\boldsymbol{\vartheta}=\left(\vartheta_{1}, \vartheta_{2}, \vartheta_{3}\right) \in(0,1)^{3}$. A convex spherical triangle with angles $\pi \cdot \vartheta$ exists if and only if both conditions are satisfied:

(i) the numbers $\left(1-\vartheta_{1}, 1-\vartheta_{2}, 1-\vartheta_{3}\right)$ satisfy the triangular inequality;

(ii) the following inequality holds: $\vartheta_{1}+\vartheta_{2}+\vartheta_{3}-1>0$.

Moreover, such convex triangles are angle-deformable and non-coaxial.

Proof. Assume a convex spherical triangle with angles $\pi \cdot \boldsymbol{\vartheta}$ exists. Then condition (i) holds since its dual spherical triangle has edges of lengths $\pi\left(1-\vartheta_{i}\right)$. Moreover, (ii) holds too, since $\pi\left(\vartheta_{1}+\vartheta_{2}+\vartheta_{3}-1\right)$ is the area of the triangle. Vice versa, fix a hemisphere $D$ and a point $x_{2}$ on $\partial D$. It is easy to see that, for every triple $\left(\vartheta_{1}, \vartheta_{2}, \vartheta_{3}\right)$ satisfying (i) and (ii), one can realize a triangle with such angles as the convex hull in $D$ of $x_{1}, x_{2}, x_{3}$, for suitable $x_{3} \in \partial D$ and $x_{1} \in D$, see Figure 4 (a). Such triangles are angle-deformable by construction; moreover, since $\vartheta_{i} \in(0,1)$ and the triangle is inscribed in a hemisphere, it is immediate to see that it is non-coaxial.

This lemma settles Theorem 3.8 for triangles with $\vartheta_{i}<1$. Indeed, inside the unit cube $[0,1]^{3}$ Inequalities $(\underline{\mathrm{H}})$ and conditions (i) and (ii) describe the tetrahedron with vertices $(1,0,0),(0,1,0)$, $(0,0,1),(1,1,1)$.
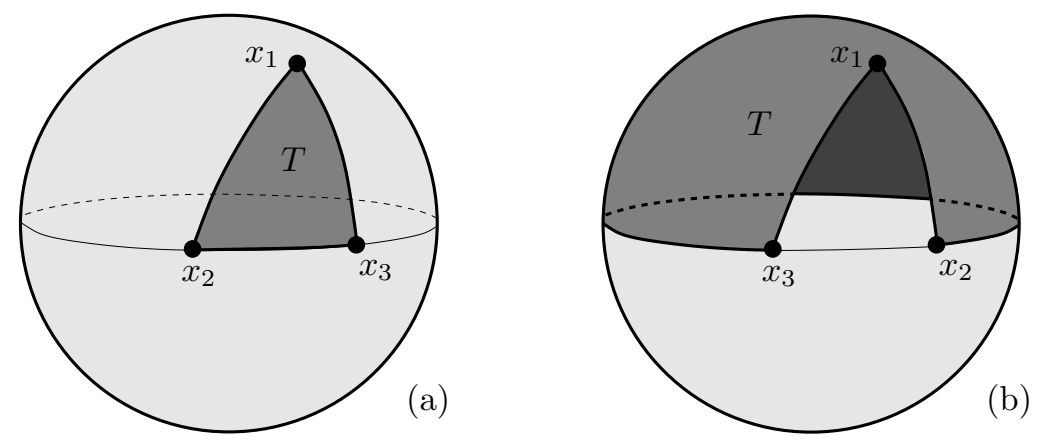

Figure 4: A convex triangle (a) and a complement (b) of a convex triangle in a hemisphere.

Corollary 3.10 (Triangles with small angles). Suppose $\boldsymbol{\vartheta}=\left(\vartheta_{1}, \vartheta_{2}, \vartheta_{3}\right)$ satisfy Inequalities (프) strictly and it belongs to the domain $\Pi^{3}:=[0,2] \times[0,1] \times[0,1] \subset \mathbb{R}^{3}$. Then there exists an angledeformable non-coaxial spherical triangle with angles $\pi \cdot \boldsymbol{\vartheta}$.

Proof. As we explained, the case when all $\vartheta_{i}$ are less than 1 follows from Lemma 3.9, Suppose that $1<\vartheta_{1}<2$. Then it is easy to check that the triple $\boldsymbol{\vartheta}^{\prime}=\left(2-\vartheta_{1}, 1-\vartheta_{3}, 1-\vartheta_{2}\right)$ satisfies Inequalities $(\underline{\mathrm{H}})$ as well. Consider a convex spherical triangle $T^{\prime} \subset \mathbb{S}^{2}$ with angles $\pi\left(\vartheta_{1}^{\prime}, \vartheta_{3}^{\prime}, \vartheta_{2}^{\prime}\right)$ at the vertices $\left(x_{1}, x_{3}, x_{2}\right)$ and let $E \subset \mathbb{S}^{2}$ be the maximal circle that contains the vertices $x_{2}$ and $x_{3}$. Cut $\mathbb{S}^{2}$ along $E$ and let $D$ be the component that contains $\operatorname{int}\left(T^{\prime}\right)$. Then the spherical triangle obtained from $D \backslash T^{\prime}$ by metric completion has angles $\pi \cdot \boldsymbol{\vartheta}$, see Figure 凹(b). Angle-deformability and non-coaxiality of $T^{\prime}$ implies that the constructed triangles is angle-deformable and non-coaxial too.
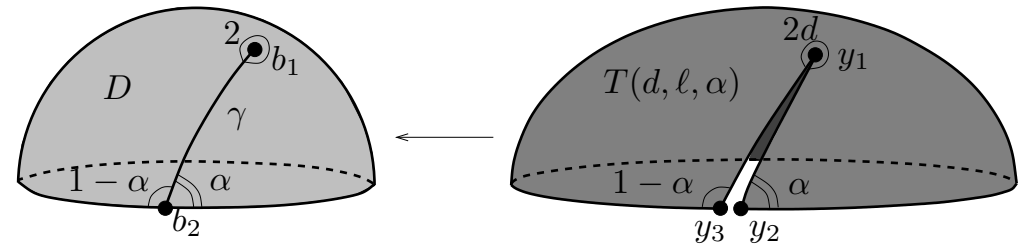

Figure 5: The triangle $T(d, \ell, \alpha)$. 
Lemma 3.11 (The triangles $T(d, \ell, \alpha)$ ). Let $d$ be a positive integer and let $0<\ell<\pi$ and $0<\alpha<1$. There exists a spherical triangle $T(d, \ell, \alpha)$ of vertices $y_{1}, y_{2}, y_{3}$ with edges of lengths $\left|y_{1} y_{2}\right|=\left|y_{1} y_{3}\right|=$ $\ell$ and $\left|y_{2} y_{3}\right|=2 \pi d$ and angles $\pi(2 d), \pi \alpha$, and $\pi(1-\alpha)$ at the vertices $y_{1}, y_{2}, y_{3}$ correspondingly.

Proof. Let $D \subset \mathbb{S}^{2}$ be a closed hemisphere. Choose a geodesic segment $\gamma$ on $D$ of length $\ell$, with one endpoint $b_{1}$ in the interior of $D$ and the other endpoint $b_{2}$ on the boundary of $D$ and forming angles $\pi \alpha$ and $\pi(1-\alpha)$ with $\partial D$. Consider now a ramified degree $d$ cover $\tilde{D} \rightarrow D$ that has an order $d$ branching at $b_{1}$. The wanted spherical triangle is obtained by cutting $\tilde{D}$ along $\tilde{\gamma}$, namely one of the $d$ geodesic preimages of $\gamma$, as illustrated in Figure 5

Denote by $\Gamma^{3} \subset \mathbb{Z}_{\geq 0}^{3}$ the additive semigroup consisting of elements $\boldsymbol{m}=\left(m_{1}, m_{2}, m_{3}\right)$ such that $m_{1} \geq m_{2} \geq m_{3}$ and $m_{1}+m_{2}+m_{3} \in 2 \mathbb{Z}$.

Lemma 3.12. The subset $\left\{\boldsymbol{\vartheta}=\left(\vartheta_{1}, \vartheta_{2}, \vartheta_{3}\right) \in \mathbb{R}^{3} \mid \vartheta_{1} \geq \vartheta_{2} \geq \vartheta_{3}\right\} \subset \mathbb{R}_{+}^{3}$ is contained in the union $\bigcup_{\boldsymbol{m} \in \Gamma^{3}}\left(\Pi^{3}+\boldsymbol{m}\right)$.

The previous lemma is completely elementary, and we omit the proof.

Proof of Theorem 3.8. Let $\boldsymbol{\vartheta}=\left(\vartheta_{1}, \vartheta_{2}, \vartheta_{3}\right) \in \mathbb{R}_{+}^{3}$ be a triple satisfying Inequalities (H) strictly. After reordering the coordinates, we can assume $\vartheta_{1} \geq \vartheta_{2} \geq \vartheta_{3}$. We will construct now a spherical triangle with angles $\pi \cdot \boldsymbol{\vartheta}$.

By Lemma 3.12, there exists $\boldsymbol{m} \in \Gamma^{3}$ and $\boldsymbol{\vartheta}^{\prime} \in \Pi^{3}$ such that $\boldsymbol{\vartheta}=\boldsymbol{\vartheta}^{\prime}+\boldsymbol{m}$. Since Inequality (H) is invariant with respect to translations by integer vectors $\boldsymbol{m}$ with $m_{1}+m_{2}+m_{3}$ even, by Corollary 3.10 there exists an angle-deformable non-coaxial spherical triangle $T^{\prime}$ with angles $\pi \vartheta_{1}^{\prime}, \pi \vartheta_{2}^{\prime}, \pi \vartheta_{3}^{\prime}$ at its vertices $x_{1}^{\prime}, x_{2}^{\prime}, x_{3}^{\prime}$. Since no $\vartheta_{i}^{\prime}$ is an integer, no edge of $T^{\prime}$ has length multiple of $\pi$.

Consider now separately two cases.

Case (a): $m_{1}>m_{2}+m_{3}$. The construction is illustrated in Figure 6

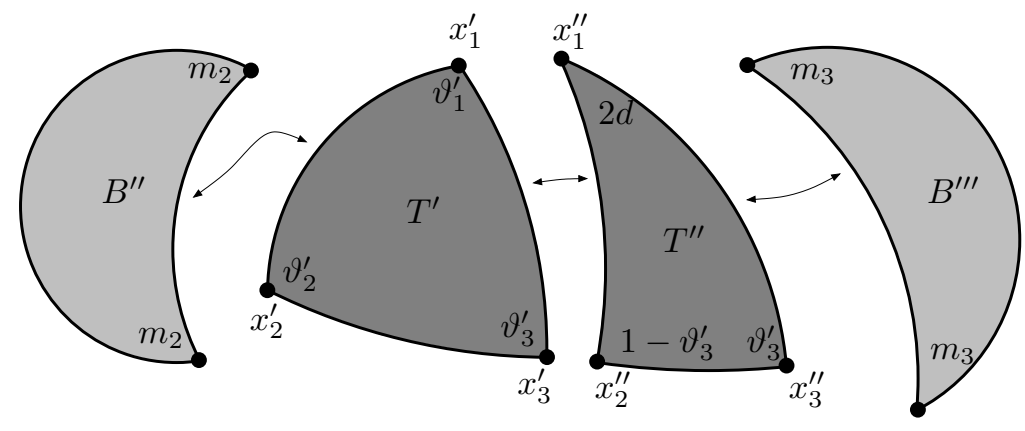

Figure 6: Building the triangle in case (a) of Theorem 3.8

Let $d=\frac{1}{2}\left(m_{1}-m_{2}-m_{3}\right)$. By Lemma 3.11 , there exists a spherical triangle $T^{\prime \prime}=T\left(d,\left|x_{1}^{\prime} x_{3}^{\prime}\right|, 1-\right.$ $\left.\vartheta_{3}^{\prime}\right)$ with vertices $x_{1}^{\prime \prime}, x_{2}^{\prime \prime}, x_{3}^{\prime \prime}$, angles $\pi\left(2 d, 1-\vartheta_{3}^{\prime}, \vartheta_{3}^{\prime}\right)$ and $\left|x_{1}^{\prime \prime} x_{2}^{\prime \prime}\right|=\left|x_{1}^{\prime \prime} x_{3}^{\prime \prime}\right|=\left|x_{1}^{\prime} x_{3}^{\prime}\right|$, Denote by $T$ the triangle with vertices $x_{1}, x_{2}, x_{3}$ obtained by identifying the side $x_{1}^{\prime \prime} x_{3}^{\prime \prime}$ of $T^{\prime \prime}$ with the side $x_{1}^{\prime} x_{3}^{\prime}$ of $T^{\prime}$. The angle at vertex $x_{1}$ of $T$ corresponding to $x_{1}^{\prime} \sim x_{1}^{\prime \prime}$ is $\pi\left(2 d+\vartheta_{1}^{\prime}\right)$, the angle at $x_{2}$ corresponding to $x_{2}^{\prime}$ is $\pi \vartheta_{2}^{\prime}$ and the angle at $x_{3}$ corresponding to $x_{3}^{\prime \prime}$ is $\pi \vartheta_{3}^{\prime}$. Finally, take two exceptional bigons $B^{\prime \prime}=B\left(m_{2},\left|x_{1} x_{2}\right|\right)$ and $B^{\prime \prime \prime}=B\left(m_{3},\left|x_{1} x_{3}\right|\right)$ and glue them with $T$ by isometrically identifying one side of $B^{\prime \prime}$ to $x_{1} x_{2}$ and one side of $B^{\prime \prime \prime}$ to $x_{1} x_{3}$. Angle-deformability of $T^{\prime}$ implies angledeformability of $T$ and so of the wished triangle. Since $\left|x_{1} x_{2}\right|=\left|x_{1}^{\prime} x_{2}^{\prime}\right|$ is not a multiple of $\pi$ and no $\vartheta_{i}$ is an integer, the triangle $T$ is non-coaxial and so is the constructed triangle.

Case (b): $m_{1} \leq m_{2}+m_{3}$. The construction is illustrated in Figure 7 


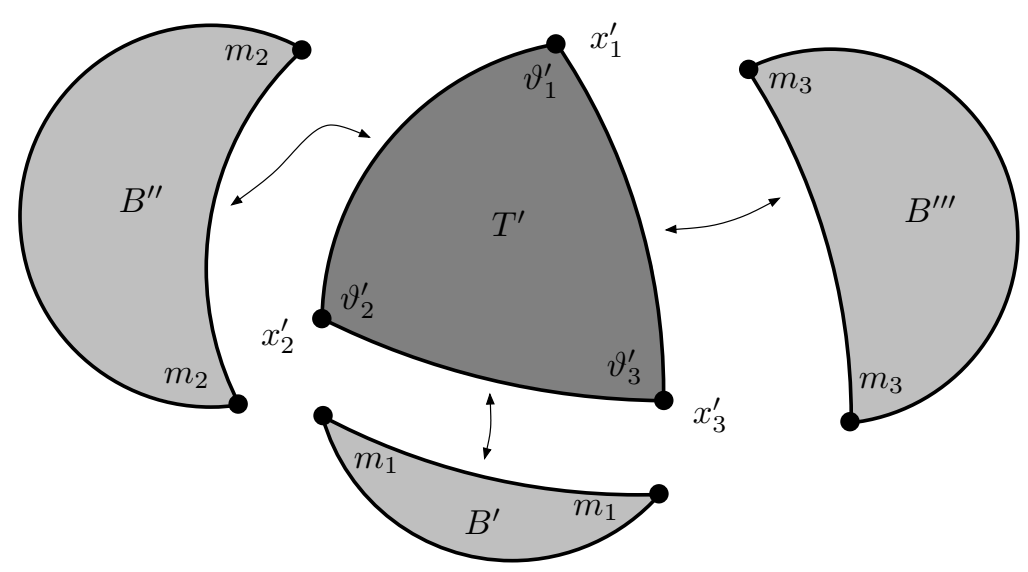

Figure 7: Building the triangle in case (b) of Theorem 3.8.

In this case, set $d_{1}=\frac{1}{2}\left(m_{2}+m_{3}-m_{1}\right), d_{2}=\frac{1}{2}\left(m_{3}+m_{1}-m_{2}\right), d_{3}=\frac{1}{2}\left(m_{1}+m_{2}-m_{3}\right)$. Take the three exceptional bigons $B^{\prime}=B\left(d_{1},\left|x_{2}^{\prime} x_{3}^{\prime}\right|\right), B^{\prime \prime}=B\left(d_{2},\left|x_{3}^{\prime} x_{1}^{\prime}\right|\right), B^{\prime \prime \prime}=B\left(d_{3},\left|x_{1}^{\prime} x_{2}^{\prime}\right|\right)$ and glue them with $T^{\prime}$ by isometrically identifying a side of $B^{\prime}$ with $x_{2}^{\prime} x_{3}^{\prime}$, a side of $B^{\prime \prime}$ with $x_{3}^{\prime} x_{1}^{\prime}$ and a side of $B^{\prime \prime \prime}$ with $x_{1}^{\prime} x_{2}^{\prime}$. As before, angle-deformability and non-coaxiality of $T^{\prime}$ implies angle-deformability and non-coaxiality of the constructed triangle.

The above existence theorem for triangle allows to draw the following conclusion about 3punctured spheres.

Corollary 3.13 (Existence of 3-punctured spherical metrics). Let $\boldsymbol{\vartheta}=\left(\vartheta_{1}, \vartheta_{2}, \vartheta_{3}\right)$ be a triple of real numbers satisfying holonomy (H) strictly and the positivity constraints (P). Then there exists an angle-deformable non-coaxial spherical surface $S$ of genus 0 with conical singularities of angle $2 \pi \cdot \vartheta$.

Proof. The wished spherical surface is obtained by doubling a spherical triangle with angles $\pi \cdot \boldsymbol{\vartheta}$, whose existence relies on Theorem 3.8 ,

Indeed, a little more is true.

Lemma 3.14 (Double of spherical triangles). Let $S$ be a sphere with distinct points $x_{1}, x_{2}, x_{3}$ endowed with a spherical metric $g$ with conical singularities of angle $2 \pi \vartheta_{i}$ at $x_{i}$ and non-coaxial holonomy. Then:

(a) $g$ is the unique spherical metric in its conformal class with such conical singularities;

(b) the spherical surface $(S, g)$ is obtained by doubling a spherical triangle.

Proof. The uniqueness of $g$ claimed in (a) was already noticed in [3. In fact, let $g^{\prime}$ be a spherical metric conformal to $g$ and denote by $J$ the underlying conformal structure. Both $g$ and $g^{\prime}$ induce $\mathbb{C P}^{1}$-structures $\Xi, \Xi^{\prime}$ on the Riemann surface $(\dot{S}, J)$ : their difference is thus encoded in a Schwarzian derivative $\sigma\left(\Xi, \Xi^{\prime}\right)$, which is a holomorphic quadratic differential on $\dot{S}$. A direct computation shows that $\sigma\left(\Xi, \Xi^{\prime}\right)$ has at most simple poles at $x_{1}, x_{2}, x_{3}$, because $g$ and $g^{\prime}$ have the same angles at the $x_{i}$, and so $\sigma\left(\Xi, \Xi^{\prime}\right) \equiv 0$. This implies that the two $\mathbb{C P}^{1}$-structures and so their holonomy representations agree. Moreover, the developing maps of $g$ and $g^{\prime}$ are conjugate through a Möbius transformation $\tau \in \operatorname{PSL}(2, \mathbb{C})$ that commutes with the holonomy subgroup of $\operatorname{SO}(3, \mathbb{R})$. Since we assumed the $\mathrm{SO}(3, \mathbb{R})$-holonomy to be non-coaxial, Lemma 2.17 (b) ensures that $\tau$ must lie in $\mathrm{SO}(3, \mathbb{R})$ and so $g=g^{\prime}$.

As for (b), we remark that $(S, J)$ is biholomorphic to $\mathbb{C P}^{1}$ through a map that takes $x_{1}, x_{2}, x_{3} \in S$ to $[1: 0],[1: 1],[0: 1] \in \mathbb{C P}^{1}$. The conjugation is an anti-holomorphic (and so conformal) transformation of $\mathbb{C P}^{1}$ that fixes $[1: 0],[1: 1],[0: 1]$ and so transports to a conformal involution $\iota$ of $S$ that fixes $x_{1}, x_{2}, x_{3}$. By (a), the metric $g$ must be fixed by $\iota$, which is thus an isometry of $(S, g)$. It is then immediate to check that $S$ is isometric to the double $D T$, where $T$ is the spherical triangle $S / \iota$. 


\subsubsection{Almost degenerate triangles}

Spherical triangles can degenerate in several ways. We are interested in describing two such degenerations: in the first case, the triangle degenerates to an ordinary bigon; in the second case, the triangle degenerates to a "double bigon", that is the union of two ordinary bigons sharing a common vertex.

Definition 3.15. A spherical polygon is $r$-wide at a vertex $x_{i}$ of angle $\pi \alpha$ if the closed ball centered at $x_{i}$ of radius $r$ is isometric to $\bar{B}_{\alpha}(r)$ and does not contain any marked point other than $x_{i}$. A spherical surface if $r$-wide at a cone point $x_{i}$ of angle $2 \pi \alpha$ if the closed ball centered at $x_{i}$ of radius $r$ is isometric to $\bar{S}_{\alpha}(r)$ and does not contain any marked point other than $x_{i}$.

Notation. If a spherical surface $S$ is $r$-wide at a conical point $y$ of angle $2 \pi \alpha$, then we denote by $U_{y}(r)$ the complement in $S$ of the open neighbourhood of $y$ isometric to $B_{\alpha}(r)$.

The triangles we are going to describe are needed in the surgery operations that will split a conical point into a pair of conical singularities. In order to prove the angle-deformability of the so-constructed spherical surface, we need the following properties from our triangles.

Definition 3.16. A spherical triangle $(T, g)$ with vertices $x_{1}, x_{2}, x_{3}$ and angles $\pi \cdot \boldsymbol{\vartheta}=\pi\left(\vartheta_{1}, \vartheta_{2}, \vartheta_{3}\right)$ is $\left(x_{1}, x_{2}\right)$-angle-deformable if there exists a neighbourhood $\mathcal{N}^{\prime} \subset \mathbb{R}^{2}$ of $\left(\vartheta_{1}, \vartheta_{2}\right)$, a continuous map $\theta_{3}: \mathcal{N}^{\prime} \rightarrow \mathbb{R}$ such that $\theta_{3}\left(\vartheta_{1}, \vartheta_{2}\right)=\vartheta_{3}$ and a continuous family of metrics $g_{\boldsymbol{\nu}}$ parametrized by $\boldsymbol{\nu} \in \mathcal{N}^{\prime}$ such that $g_{\left(\vartheta_{1}, \vartheta_{2}\right)}=g$ and $g_{\boldsymbol{\nu}}$ has angles $\pi\left(\nu_{1}, \nu_{2}, \theta_{3}\left(\nu_{1}, \nu_{2}\right)\right)$.

Notice that angle-deformability is clearly stronger than $\left(x_{1}, x_{2}\right)$-angle-deformability. On the other hand, the above definition is particularly meaningful for a $\vartheta$ that only weakly satisfies the holonomy constraints, in which case absolute angle-deformability cannot hold.

Also, we recall that a spherical surface of genus 0 with 3 conical points is obtained by doubling a spherical triangle. Thus, angle-deformability of the surface is equivalent to angle-deformability of the triangle.

Proposition 3.17 (Triangles close to an ordinary bigon). Let $\vartheta_{1}, \vartheta_{2}, \vartheta_{3}>0$ with $\vartheta_{3}=\vartheta_{1}+\vartheta_{2}-1$. For every $\varepsilon>0$ there exist $\eta \in(-\varepsilon, \varepsilon)$ and a spherical triangle $T$ with angles $\pi\left(\vartheta_{1}, \vartheta_{2}, \vartheta_{3}+\eta\right)$ and vertices $x_{1}, x_{2}, x_{3}$, which is $\pi(1-\varepsilon)$-wide at $x_{3}$ and $\left(x_{1}, x_{2}\right)$-angle-deformable.

Proof. We divide the proof into four cases, illustrated in Figure 8 .

(a)

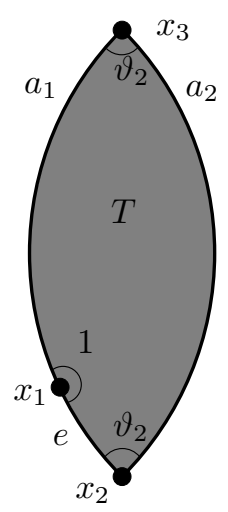

(b)

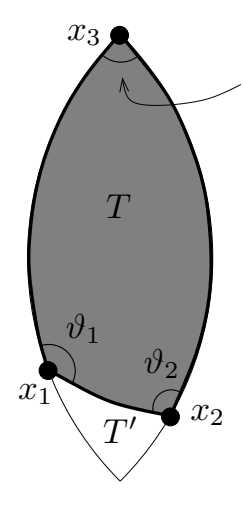

(c)

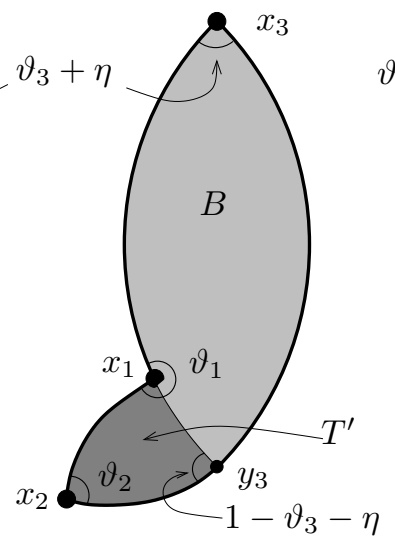

(d)

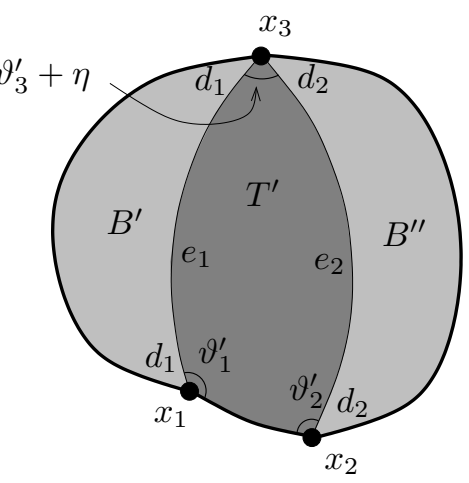

Figure 8: Triangles close to ordinary bigons.

Case (a): $\vartheta_{1}=1$.

Then we can take $T=B_{\vartheta_{2}}$, mark the two vertices of the ordinary bigon by $x_{2}, x_{3}$ and place $x_{1}$ on $\partial T$ at distance $\pi-\frac{\varepsilon}{2}$ from $x_{3}$. For such a $T$, we have $\eta=0$ and both edges $x_{1} x_{3}$ and $x_{2} x_{3}$ have length at most $\pi$.

Now, keep the edge $e$ from $x_{1}$ to $x_{2}$ fixed. For $i=1,2$ and for every $\nu_{i}$ close enough to $\vartheta_{i}$ shoot the geodesic arc $a_{i}$ starting from $x_{i}$ and that forms an angle $\pi \nu_{i}$ with $e$. Let $x_{3}$ be the first intersection 
of $a_{1}$ and $a_{2}$ and let $T_{\left(\nu_{1}, \nu_{2}\right)}$ the triangle bounded by $e, a_{1}, a_{2}$ and with internal angles $\pi\left(\nu_{1}, \nu_{2}, \theta_{3}\right)$, where $\theta_{3}$ is clearly a continuous function of $\left(\nu_{1}, \nu_{2}\right)$. It is easy to see that $\left(\nu_{1}, \nu_{2}\right) \mapsto T_{\left(\nu_{1}, \nu_{2}\right)}$ is a continuous family of triangles and that $T_{\left(\vartheta_{1}, \vartheta_{2}\right)}=T$. Hence, $T$ is $\left(x_{1}, x_{2}\right)$-deformable.

Case (b): $\vartheta_{1}, \vartheta_{2}<1$.

Let $\eta>0$ be smaller than $2\left(1-\vartheta_{1}\right), 2\left(1-\vartheta_{2}\right), 1-\vartheta_{3}$ so that the triple $\left(\vartheta_{1}, \vartheta_{2}, 1-\vartheta_{3}-\eta\right) \in(0,1)^{3}$ satisfies the triangular inequality. By Lemma 3.9, there exists a convex triangle $T^{\prime}$ with angles $\pi\left(1-\vartheta_{1}, 1-\vartheta_{2}, \vartheta_{3}+\eta\right)$ and vertices $\left(x_{1}, x_{2}, y_{3}\right)$. By construction, such a $T^{\prime}$ is embedded inside an ordinary bigon $B_{\vartheta_{3}+\eta}$ with vertices $y_{3}, x_{3}$. The closure $T$ of the complement of $T^{\prime}$ inside $B_{\vartheta_{3}+\eta}$ is a triangle vertices $x_{1}, x_{2}, x_{3}$, angles $\pi\left(\vartheta_{1}, \vartheta_{2}, \vartheta_{3}+\eta\right)$ and $\left|x_{1} x_{3}\right|,\left|x_{2} x_{3}\right|<\pi$. Notice that, as $\eta \rightarrow 0$, the area of $T^{\prime}$ (which depends on $\eta$ ) goes to zero and so its diameter goes to zero too (because $\vartheta_{1}, \vartheta_{2} \in(0,1)$ are fixed). Hence, for a sufficiently small $\eta$, the triangle $T$ is also $\pi(1-\varepsilon)$-wide at $x_{3}$. Since the holonomy of $D T$ is clearly non-coaxial, it is angle-deformable and so in particular $T$ is $\left(x_{1}, x_{2}\right)$-angle-deformable.

Case (c): $\vartheta_{1} \in(1,2), \vartheta_{2} \in(0,1)$ and $\vartheta_{3} \in(0,1]$.

Let $\eta<0$ so that $|\eta|$ is smaller than $\varepsilon, 2\left(\vartheta_{1}-1\right)$ and $2 \vartheta_{2}$. Thus, the triple $\left(2-\vartheta_{1}, 1-\vartheta_{2}, \vartheta_{3}+\eta\right) \in$ $(0,1)^{3}$ satisfies the triangular inequality and by Lemma 3.9 there exists a strictly convex triangle $T^{\prime}$ with vertices $x_{1}, x_{2}, y_{3}$ and angles $\pi\left(\vartheta_{1}-1, \vartheta_{2}, 1-\vartheta_{3}-\eta\right)$. By construction, $\left|x_{2} y_{3}\right|<\pi$. The triangle $T$ is the obtained by gluing $T^{\prime}$ with a standard bigon $B=B_{\vartheta_{3}+\eta}$ with vertices $x_{3}, y_{3}^{\prime}$ in such a way that $y_{3}^{\prime}$ is identified to $y_{3}$ and $e_{2}$ is glued to a portion of an edge of $B_{\vartheta_{3}+\eta}$. Thus, $\left|x_{1} x_{3}\right|<\pi$ and $\left|x_{2} x_{3}\right|<2 \pi$. As before, it is clear that the length of $x_{1} y_{3}$ goes to zero as $\eta \rightarrow 0$. Thus, $T$ is $\pi(1-\varepsilon)$-wide at $x_{3}$ for $|\eta|$ small enough. As above, $D T$ is non-coaxial and so angle-deformable, hence $T$ is $\left(x_{1}, x_{2}\right)$-angle-deformable.

Case (d): $\vartheta_{3}>1$.

Let $d_{1}, d_{2}$ be positive integers such that $\vartheta_{1}^{\prime}=\vartheta_{1}-d_{1} \in(0,2), \vartheta_{2}^{\prime}=\vartheta_{2}-d_{2} \in(0,1]$ and $\vartheta_{3}^{\prime}=$ $\vartheta_{3}-\left(d_{1}+d_{2}\right) \in(0,1]$. By cases (a) or (b), there exists an $\left(x_{1}, x_{2}\right)$-angle deformable triangle $T^{\prime}$ with angles $\pi\left(\vartheta_{1}^{\prime}, \vartheta_{2}^{\prime}, \vartheta_{3}^{\prime}+\eta\right)$ for some $|\eta|<\varepsilon$, which is $\pi(1-\varepsilon)$-wide at $x_{3}$. Call $e_{1}, e_{2}$ the edges $x_{1} x_{3}$ and $x_{2} x_{3}$ of $T^{\prime}$, of lengths $\ell_{1}, \ell_{2} \in \pi(1-\varepsilon, 2)$. The triangle $T$ is then obtained by gluing an edge of the exceptional bigon $B\left(d_{1}, \ell_{1}\right)$ with $e_{1}$ and an edge of $B\left(d_{2}, \ell_{2}\right)$ with $e_{2}$. Because $B\left(d_{1}, \ell_{1}\right)$ and $B\left(d_{2}, \ell_{2}\right)$ are $\pi(1-\varepsilon)$-wide at their vertices, such a $T$ is $\pi(1-\varepsilon)$-wide at $x_{3}$. Since this gluing procedure can be performed in families, the obtained triangle $T$ is $\left(x_{1}, x_{2}\right)$-angle-deformable.

Proposition 3.18 (Triangles close to a double bigon). Let $\vartheta_{1}, \vartheta_{2}>0$ with $\vartheta_{3}=\vartheta_{1}-\vartheta_{2}-1 \geq 0$ and assume that $\vartheta_{2}$ is not an integer. Then for every $\varepsilon>0$ there exist $\eta \in(-\varepsilon, \varepsilon)$ and a spherical triangle $T$ with angles $\pi\left(\vartheta_{1}, \vartheta_{2}, \vartheta_{3}+\eta\right)$ and vertices $x_{1}, x_{2}, x_{3}$, which is $\pi(1-\varepsilon)$-wide at $x_{3}$ and $\left(x_{1}, x_{2}\right)$-angle-deformable.

Proof. Again we divide the proof in four cases, illustrated in Figure 9 . 


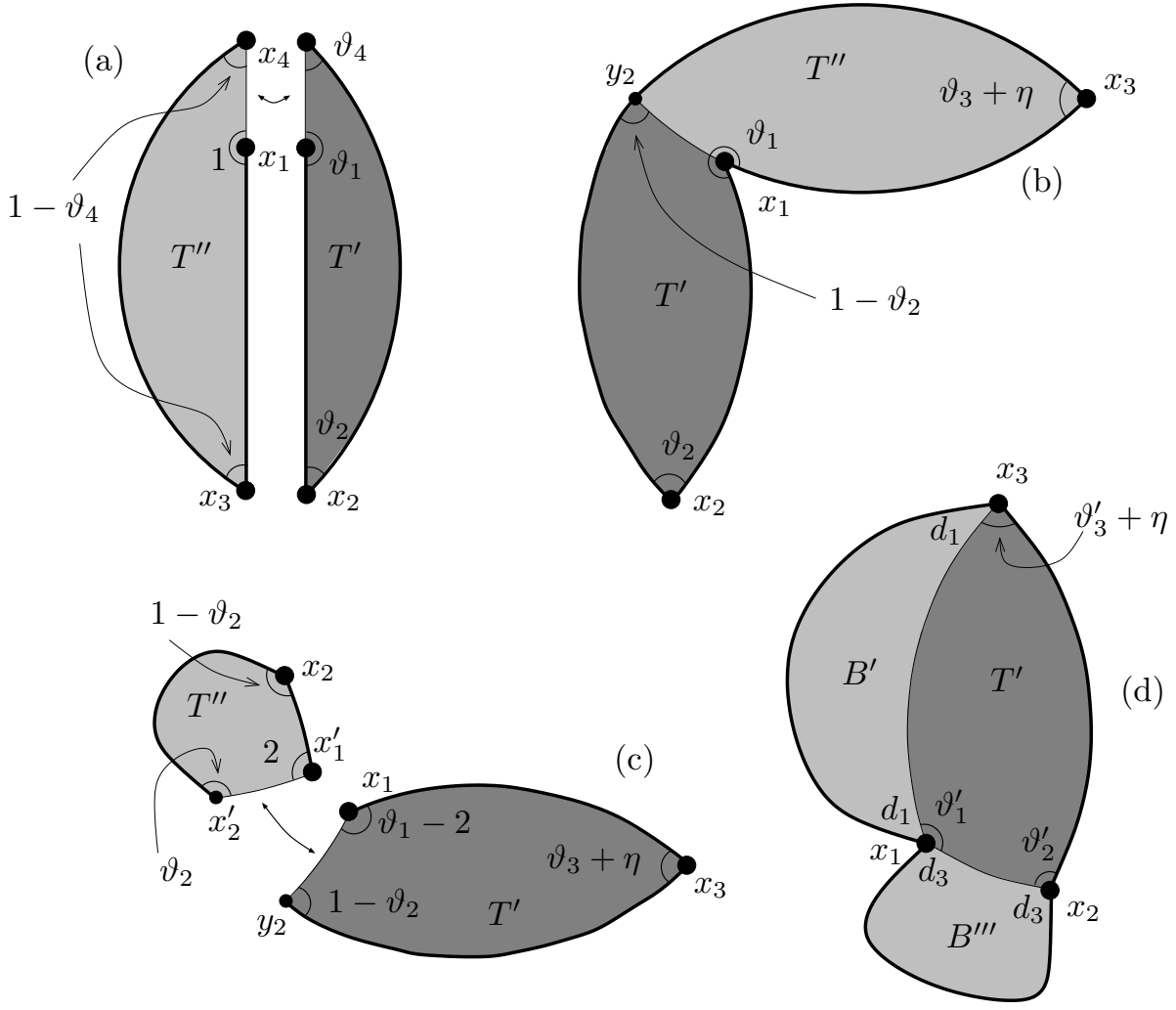

Figure 9: Triangles close to double bigons.

Case (a): $\vartheta_{2} \in(0,1), \vartheta_{1}=2$.

In this case $\vartheta_{3}=1-\vartheta_{2}>0$. Then $T$ can be chosen to be the triangle $T\left(1, \pi(1-\varepsilon / 2), \vartheta_{2}\right)$ constructed in Lemma 3.11. Notice that, in this case, $\eta=0$ and that both edges $x_{1} x_{2}$ and $x_{1} x_{3}$ are shorter than $\pi$. To see that such triangle is $\left(x_{1}, x_{2}\right)$-angle-deformable, label the midpoint of the edge $x_{2} x_{3}$ by $x_{4}$, so that the segment $x_{1} x_{4}$ splits $T$ into the triangles $T^{\prime}$ with vertices $\left(x_{1}, x_{2}, x_{4}\right)$ and angles $\pi\left(1, \vartheta_{2}, \vartheta_{2}\right)$ and $T^{\prime \prime}$ with vertices $\left(x_{1}, x_{4}, x_{3}\right)$ and angles $\pi\left(1, \vartheta_{3}, \vartheta_{3}\right)$. Also, the edge $x_{1} x_{4}$ has length $\pi \varepsilon / 2$. By Proposition 3.17 the triangle $T^{\prime}$ is $\left(x_{1}, x_{2}\right)$-angle-deformable and so there exists a neighbourhood $\mathcal{N}^{\prime} \subset \mathbb{R}^{2}$ of $\left(\vartheta_{1}, \vartheta_{2}\right)$, a continuous $\theta_{4}: \mathcal{N}^{\prime} \rightarrow \mathbb{R}$ with $\theta_{4}\left(\vartheta_{1}, \vartheta_{2}\right)=\vartheta_{2}$ and a continuous family of triangles $\mathcal{N}^{\prime} \ni \boldsymbol{\nu} \rightarrow T_{\nu}^{\prime}$ such that $T_{\left(\vartheta_{1}, \vartheta_{2}\right)}^{\prime}=T^{\prime}$ and $T_{\boldsymbol{\nu}}$ has angles $\pi\left(\nu_{1}, \nu_{2}, \theta_{4}\left(\nu_{1}, \nu_{2}\right)\right)$. Clearly, the length $\ell_{\boldsymbol{\nu}}$ of the edge $x_{1} x_{4}$ of $T_{\boldsymbol{\nu}}^{\prime}$ depends continuously on $\boldsymbol{\nu}$. Consider now the continuous family $\boldsymbol{\nu} \mapsto T_{\nu}^{\prime \prime}$ of ordinary bigons with opposite vertices $\left(x_{3}, x_{4}^{\prime}\right)$ and angles $\pi\left(\theta_{4}(\boldsymbol{\nu}), 1-\theta_{4}(\boldsymbol{\nu})\right)$ and label by $x_{1}^{\prime}$ a point of the edge $x_{3} x_{4}^{\prime}$ that sits at distance $\ell_{\boldsymbol{\nu}}$ from $x_{4}^{\prime}$. Gluing $T_{\nu}^{\prime}$ and $T_{\nu}^{\prime \prime}$ along the segments $x_{1} x_{4}$ and $x_{1}^{\prime} x_{4}^{\prime}$, we obtain the wished family of triangles parametrized by $\mathcal{N}^{\prime}$.

Case (b): $\vartheta_{2} \in(0,1)$ and $\vartheta_{1} \in(1,2)$.

We proceed as in the proof of Proposition 3.17, case (b). Pick $\eta>0$ and smaller than $\varepsilon, 2(2-$ $\left.\vartheta_{1}\right), 2 \vartheta_{2}$. Then the triple $\left(2-\vartheta_{1}, \vartheta_{2}, 1-\left(\vartheta_{3}+\eta\right)\right)$ satisfies the triangular inequality and so there exists a convex triangle $T^{\prime}$ with vertices $x_{1}, y_{2}, x_{3}$ and angles $\pi\left(\vartheta_{1}-1,1-\vartheta_{2}, \vartheta_{3}+\eta\right)$. Moreover, $\eta$ can be chosen small enough so that such $T^{\prime}$ is $\pi(1-\varepsilon)$-wide at $x_{3}$. Clearly, the edge $y_{2} x_{1}$ of $T^{\prime}$ is shorter than $\pi$. The desired triangle $T$ is then obtained by gluing an ordinary bigon $B_{\vartheta_{2}}$ with vertices $x_{2}$ and $y_{2}^{\prime}$ to $T^{\prime}$ by identifying $y_{2}$ to $y_{2}^{\prime}$ and $y_{2} x_{1}$ to a portion of an edge of $B_{\vartheta_{2}}$. We underline that both $x_{1} x_{2}$ and $x_{1} x_{3}$ are shorter than $\pi$. The double of such a triangle has non-coaxial holonomy and so the triangle is angle-deformable, and in particular $\left(x_{1}, x_{2}\right)$-angle-deformable.

Case $(c): \vartheta_{2} \in(0,1)$ and $\vartheta_{1} \in(2,3)$.

Pick $\eta<0$ such that $|\eta|$ is smaller than $\varepsilon, 2\left(1-\vartheta_{2}\right), \vartheta_{1}-2$. Then the triple $\left(3-\vartheta_{1}, 1-\vartheta_{2}, 1-\left(\vartheta_{3}+\eta\right)\right)$ satisfies the triangular inequality and so there exists a convex triangle $T^{\prime}$ with vertices $x_{1}, y_{2}, x_{3}$ and angles $\pi\left(\vartheta_{1}-2, \vartheta_{2}, \vartheta_{3}+\eta\right)$. Moreover, $\eta$ can be chosen small enough so that such $T^{\prime}$ is $\pi(1-\varepsilon)$-wide at $x_{3}$. Clearly, the edge $x_{1} y_{2}$ of $T^{\prime}$ has length $\ell<\pi$. Consider now a triangle $T^{\prime \prime}=T\left(1, \ell, \vartheta_{2}\right)$ with vertices $x_{2}$ of angle $\pi \vartheta_{2}, x_{2}^{\prime}$ of angle $\pi\left(1-\vartheta_{2}\right)$ and $x_{1}^{\prime}$ of angle $2 \pi$ and edges incident at $x_{1}^{\prime}$ of length 
$l$. The desired triangle $T$ is then obtained by gluing $T^{\prime \prime}$ with $T^{\prime}$ by identifying the edge $x_{1}^{\prime} x_{2}^{\prime}$ of the former to the edge $x_{1} y_{2}$ of the latter. As in the previous case, $x_{1} x_{2}$ and $x_{1} x_{3}$ are shorter than $\pi$ and the triangle is angle-deformable, and in particular $\left(x_{1}, x_{2}\right)$-angle-deformable.

Case (d): $\vartheta_{2}$ not an integer.

Let $d_{1}, d_{3}$ be positive integers such that $\vartheta_{2}^{\prime}=\vartheta_{2}-d_{3} \in(0,1)$ and $\vartheta_{3}^{\prime}=\vartheta_{3}-d_{1} \in(0,2)$ and so $\vartheta_{1}^{\prime}=\vartheta_{1}-d_{1}-d_{3} \in(1,3)$. The previous cases ensure that there exists an $\left(x_{1}, x_{2}\right)$-angledeformable triangle $T^{\prime}$ with angles $\pi\left(\vartheta_{1}^{\prime}, \vartheta_{2}^{\prime}, \vartheta_{3}^{\prime}+\eta\right)$ for some $|\eta|<\varepsilon$, which is $(\pi-\varepsilon)$-wide at $x_{3}$ and such that $\left|x_{1} x_{2}\right|<\pi$ and $\left|x_{1} x_{3}\right|<\pi$. The triangle $T$ is then obtained by gluing an edge of the exceptional bigon $B\left(d_{1},\left|x_{1} x_{3}\right|\right)$ with $x_{1} x_{3}$ and an edge of $B\left(d_{3},\left|x_{1} x_{2}\right|\right)$ with $x_{1} x_{2}$. Such a $T$ is clearly $\pi(1-\varepsilon)$-wide at $x_{3}$. Since this gluing procedure can be performed in families, the $\left(x_{1}, x_{2}\right)$-angle-deformability of $T$ follows from the analogous property of $T^{\prime}$.

Remark 3.19. The restriction $\vartheta_{2} \notin \mathbb{Z}$ is not due to the chosen proof. In fact, for $\vartheta_{2}=1$ and $\vartheta_{1} \notin \mathbb{Z}$, we would be looking for a bigon with different angles which are not multiples of $\pi$ and it is known that such bigons do not exists. As another example, if $\vartheta_{2}=d, \vartheta_{3}=d^{\prime}$ and $\vartheta_{1}=d+d^{\prime}+2$, the double $D T$ a triangle $T$ would be a (connected) ramified cover of $\mathbb{S}^{2}$ over 3 points and this is clearly impossible, as the product of a $d$-cycle and a $d^{\prime}$-cycle in a group of permutations cannot give a $\left(d+d^{\prime}+2\right)$-cycle.

\section{$3.2 \quad$ Cut-and-paste operations}

We recall that, for every $\alpha>0$, we denoted by $S_{\alpha}$ the spherical surface homeomorphic to $\mathbb{S}^{2}$ with two cone points of angle $2 \pi \alpha$ sitting at distance $\pi$, as in Corollary 3.5(a).

\subsubsection{Cut-and-paste at a conical point}

The goal of this section is to describe a cut-and-paste procedure that permits to increase the number of conical points on a sphere by modifying the metric in a neighbourhood of a conical point.

We remind that, if $S$ is a closed spherical surface which is $r$-wide at a conical point $y$, then $U_{y}(r)$ denotes the closed subsurface of $S$ obtained by removing the closed ball of radius $r$ centered at $y$. Thus, $\partial U_{y}(r)$ is a circle of length $2 \pi \alpha \sin (r)$, where $2 \pi \alpha$ is the angle at $y$.

The following lemma is obvious: the situation is illustrated in Figure 10

Lemma 3.20 (Surgery at conical points). Let $S$ and $S^{\prime}$ be two spherical surfaces with cone points $y \in S$ and $y^{\prime} \in S^{\prime}$ of angles $2 \pi \alpha$. Suppose that $S$ is $r$-wide at $y$ and $S^{\prime}$ is $(\pi-r)$-wide at $y^{\prime}$ for some $r \in(0, \pi)$. Then the surface $S \#_{r} S^{\prime}$ obtained by gluing $U=U_{y}(r)$ and $U^{\prime}=U_{y^{\prime}}(\pi-r)$ through an isometry $\partial U \cong \partial U^{\prime}$ is a spherical surface with conical points. Moreover, if $S$ or $S^{\prime}$ has non-coaxial holonomy, the same holds for $S \#_{r} S^{\prime}$.
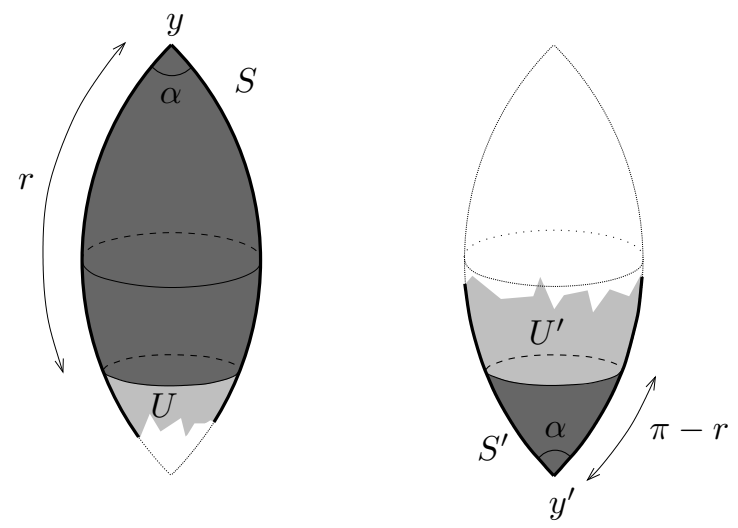

Figure 10: Chopping off the dark region and gluing $U$ and $U^{\prime}$. 


\subsubsection{Cut-and-paste along a path}

We recall that a path $\gamma$ on a surface $S$ is called simple if it is injective (i.e. if its image has no self-intersections).

Definition 3.21. A path $\gamma$ on a spherical surface $S$ is simply developable if its developing map $\operatorname{dev}_{\gamma}$ is injective.

The following lemma is obvious.

Lemma 3.22 (Surgery along a path I). Let $S$ and $S^{\prime}$ be two spherical surfaces. Let $\gamma$ (resp. $\gamma^{\prime}$ ) be a simple path on $S$ (resp. $S^{\prime}$ ) running from the conical point $y_{1}$ of angle $2 \pi \alpha_{1}$ to the conical point $y_{2}$ of angle $2 \pi \alpha_{2}$ (resp. from the conical point $y_{1}^{\prime}$ of angle $2 \pi \alpha_{1}^{\prime}$ to the conical point $y_{2}^{\prime}$ of angle $2 \pi \alpha_{2}^{\prime}$ ) and intersecting the singularities nowhere else. Suppose that $\gamma$ and $\gamma^{\prime}$ are isometric. Then the surface denoted by $S_{\gamma} \# \gamma^{\prime} S^{\prime}$ and obtained by gluing $S \backslash \gamma$ and $S^{\prime} \backslash \gamma^{\prime}$ via the isometric identification of $\gamma$ with $\gamma^{\prime}$ is a spherical surface; moreover, the two points $y_{i}$ and $y_{i}^{\prime}$ are identified to a conical point of angle $2 \pi\left(\alpha_{i}+\alpha_{i}^{\prime}\right)$ on $S_{\gamma} \#_{\gamma^{\prime}} S^{\prime}$ for $i=1,2$.

Using this lemma we get the following result. The situation is illustrated in Figure 11 .

Proposition 3.23 (Surgery along a path II). Consider a spherical surface $S$ with conical points $y_{1}, \ldots, y_{k}$ of angles $2 \pi \beta_{1}, \ldots, 2 \pi \beta_{k}$ and let $\gamma$ be a simple and simply developable path on $S$ that joins $y_{1}$ and $y_{2}$. Let also $d \in \mathbb{Z}_{+}$.

(a) The spherical surface obtained by gluing $S \backslash \gamma$ and $d$ copies of $\mathbb{S}^{2} \backslash \operatorname{dev}_{\gamma}$ via an isometric identification of their boundaries has conical singularities $z_{1}, \ldots, z_{k}$ of angles $2 \pi\left(\beta_{1}+d, \beta_{2}+\right.$ $\left.d, \beta_{3}, \ldots, \beta_{k}\right)$.

(b) Suppose $\beta_{2}<1$ and that $\gamma$ is geodesic path of length $\ell=|\gamma|<\pi$. Then there exists a spherical surface $S^{\prime}$ and a path $\gamma^{\prime}$ on $S^{\prime}$ isometric to $\gamma$ such that $S_{\gamma} \# \gamma^{\prime} S^{\prime}$ has conical singularities $z_{1}, \ldots, z_{k}$ of angles $2 \pi\left(\beta_{1}+2 d, \beta_{2}, \ldots, \beta_{k}\right)$. Moreover, the conical points $z_{1}$ and $z_{2}$ on $S_{\gamma} \# \gamma^{\prime} S^{\prime}$ are joined by a geodesic arc of length $\ell$.

Moreover, if $S$ is deformable (resp. non-coaxial), so are the constructed surfaces.
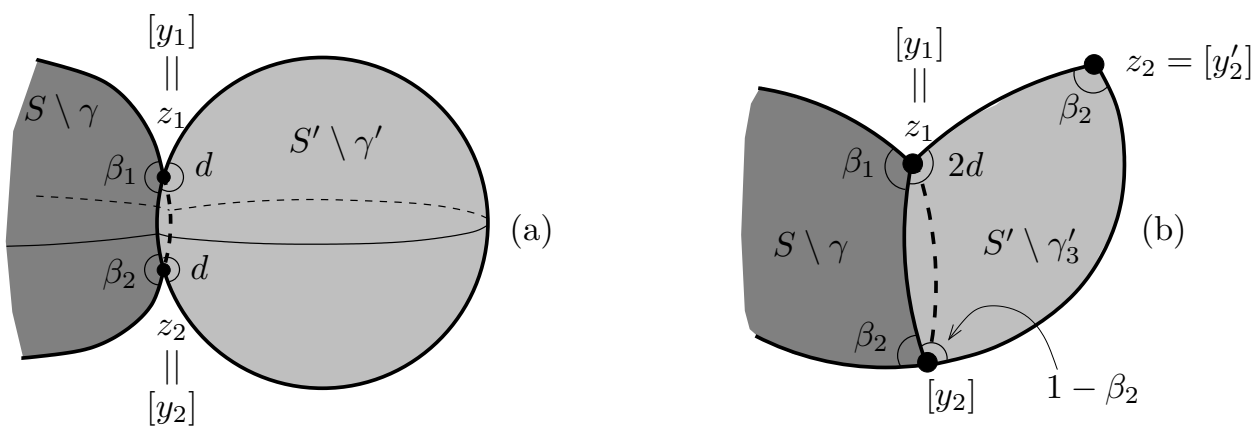

Figure 11: Increasing the angles by $d\left(\boldsymbol{e}_{1}+\boldsymbol{e}_{2}\right)$ and by $2 d \boldsymbol{e}_{1}$.

Proof of Proposition 3.23. About (a), according to Lemma 3.22 it is sufficient to construct a spherical surface $S^{\prime}$ with two conical points of angles $2 \pi d$ that are joined by a simple curve $\gamma^{\prime}$ isometric to $\gamma$. The conical points on the new spherical surface $S_{\gamma} \# \gamma_{\gamma^{\prime}} S^{\prime}$ will be the classes of the $y_{i}$, which will be denoted by $z_{i}$.

To do this, consider the developing map $\operatorname{dev}_{\gamma}$ to $\mathbb{S}^{2}$, which is injective, and call $y_{1}^{\prime}$ and $y_{2}^{\prime}$ its starting and end points. Then let $S^{\prime}$ be the ramified cover of $\mathbb{S}^{2}$ of degree $d$ branched at $y_{1}^{\prime}, y_{2}^{\prime}$ and let $\gamma^{\prime}$ be one of the $d$ lifts of $\operatorname{dev}_{\gamma}$. Indeed, such an $S^{\prime}$ is obtained by gluing $d$ copies of $\mathbb{S}^{2} \backslash \operatorname{dev}_{\gamma}$.

Observe that the path $\gamma$ is still simply developable for any other spherical metric on $S$ sufficiently close to the given one. Thus, if $S$ is deformable, so is the constructed $S_{\gamma} \# \gamma_{\gamma^{\prime}} S^{\prime}$.

Concerning (b), let $S^{\prime}$ be the double of the spherical triangle $T\left(d, \ell, \beta_{2}\right)$ constructed in Lemma 3.11. This is a sphere with conical points $y_{1}^{\prime}, y_{2}^{\prime}, y_{3}^{\prime}$ of angles $2 \pi(2 d), 2 \pi \beta_{2}$ and $2 \pi\left(1-\beta_{2}\right)$ and $y_{1}^{\prime}$ is joined to $y_{2}^{\prime}$ and $y_{3}^{\prime}$ by two geodesics of length $\ell=|\gamma|$ : call them $\gamma_{2}^{\prime}$ and $\gamma_{3}^{\prime}$. The new spherical surface is obtained by gluing $S \backslash \gamma$ to $S^{\prime} \backslash \gamma_{3}^{\prime}$ by identifying $\gamma$ to $\gamma_{3}^{\prime}, y_{1}$ to $y_{1}^{\prime}$ and $y_{2}$ to $y_{3}^{\prime}$. Observe 
that $y_{1}$ and $y_{1}^{\prime}$ merge to a conical point $z_{1}$ of angle $2 \pi\left(\beta_{1}+2 d\right)$, but $y_{2}$ and $y_{3}^{\prime}$ merge to a regular point (i.e. a point of angle $2 \pi$ ). On the other hand, the points $y_{2}^{\prime}, y_{3}, \ldots, y_{k}$ will give rise to conical points $z_{2}, \ldots, z_{k}$ of angles $2 \pi \beta_{2}, \ldots, 2 \pi \beta_{k}$. Finally, notice that $\gamma_{2}^{\prime}$ descends on $S_{\gamma} \# \gamma_{3}^{\prime} S^{\prime}$ to a geodesic path of length $\ell$ between $z_{1}$ and $z_{2}$.

Observe that, for any spherical metric on $S$ sufficiently close to the given one, the path $\gamma$ continuously deforms to a geodesic between $y_{1}$ and $y_{2}$ of length less than $\pi$. Thus, if $S$ is deformable, so is the constructed $S_{\gamma} \# \gamma_{\gamma^{\prime}} S^{\prime}$.

Moreover, considering $S \backslash \gamma$ inside $S_{\gamma} \#{ }_{\gamma^{\prime}} S^{\prime}$, it is easy to see that in both cases (a) and (b) the spherical surface $S_{\gamma} \# \gamma_{\gamma^{\prime}} S^{\prime}$ has the same holonomy as $S$, and so it is non-coaxial if and only if $S$ is.

\subsection{Spheres with four conical points}

In this section we will prove Theorem $\mathrm{C}$ for spheres with four conical points of angles not divisible by $2 \pi$.

Theorem 3.24 (Existence of 4-punctured spherical metrics with non-integral angles). Let $\vartheta_{1}, \vartheta_{2}, \vartheta_{3}, \vartheta_{4}$ be real non-integer numbers that satisfy both the positivity constraints $(P)$ and the holonomy constraints $(\underline{H})$ strictly. Then there exists a sphere $S$ endowed with a spherical metric with 4 conical singularities of angles $2 \pi \vartheta_{1}, \ldots, 2 \pi \vartheta_{4}$ and non-coaxial holonomy.

Remark 3.25. By Luo's Theorem 2.9, all metrics from Theorem 3.24 are deformable.

The proof proceeds in two steps. First we study several types of spherical quadrilaterals embedded and immersed in $\mathbb{S}^{2}$. We construct embedded quadrilaterals that have at most two angles larger than $\pi$ and immersed quadrilaterals with three angles less that $\pi$ and one angle in the interval $(2 \pi, 3 \pi)$. By doubling such quadrilaterals we obtain all spherical metrics with non-integral angles $2 \pi \cdot\left(\vartheta_{1}, \vartheta_{2}, \vartheta_{3}, \vartheta_{4}\right)$ with $\boldsymbol{\vartheta} \in(0,2)^{2} \times(0,1)^{2}$ or $\boldsymbol{\vartheta} \in(2,3) \times(0,1)^{3}$, apart from metrics with two exceptional one parameter families of angles. Spherical surfaces with four conical points in the exceptional classes are obtained by an alternative construction. Finally, using cut-and-paste operations along paths we get all the remaining metrics.

Let $\mathfrak{S}_{4}$ be the group of permutations of $\{1,2,3,4\}$ and view it as acting on $\mathbb{R}^{4}$ in the obvious way $\mathfrak{S}_{4} \times \mathbb{R}^{4} \ni(\sigma, \boldsymbol{\vartheta}) \mapsto \boldsymbol{\vartheta}_{\sigma} \in \mathbb{R}^{4}$, where $\boldsymbol{\vartheta}_{\sigma}:=\left(\vartheta_{\sigma(1)}, \vartheta_{\sigma(2)}, \vartheta_{\sigma(3)}, \vartheta_{\sigma(4)}\right)$. Let $\mathfrak{D}_{8}$ be the subgroup of $\mathfrak{S}_{4}$ generated by (1234) and (13), which is isomorphic to a dihedral group of order 8 . The following simple observation will be useful.

Notation. The four vertices of a quadrilateral $Q$ are always cyclically labelled respecting an orientation on $\partial Q$.

Lemma 3.26 (Allowed permutations). Suppose that there exists a spherical quadrilateral $Q$ with vertices $x_{1}, \ldots, x_{4}$ and conical points of angles $\pi \cdot \boldsymbol{\vartheta}$. Then for every $\sigma \in \mathfrak{D}_{8}$ there exists a spherical quadrilateral $Q^{\prime}$ with vertices $x_{1}^{\prime}, \ldots, x_{4}^{\prime}$ and angles $\pi \cdot \boldsymbol{\vartheta}_{\sigma}$.

Proof. We can produce $Q^{\prime}$ out of $Q$ just cyclically permuting the labels or switching the orientation. In the former case we easily see that this corresponds to the permutation $\sigma_{1}=(1234)$ or $\sigma_{1}=(4321)$; in the latter case, this corresponds to one of the following $\sigma_{2}=(12)(34), \sigma_{2}=(13)(24)$ or $\sigma_{2}=$ (14)(23). Since $\left\{\sigma_{1}, \sigma_{2}\right\}$ generates the $\mathfrak{D}_{8}$, the conclusion follows.

Remark 3.27. Given a surface $S$ of genus 0 with 4 conical points of angles $2 \pi \cdot \boldsymbol{\vartheta}$, we can clearly produce an $S^{\prime}$ with angles $2 \pi \cdot \boldsymbol{\vartheta}_{\sigma}$ for every $\sigma \in \mathfrak{S}_{4}$ : indeed, it is enough to relabel the conical points accordingly to $\sigma$. On the other hand, given a spherical quadrilateral $Q$ with angles $\pi \cdot \boldsymbol{\vartheta}$, it is not always possible to produce a quadrilateral $Q^{\prime}$ with angles $\pi \cdot \boldsymbol{\vartheta}_{\sigma}$ with $\sigma \in \mathfrak{S}_{4}$ but $\sigma \notin \mathfrak{D}_{8}$.

\subsubsection{Convex quadrilaterals}

Let $\boldsymbol{c}=\left(c_{1}, c_{2}, c_{3}, c_{4}\right) \in \mathbb{R}^{4}$ be a vector with strictly half-integral coordinates. Recall that we denote by $\square_{c}$ the unit cube in $\mathbb{R}^{4}$ with centre $\boldsymbol{c}$ and by $\diamond_{\boldsymbol{c}}$ the corresponding truncated cube. Notice that, since $n=4$ is even, $\boldsymbol{m} \in \mathbb{Z}_{o}^{4}$ if and only if $\boldsymbol{m}-\mathbf{1} \in \mathbb{Z}_{o}^{4}$ : thus, $\boldsymbol{\vartheta} \in \mathcal{H}^{4}$ if and only if $\boldsymbol{\delta}=\boldsymbol{\vartheta}-\mathbf{1} \in \mathcal{H}^{4}$. 
Definition 3.28. Let $\boldsymbol{c} \in \mathbb{R}^{4}$ be a strictly half-integral vector and let $\boldsymbol{m}$ be an even integral vertex of $\square_{\boldsymbol{c}}$. The half truncated cube centered at $\boldsymbol{c}$ associated to the vertex $\boldsymbol{m}$ is

$$
\triangle_{\boldsymbol{c}}(\boldsymbol{m}):=\left\{\boldsymbol{p} \in \diamond_{\boldsymbol{c}} \mid d_{1}(\boldsymbol{m}, \boldsymbol{p}) \leq 2\right\} .
$$

Example 3.29. Let $\boldsymbol{c}_{\mathbf{0}}=\left(\frac{1}{2}, \frac{1}{2}, \frac{1}{2}, \frac{1}{2}\right)$ and let $\mathbf{1}=(1,1,1,1)$ and $\mathbf{0}=(0,0,0,0)$ be even vertices of $\square_{c_{0}}$. The truncated cube $\diamond_{c_{0}}$ is the union of $\triangle_{c_{0}}(\mathbf{1})$ and $\triangle_{c_{0}}(\mathbf{0})$; moreover, the two half truncated cubes only overlap along a face.

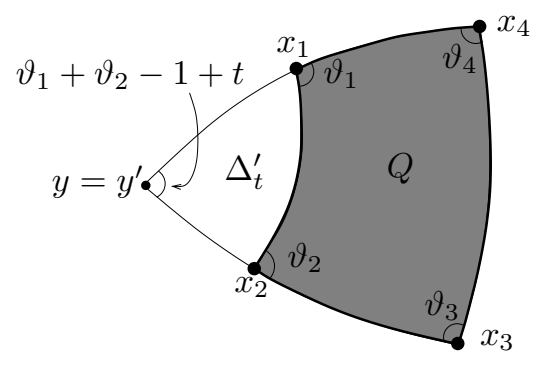

Figure 12: Construction of a convex quadrilateral.

Lemma 3.30 (Convex quadrilaterals). For every $\boldsymbol{\vartheta}=\left(\vartheta_{1}, \ldots, \vartheta_{4}\right)$ in the interior of $\triangle_{\mathbf{c}_{\mathbf{0}}}(\mathbf{1})$ there exists a convex quadrilateral $Q$ with angles $\pi \cdot \boldsymbol{\vartheta}$.

Proof. After possibly reversing the order and cyclically permuting $\vartheta_{1}, \ldots, \vartheta_{4}$, we can assume that $\vartheta_{1} \geq \vartheta_{2}, \vartheta_{3}, \vartheta_{4}$ and $\vartheta_{2} \geq \vartheta_{4}$. In this case one can check that the triple $\left(\vartheta_{1}+\vartheta_{2}-1, \vartheta_{3}, \vartheta_{4}\right)$ satisfies strictly both constraints $(\mathrm{P})$ and $(\mathrm{H})$. Hence, for $t \geq 0$ small enough, there exists a continuous family of spherical triangles $t \mapsto \Delta_{t}$ with vertices $y, x_{3}, x_{4}$ and angles $\pi\left(\vartheta_{1}+\vartheta_{2}-1+t, \vartheta_{3}, \vartheta_{4}\right)$. For $t>0$ small enough there exists as well a continuous family $t \mapsto \Delta_{t}^{\prime}$ of spherical triangles with vertices $y^{\prime}, x_{2}, x_{1}$ and angles $\pi\left(\vartheta_{1}+\vartheta_{2}-1+t, 1-\vartheta_{2}, 1-\vartheta_{1}\right)$. Notice that the diameter of $\Delta_{t}^{\prime} \rightarrow 0$ as $t \rightarrow 0$. Thus, for $t>0$ small enough, it is possible to inscribe $\Delta_{t}^{\prime}$ inside $\Delta_{t}$ in such a way that $y^{\prime}$ coincides with $y$ and that $y^{\prime} x_{2}$ and $y^{\prime} x_{1}$ are contained inside $y x_{3}$ and $y x_{4}$ respectively (see Figure 12). Hence, for such small $t>0$, we can obtain our desired quadrilateral with vertices $x_{1}, x_{2}, x_{3}, x_{4}$ as the completion of $\Delta_{t} \backslash \Delta_{t}^{\prime}$.

\subsubsection{Non-convex quadrilaterals embedded in $\mathbb{S}^{2}$}

Lemma 3.31 (Seven basic non-convex quadrilaterals). Let $\boldsymbol{\vartheta} \in \triangle_{c_{0}}(\mathbf{1})$ and consider the following table.

\begin{tabular}{|c|c|c|c|}
\hline$i$ & $f_{i}(\boldsymbol{\vartheta})$ & $\boldsymbol{m}_{\boldsymbol{i}}$ & $\boldsymbol{c}_{\boldsymbol{i}}$ \\
\hline 0 & $\left(\vartheta_{1}, \vartheta_{2}, \vartheta_{3}, \vartheta_{4}\right)$ & $(1,1,1,1)$ & $\left(\frac{1}{2}, \frac{1}{2}, \frac{1}{2}, \frac{1}{2}\right)$ \\
1 & $\left(2-\vartheta_{1}, 1-\vartheta_{2}, \vartheta_{3}, 1-\vartheta_{4}\right)$ & $(1,0,1,0)$ & $\left(\frac{3}{2}, \frac{1}{2}, \frac{1}{2}, \frac{1}{2}\right)$ \\
2 & $\left(\vartheta_{1}+1,1-\vartheta_{2}, \vartheta_{3}, \vartheta_{4}\right)$ & $(2,0,1,1)$ & $\left(\frac{3}{2}, \frac{1}{2}, \frac{1}{2}, \frac{1}{2}\right)$ \\
3 & $\left(\vartheta_{1}+1, \vartheta_{2}, \vartheta_{3}, \vartheta_{4}+1\right)$ & $(2,1,1,2)$ & $\left(\frac{3}{2}, \frac{1}{2}, \frac{1}{2}, \frac{3}{2}\right)$ \\
4 & $\left(2-\vartheta_{1}, 2-\vartheta_{4}, 1-\vartheta_{3}, 1-\vartheta_{2}\right)$ & $(1,1,0,0)$ & $\left(\frac{3}{2}, \frac{3}{2}, \frac{1}{2}, \frac{1}{2}\right)$ \\
5 & $\left(2-\vartheta_{1}, \vartheta_{4}, 2-\vartheta_{3}, \vartheta_{2}\right)$ & $(1,1,1,1)$ & $\left(\frac{3}{2}, \frac{1}{2}, \frac{3}{2}, \frac{1}{2}\right)$ \\
6 & $\left(1+\vartheta_{1}, 1-\vartheta_{2}, 1+\vartheta_{3}, 1-\vartheta_{4}\right)$ & $(2,0,2,0)$ & $\left(\frac{3}{2}, \frac{1}{2}, \frac{3}{2}, \frac{1}{2}\right)$ \\
7 & $\left(1+\vartheta_{1}, 1-\vartheta_{2}, 2-\vartheta_{3}, \vartheta_{4}\right)$ & $(2,0,1,1)$ & $\left(\frac{3}{2}, \frac{1}{2}, \frac{3}{2}, \frac{1}{2}\right)$ \\
\hline
\end{tabular}

For every convex quadrilateral $Q$ with cyclically ordered angles $\pi \cdot \boldsymbol{\vartheta}$ and for every $1 \leq i \leq 7$ there exists a quadrilateral $Q_{i}$ embedded in $\mathbb{S}^{2}$ with cyclically ordered angles $\pi \cdot f_{i}(\boldsymbol{\vartheta})$. Moreover, $f_{i}$ takes $\mathbf{1}$ to $\boldsymbol{m}_{\boldsymbol{i}}$ and $\triangle_{\boldsymbol{c}_{\mathbf{0}}}(\mathbf{1})$ to $\triangle_{\boldsymbol{c}_{\boldsymbol{i}}}\left(\boldsymbol{m}_{\boldsymbol{i}}\right)$ through an affine map.

Proof. Let us assume that $Q$ is embedded in $\mathbb{S}^{2}$ and that $y_{i}$ is the vertex of $Q$ with angle $\pi \vartheta_{i}$. Denote by $y_{i}^{\prime}$ the point of $\mathbb{S}^{2}$ antipodal to $y_{i}$. The four sides of $Q$ lie on four geodesics in $\mathbb{S}^{2}$ that cut $\mathbb{S}^{2}$ in six convex quadrilaterals and eight convex triangles. All the quadrilaterals in this lemma are assembled from these pieces and the vertices of these quadrilaterals are chosen among the points $y_{i}$ and $y_{j}^{\prime}$. 


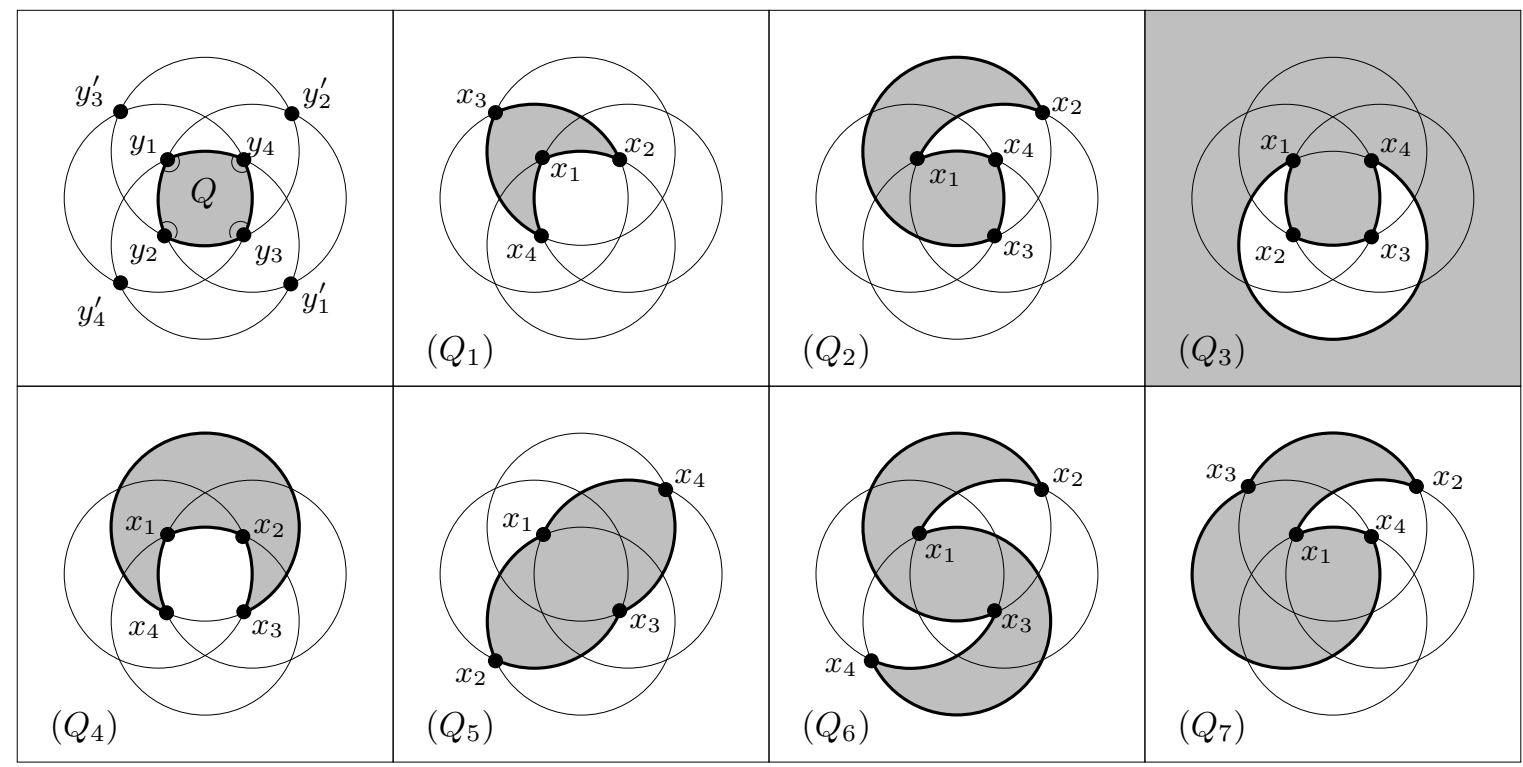

Figure 13: The seven basic non-convex quadrilaterals.

The convex quadrilateral $Q$ and all of seven non-convex quadrilaterals $Q_{i}$ we wish to construct are shown in Figure 13, in all the cases we remove from $\mathbb{S}^{2}$ a point lying in the quadrilateral opposite to $Q$ and we draw the four great circles on which the edges of $Q$ lie.

Remark 3.32. In quadrilaterals $\left(Q_{1}\right)$ and $\left(Q_{2}\right)$ the vertex $x_{1}$ is the only one with angle larger than $\pi$ and both adjacent sides (namely, $x_{1} x_{2}$ and $x_{1} x_{4}$ ) are shorter than $\pi$. In quadrilaterals $\left(Q_{3}\right), \ldots,\left(Q_{7}\right)$ there are two opposite sides shorter than $\pi$ that join a vertex with an angle larger than $\pi$ with a vertex with an angle less than $\pi$.

We will now show that the angles of the quadrilaterals constructed in Lemma 3.31 cover almost all points of $\diamond_{\left(\frac{3}{2}, \frac{1}{2}, \frac{1}{2}, \frac{1}{2}\right)}$ and $\diamond_{\left(\frac{3}{2}, \frac{3}{2}, \frac{1}{2}, \frac{1}{2}\right)}$.

Corollary 3.33 (Non-convex quadrilaterals I). Let $\boldsymbol{\vartheta}$ be in the interior of $\diamond_{\left(\frac{3}{2}, \frac{1}{2}, \frac{1}{2}, \frac{1}{2}\right)}$ but $\boldsymbol{\vartheta} \neq$ $(1+a, 1-a, 1-a, 1-a)$ for all $a \in(0,1)$. Then for some permutation $\sigma \in \mathfrak{S}_{4}$ there exists $a$ spherical quadrilateral with angles $\pi \cdot \boldsymbol{\vartheta}_{\sigma}$.

Proof. To prove this corollary we will use quadrilaterals of type $\left(Q_{1}\right)$ and $\left(Q_{2}\right)$. Consider the set of points in $\diamond_{\left(\frac{3}{2}, \frac{1}{2}, \frac{1}{2}, \frac{1}{2}\right)}$ that can be represented by quadrilaterals of type $\left(Q_{1}\right)$ in Lemma 3.31. From Lemma 3.30 it follows that these points are exactly those at distance less than 2 from the point $\boldsymbol{m}_{\mathbf{1}}=(1,0,1,0)$. In the same way, quadrilaterals of type $\left(Q_{2}\right)$ correspond to points of $\diamond_{\left(\frac{3}{2}, \frac{1}{2}, \frac{1}{2}, \frac{1}{2}\right)}$ at distance less than 2 from the point $\boldsymbol{m}_{\mathbf{2}}=(2,0,1,1)$.

Now, the group of coordinate permutations preserving $\square_{\left(\frac{3}{2}, \frac{1}{2}, \frac{1}{2}, \frac{1}{2}\right)}$ can be identified to $\mathfrak{S}_{3} \cong$ $\operatorname{stab}(1) \subset \mathfrak{S}_{4}$. The union of the orbits of the points $\boldsymbol{m}_{\mathbf{1}}$ and $\boldsymbol{m}_{\mathbf{2}}$ under this group consists of the following six vertices of $\diamond_{\left(\frac{3}{2}, \frac{1}{2}, \frac{1}{2}, \frac{1}{2}\right)}$ :

$$
(1,0,1,0),(1,1,0,0),(1,0,0,1) ; \quad(2,0,1,1),(2,1,0,1),(2,1,1,0) .
$$

Hence six halves of $\nabla_{\left(\frac{3}{2}, \frac{1}{2}, \frac{1}{2}, \frac{1}{2}\right)}$ are covered by $\vartheta$ corresponding to spherical quadrilaterals (see Remark (3.29). It is easy to see that points of type $(1+a, 1-a, 1-a, 1-a)$ are the only points that are not covered. These are exactly the points in $\diamond_{\left(\frac{3}{2}, \frac{1}{2}, \frac{1}{2}, \frac{1}{2}\right)}$ that are on distance 2 from the above six vertices.

Corollary 3.34 (Non-convex quadrilaterals II). Let $\boldsymbol{\vartheta}$ be in the interior of $\diamond_{\left(\frac{3}{2}, \frac{3}{2}, \frac{1}{2}, \frac{1}{2}\right)}$. Then for some permutation $\sigma \in \mathfrak{S}_{4}$ there exists a spherical quadrilateral with angles $\pi \cdot \boldsymbol{\vartheta}_{\sigma}^{2}$.

Proof. The argument is similar to the one employed in the proof of Corollary 3.33 but in this case we use quadrilaterals of types $\left(Q_{3}\right),\left(Q_{4}\right),\left(Q_{5}\right),\left(Q_{6}\right)$ and $\left(Q_{7}\right)$. By Lemma 3.31, after taking coordinate 
permutations, we see that $\boldsymbol{m}_{\mathbf{3}}, \ldots, \boldsymbol{m}_{\mathbf{7}}$ correspond to the eight even vertices of $\diamond_{\left(\frac{3}{2}, \frac{3}{2}, \frac{1}{2}, \frac{1}{2}\right)}$, namely

$$
\begin{array}{ccc}
(2,2,1,1) ; \quad(1,1,0,0) ; & (1,1,1,1) ; \quad(2,2,0,0) ; \\
(2,1,0,1), & (2,1,1,0),(1,2,0,1),(1,2,1,0) .
\end{array}
$$

Thus, the construction of $\left(Q_{3}\right),\left(Q_{4}\right),\left(Q_{5}\right),\left(Q_{6}\right)$ and $\left(Q_{7}\right)$ provides quadrilaterals corresponding to points in $\diamond_{\left(\frac{3}{2}, \frac{3}{2}, \frac{1}{2}, \frac{1}{2}\right)}$ at distance less than 2 from all eight even vertices.

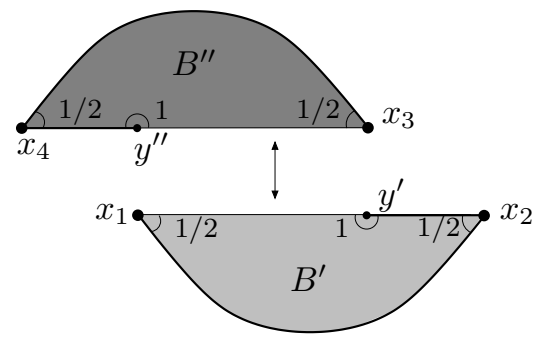

Figure 14: A quadrilateral with angles $\pi \cdot\left(\frac{3}{2}, \frac{1}{2}, \frac{3}{2}, \frac{1}{2}\right)$.

The only point at distance at least 2 from all eight even vertices of $\nabla_{\left(\frac{3}{2}, \frac{3}{2}, \frac{1}{2}, \frac{1}{2}\right)}$ is its center. In order to construct a quadrilateral with angles $\pi \cdot\left(\frac{3}{2}, \frac{1}{2}, \frac{3}{2}, \frac{1}{2}\right)$, consider two ordinary bigons $B^{\prime}$ and $B^{\prime \prime}$ of angle $\pi / 2$, with vertices $x_{1}, x_{2}$ and $x_{3}, x_{4}$. Let $r \in(0, \pi)$ and pick a point $y^{\prime} \in \partial B^{\prime}$ at distance $r$ from $x_{1}$ and a point $y^{\prime \prime} \in \partial B^{\prime \prime}$ at distance $r$ from $x_{3}$. The wished quadrilateral is then obtained by gluing $B^{\prime}$ and $B^{\prime \prime}$ via the isometric identification of $x_{1} y^{\prime}$ with $y^{\prime \prime} x_{3}$ (see Figure 14).

\subsubsection{Quadrilaterals immersed in $\mathbb{S}^{2}$}

In order to construct spheres with four conical points with angles in $\diamond_{\left(\frac{5}{2}, \frac{1}{2}, \frac{1}{2}, \frac{1}{2}\right)}$, we proceed as in the previous section.

Lemma 3.35 (Three basic immersed quadrilaterals). Let $\boldsymbol{\vartheta} \in \triangle_{\boldsymbol{c}_{\mathbf{0}}}(\mathbf{1})$ and consider the following table.

\begin{tabular}{|c|c|c|c|}
\hline$i$ & $f_{i}(\boldsymbol{\vartheta})$ & $\boldsymbol{m}_{\boldsymbol{i}}$ & $\boldsymbol{c}_{\boldsymbol{i}}$ \\
\hline 8 & $\left(3-\vartheta_{1}, 1-\vartheta_{4}, \vartheta_{3}, \vartheta_{2}\right)$ & $(2,0,1,1)$ & $\left(\frac{5}{2}, \frac{1}{2}, \frac{1}{2}, \frac{1}{2}\right)$ \\
9 & $\left(2+\vartheta_{1}, 1-\vartheta_{2}, \vartheta_{3}, 1-\vartheta_{4}\right)$ & $(3,0,1,0)$ & $\left(\frac{5}{2}, \frac{1}{2}, \frac{1}{2}, \frac{1}{2}\right)$ \\
10 & $\left(2+\vartheta_{1}, \vartheta_{2}, \vartheta_{3}, \vartheta_{4}\right)$ & $(3,1,1,1)$ & $\left(\frac{5}{2}, \frac{1}{2}, \frac{1}{2}, \frac{1}{2}\right)$ \\
\hline
\end{tabular}

For every convex quadrilateral $Q$ with cyclically ordered angles $\pi \cdot \vartheta$ and for every $8 \leq i \leq 10$ there exists a quadrilateral $Q_{i}$ with cyclically ordered angles $\pi \cdot f_{i}(\boldsymbol{\vartheta})$. Moreover, $f_{i}$ takes $\mathbf{1}$ to $\boldsymbol{m}_{\boldsymbol{i}}$ and $\triangle_{\boldsymbol{c}_{\mathbf{0}}}(\mathbf{1})$ to $\triangle_{\boldsymbol{c}_{\boldsymbol{i}}}\left(\boldsymbol{m}_{\boldsymbol{i}}\right)$ through an affine map.

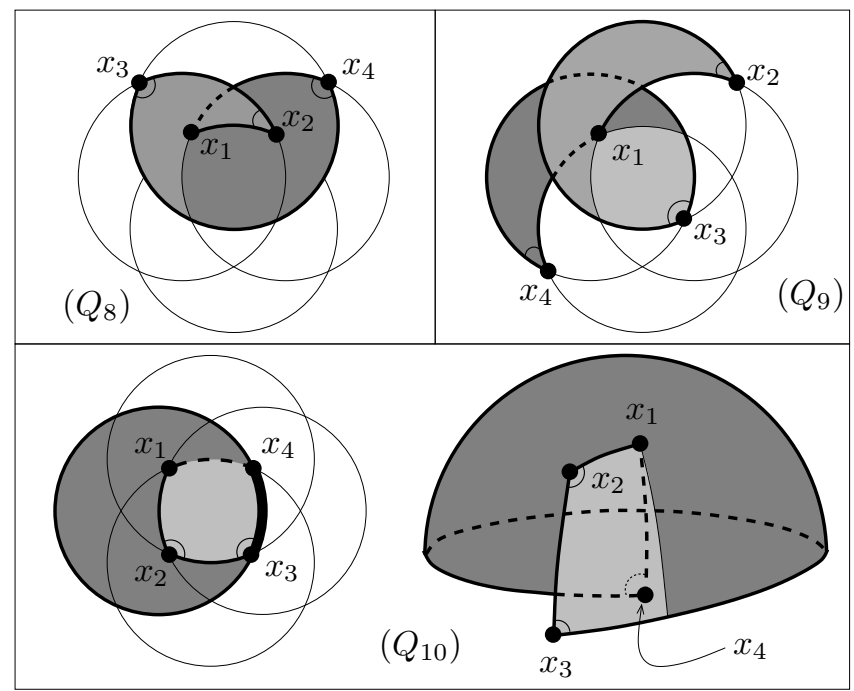


Figure 15: Three families of immersed quadrilaterals.

Proof. The quadrilaterals are illustrated in Figure 15 as immersed in $\mathbb{S}^{2}$, though we have included also another picture of $Q_{10}$ for clarity.

The existence of such quadrilaterals relies on Lemma 3.31. In fact, in order to construct $Q_{8}$ consider the quadrilateral $Q_{1}$ and call $x_{1}^{\prime}, x_{2}^{\prime}, x_{3}^{\prime}, x_{4}^{\prime}$ its vertices and let $B=B_{\vartheta_{2}}$ be an ordinary bigon with vertices $y_{4}$ and $y_{4}^{\prime}$. Call $z$ one of the two points on the boundary of $B$ at distance $\left|x_{4}^{\prime} x_{1}^{\prime}\right|$ from $y_{4}^{\prime}$. The quadrilateral $Q_{8}$ is obtained from $Q_{1}$ and $B$ by gluing the edge $x_{4}^{\prime} x_{1}^{\prime}$ with $y_{4}^{\prime} z$, so that $x_{4}^{\prime}$ and $y_{4}^{\prime}$ are identified to a smooth point (which will not be marked as a vertex of $Q_{8}$ ): its vertices are $x_{1}:=\left[x_{1}^{\prime}\right]=[z], x_{2}:=\left[x_{4}^{\prime}\right], x_{3}:=\left[x_{3}^{\prime}\right]$ and $x_{4}=:\left[y_{4}\right]$.

In a similar fashion, consider $Q$ with vertices $x_{i}^{\prime}$ and let $B^{\prime \prime}=B_{1-\vartheta_{2}}$ and $B^{\prime \prime \prime}=B_{1-\vartheta_{3}}$ be two ordinary bigons with vertices $x^{\prime \prime}, y^{\prime \prime}$ and $x^{\prime \prime \prime}, y^{\prime \prime \prime}$ respectively. Let $z^{\prime \prime}$ be a point on $\partial B^{\prime \prime}$ at distance $\left|x_{2}^{\prime} x_{1}^{\prime}\right|$ from $y^{\prime \prime}$ and let $z^{\prime \prime \prime}$ be a point on $\partial B^{\prime \prime \prime}$ at distance $\left|x_{3}^{\prime} x_{1}^{\prime}\right|$ from $y^{\prime \prime \prime}$. The quadrilateral $Q_{9}$ is then obtained by gluing $x_{2}^{\prime} x_{1}^{\prime}$ to $y^{\prime \prime} z^{\prime \prime}$ and $x_{3}^{\prime} x_{1}^{\prime}$ to $y^{\prime \prime \prime} z^{\prime \prime \prime}$ and calling $x_{1}:=\left[x_{1}^{\prime}\right]=\left[z^{\prime \prime}\right], x_{2}:=\left[x^{\prime \prime}\right]$, $x_{3}:=\left[x_{3}^{\prime}\right], x_{4}:=\left[x^{\prime \prime \prime}\right]$.

Finally, start again from the quadrilateral $Q$ with vertices $x_{i}^{\prime}$ and the triangle $T=T\left(1,\left|x_{1}^{\prime} x_{4}^{\prime}\right|, 1-\right.$ $\left.\vartheta_{4}\right)$ with vertices $y_{1}, y_{3}, y_{4}$ of angles $\pi\left(2,1-\vartheta_{4}, \vartheta_{4}\right)$. The wished $Q_{10}$ is obtained by identifying $y_{1} y_{3}$ to $x_{1}^{\prime} x_{4}^{\prime}$ and then calling $x_{1}:=\left[x_{1}^{\prime}\right]=\left[y_{1}\right], x_{2}:=\left[y_{2}^{\prime}\right], x_{3}:=\left[x_{3}^{\prime}\right]$ and $x_{4}:=\left[y_{4}\right]$.

Corollary 3.36 (Immersed quadrilaterals). Let $\boldsymbol{\vartheta}$ be in the interior of $\diamond_{\left(\frac{5}{2}, \frac{1}{2}, \frac{1}{2}, \frac{1}{2}\right)}$ but $\boldsymbol{\vartheta} \neq(2+$ $a, a, a, a)$ for all $a \in\left(0, \frac{1}{2}\right]$. Then for some permutation $\sigma \in \mathfrak{S}_{4}$ there exists a spherical quadrilateral with angles $\pi \cdot \boldsymbol{\vartheta}_{\sigma}$. Moreover, the vertex with angle larger than $2 \pi$ can be joined to any other vertex with a smooth geodesic of length strictly less than $\pi$.

Proof. The argument is similar to the one employed in the proof of Corollaries 3.33 and 3.34. This time we use quadrilaterals of types $\left(Q_{8}\right),\left(Q_{9}\right),\left(Q_{10}\right)$.

By Lemma 3.31, after taking coordinate permutations, we see that $\boldsymbol{m}_{\mathbf{8}}, \boldsymbol{m}_{\mathbf{9}}, \boldsymbol{m}_{\mathbf{1 0}}$ correspond to the seven even vertices of $\diamond_{\left(\frac{5}{2}, \frac{1}{2}, \frac{1}{2}, \frac{1}{2}\right)}$, namely

$$
(3,1,1,1) ; \quad(2,1,1,0),(2,1,0,1),(2,0,1,1) ; \quad(3,1,0,0),(3,0,1,0),(3,0,0,1) .
$$

Thus, the construction of $\left(Q_{8}\right),\left(Q_{9}\right),\left(Q_{10}\right)$ provides quadrilaterals corresponding to points in $\diamond_{\left(\frac{3}{2}, \frac{3}{2}, \frac{1}{2}, \frac{1}{2}\right)}$ at distance less than 2 from these seven vertices. The remaining points belong to the interval connecting the vertex $(2,0,0,0)$ with the centre of $\diamond_{\left(\frac{5}{2}, \frac{1}{2}, \frac{1}{2}, \frac{1}{2}\right)}$. The last assertion can be checked by direct inspection.

\subsubsection{Sporadic families of 4-punctured spheres}

Though most 4-punctured spheres can be obtained by doubling spherical quadrilaterals, it seems from Lemma 3.33 and Lemma 3.35 that there are two 1-parameter families of $\boldsymbol{\vartheta} \in \mathbb{R}^{4}$ such that we are not able to construct quadrilaterals with angles $\pi \cdot \boldsymbol{\vartheta}$. Thus, for such families of angles, here we present ad-hoc constructions.

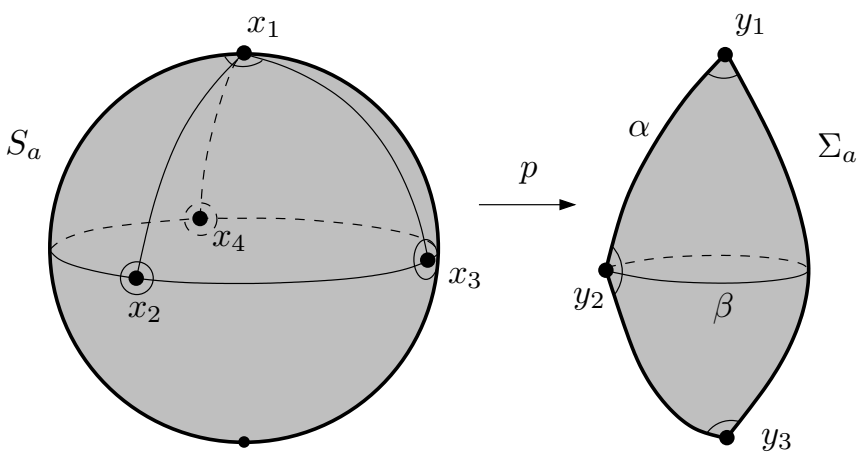

Figure 16: The sporadic sphere $S_{a}$ with the six paths $\gamma_{i j}$. 
Lemma 3.37 (Sporadic 4-punctured spheres). (a) For any $a \in(0,1)$ there exists a 4-punctured sphere $S_{a}$ and a spherical metric on it with conical singularities $x_{1}, x_{2}, x_{3}, x_{4}$ of angles $2 \pi$. $(1+a, 1-a, 1-a, 1-a)$.

(b) For any $b \in\left(0, \frac{1}{2}\right)$ there exists a 4-punctured sphere $S_{b}$ and a spherical metric on it with conical singularities $x_{1}, x_{2}, x_{3}, x_{4}$ of angles $2 \pi \cdot(2+b, b, b, b)$.

(c) There exists a 4-punctured sphere $S$ and a spherical metric on it with conical singularities $x_{1}, x_{2}, x_{3}, x_{4}$ of angles $2 \pi \cdot\left(\frac{5}{2}, \frac{1}{2}, \frac{1}{2}, \frac{1}{2}\right)$.

Moreover, on the spheres constructed in all three cases there is a smooth geodesic from $x_{1}$ to $x_{j}$ of length strictly less than $\pi$ for $j=2,3,4$.

Proof. As for part (a), notice that for every $a \in(0,1)$ there exists a sphere $\Sigma_{a}$ with three conical points $y_{1}, y_{2}, y_{3}$ of angles $2 \pi \cdot \boldsymbol{\vartheta}(a)$, where $\boldsymbol{\vartheta}(a):=\left(\frac{1+a}{3}, 1-a, \frac{1}{3}\right)$. Indeed, $\boldsymbol{\vartheta}(0)=\left(\frac{2}{3}, 1, \frac{1}{3}\right)$ and $\boldsymbol{\vartheta}(1)=$ $\left(\frac{1}{3}, 0, \frac{1}{3}\right)$ lie on the boundary of the simplex formed by the angle vectors $\boldsymbol{\vartheta} \in \mathbb{R}^{3}$ corresponding to spheres with three angles less than $2 \pi$ and $\boldsymbol{\vartheta}(a)$ lies strictly inside such a simplex for $a \in(0,1)$.

Now consider the cyclic cover $p: S_{a} \rightarrow \Sigma_{a}$ of degree three cover branched over $y_{1}$ and $y_{3}$. The $x_{1}=p^{-1}\left(y_{1}\right)$ is a point of angle $2 \pi(1+a)$ and $p^{-1}\left(y_{3}\right)$ is a smooth point, which will not be labelled. Moreover, $p^{-1}\left(y_{2}\right)$ consists of three points of angle $2 \pi(1-a)$, which we label by $x_{2}, x_{3}, x_{4}$ (see Figure (16). Thus, $\left(S_{a}, x_{1}, x_{2}, x_{3}, x_{4}\right)$ is our wished spherical surface. Three geodesics that joint $x_{1}$ with $x_{j}$ are preimages on $S_{a}$ of the shortest geodesic in $\Sigma_{a}$ joining $y_{1}$ and $y_{2}$.

The proof of (b) is entirely analogous. As above, for any $b \in\left(0, \frac{1}{2}\right)$ there exists a sphere $\Sigma_{b}$ with three conical points $y_{1}, y_{2}, y_{3}$ of angles $2 \pi \cdot \boldsymbol{\vartheta}(b)$, where $\boldsymbol{\vartheta}(b):=\left(\frac{2+b}{3}, b, \frac{1}{3}\right)$. Indeed, $\boldsymbol{\vartheta}(0)=\left(\frac{2}{3}, 0, \frac{1}{3}\right)$ and $\boldsymbol{\vartheta}\left(\frac{1}{2}\right)=\left(\frac{5}{6}, \frac{1}{2}, \frac{1}{3}\right)$ lie on the boundary of the simplex formed by the angle vectors $\boldsymbol{\vartheta} \in \mathbb{R}^{3}$ corresponding to spheres with three angles less than $2 \pi$, and $\boldsymbol{\vartheta}(b)$ lies strictly inside it for $b \in\left(0, \frac{1}{2}\right)$. Now, as before take the cyclic cover of degree three $S_{b} \rightarrow \Sigma_{b}$ branched at $y_{1}$ and $y_{3}$.

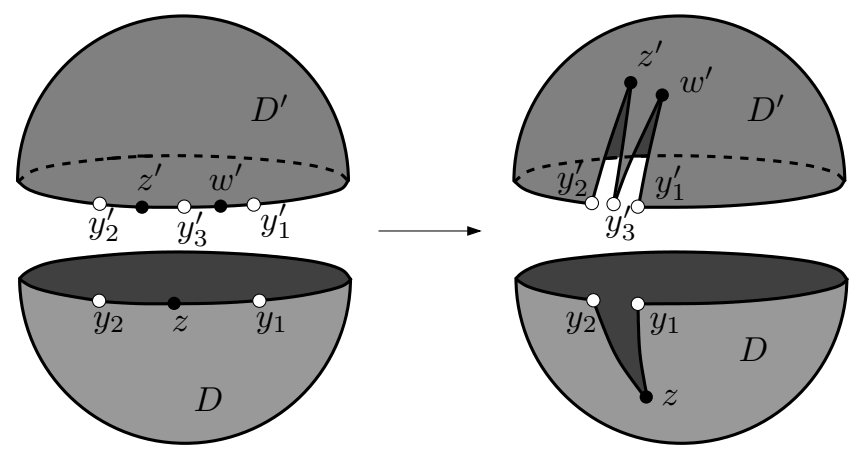

Figure 17: A sphere with angles $2 \pi \cdot\left(\frac{5}{2}, \frac{1}{2}, \frac{1}{2}, \frac{1}{2}\right)$.

About (c), consider two hemispheres $D$ and $D^{\prime}$ and let $\ell \in(0, \pi)$. On $\partial D$ pick points $y_{1}, z, y_{2}$ (in this cyclic order with respect to the orientation induced on $\partial D$ ) in such a way that $\left|y_{1} y_{2}\right|=\ell$ and $z$ is the midpoint of $y_{1} y_{2}$; on $\partial D^{\prime}$ pick points $y_{2}^{\prime}, z^{\prime}, y_{3}^{\prime}, w^{\prime}, y_{1}^{\prime}$ (in this cyclic order) in such a way that $\left|y_{2}^{\prime} y_{1}^{\prime}\right|=\ell$ and that $z^{\prime}$ is the midpoint of $y_{2}^{\prime} y_{3}^{\prime}$ and $w^{\prime}$ is the midpoint of $y_{3}^{\prime} y_{1}^{\prime}$. As in Figure 17. the wished sphere $S$ is obtained by identifying $y_{1} z$ to $z y_{2}$ on $D$ and $y_{2}^{\prime} z^{\prime}$ to $z^{\prime} y_{3}^{\prime}$ and $y_{3}^{\prime} w^{\prime}$ to $w^{\prime} y_{1}^{\prime}$ on $D^{\prime}$, and finally $y_{1}^{\prime} y_{2}^{\prime}$ to $y_{1} y_{2}$ : its marked points are $x_{1}:=\left[y_{i}\right]=\left[y_{i}^{\prime}\right], x_{2}=[z], x_{3}=\left[w^{\prime}\right]$, $x_{4}=\left[z^{\prime}\right]$. The last assertion is clear by construction.

\subsubsection{Spheres with $\vartheta_{1}, \vartheta_{2}<2, \vartheta_{3}, \vartheta_{4}<1$ and with $1<\vartheta_{1}<2, \vartheta_{2}, \vartheta_{3}, \vartheta_{4}<1$}

Here we derive corollaries from the statements proven in the previous sections. Denote by $\Pi^{4}$ the box $[1,2] \times[0,2] \times[0,1]^{2} \subset \mathbb{R}^{4}$.

Proposition 3.38 (4-punctured spheres with non-integral angles in $\left.\Pi^{4}\right)$. For every $\vartheta \in \operatorname{int}\left(\Pi^{4} \cap \mathcal{A}^{4}\right)$ with no integral coordinate there exists a sphere $S$ with a spherical metric $g$ and conical singularities $x_{1}, x_{2}, x_{3}, x_{4}$ of angles $2 \pi \cdot \boldsymbol{\vartheta}$, which satisfies the following properties: 
(a) there exist six simple paths $\left\{\gamma_{i j} \mid 1 \leq i<j \leq 4\right\}$ that have no inner points of intersection and such that $\gamma_{i j}$ joins $x_{i}$ and $x_{j}$;

(b) either $\gamma_{13}$ or $\gamma_{14}$ is a geodesic shorter than $\pi$;

(c) the metric g has non-coaxial holonomy.

Proof. We will first construct the spheres and the paths $\gamma_{i j}$ and then will prove that their holonomy is not coaxial.

Construction of spheres with six paths. Notice that a $\vartheta \in \operatorname{int}\left(\Pi^{4} \cap \mathcal{A}^{4}\right)$ with no integral coordinate must belong to the interior either of $\diamond_{\left(\frac{3}{2}, \frac{1}{2}, \frac{1}{2}, \frac{1}{2}\right)}$ or of $\nabla_{\left(\frac{3}{2}, \frac{3}{2}, \frac{1}{2}, \frac{1}{2}\right)}$. Hence, by doubling quadrilaterals $Q$ constructed in Corollaries 3.33 and 3.34, we cover all the cases apart from the exceptional family treated in Lemma 3.37(a).

In order to find the six paths $\gamma_{i j}$, we proceed as follows.

Consider first the case of $S$ obtained as a double quadrilateral $D Q=Q \sqcup \bar{Q} / \sim$. Take four geodesic paths $\gamma_{12}, \gamma_{23}, \gamma_{34}, \gamma_{14}$ corresponding to the edges of $Q$ (or; equivalently of $\bar{Q}$ ) and choose a simple path $\gamma_{13}$ inside $Q$ and a simple path $\gamma_{24}$ inside $\bar{Q}$. All these paths will be simple, since the quadrilateral is embedded in $\mathbb{S}^{2}$. Moreover it follows from Remark 3.32 that either $\gamma_{13}$ or $\gamma_{14}$ can be chosen to be a geodesic shorter than $\pi$.

Consider now the exceptional spheres $S_{a}$ with $a \in(0,1)$, constructed in Lemma 3.37(a). By Lemma 3.14, the surface $\Sigma_{a}$ can be constructed by doubling of a spherical triangle $T_{a}$ with vertices $y_{1}, y_{2}, y_{3}$ and angles $\pi \cdot\left(\frac{1+a}{3}, 1-a, \frac{1}{3}\right)$. Choose a point $z \in T_{a}$ in the interior of the side $y_{1} y_{3}$. Now consider the following two paths on $\Sigma_{a}=D T_{a}$ : the geodesic $\alpha$ determined by the edge $y_{1} y_{2}$ of $T_{a}$ and the geodesic $\beta$ obtained by doubling of the geodesic segment $y_{2} z$ contained in $T_{a}$. Clearly, $\beta$ is a simple loop on $\Sigma_{a}$ based at $y_{2}$ and it is easy to see that $\alpha$ is shorter than $\pi$. If $p: S_{a} \rightarrow \Sigma_{a}$ is the triple cyclic cover branched at $y_{1}, y_{3}$, then we define $\gamma_{12}, \gamma_{13}, \gamma_{14}$ to be the preimages of $\alpha$ and $\gamma_{23}, \gamma_{34}, \gamma_{24}$ to be the preimages of $\beta$ through $p$. Since $|\alpha|<\pi$, we have that both $\gamma_{13}$ and $\gamma_{14}$ are shorter than $\pi$.

This completes the proof of claims (a) and (b).

Non-coaxiality.

Since $\vartheta_{i} \notin \mathbb{Z}$, non-coaxiality of the holonomy of the spherical surfaces $S$ just constructed follows from Lemma 2.10 if we are able to find two distinct conical points $x_{i}, x_{j}$ on $S$ joined by a smooth geodesic $\gamma$ of length $\ell$ with $\ell \notin \pi \mathbb{Z}$. By the above property (b), we can choose $\gamma$ to be either the path $\gamma_{13}$ or $\gamma_{14}$. This proves (c).

Analogously, we have the following.

Proposition 3.39 (4-punctured spheres with non-integral angles in $\left.\diamond_{\left(\frac{5}{2}, \frac{1}{2}, \frac{1}{2}, \frac{1}{2}\right)}\right)$. F For every $\boldsymbol{\vartheta}$ in the interior of $\bigotimes_{\left(\frac{5}{2}, \frac{1}{2}, \frac{1}{2}, \frac{1}{2}\right)}$ there exists a sphere $S$ with a spherical metric $g$ and conical singularities $x_{1}, x_{2}, x_{3}, x_{4}$ of angles $2 \pi \cdot \boldsymbol{\vartheta}$, which satisfies the following properties:

(a) $x_{1}$ and $x_{2}$ are joined by a smooth geodesic of length strictly less than $\pi$;

(b) the metric g has non-coaxial holonomy.

Proof. The wished spherical surface $S$ is either obtained from Lemma 3.37(b-c) or by doubling the quadrilaterals constructed in Corollary 3.36. In either case, property (a) is satisfied.

Property (b) then follows from Lemma 2.10, since $\vartheta_{1}, \vartheta_{2} \notin \mathbb{Z}$.

\subsubsection{Existence of spheres with 4 conical points and non-integral angles}

In this section we finally prove Theorem 3.24, We will construct the desired spherical surfaces starting from those produced in Proposition 3.38 and applying the gluing operations of Proposition 3.23. Since these surgeries do not change the holonomy, non-coaxiality of the new metrics follows from Proposition 3.38 . 
Notation. Let $\boldsymbol{e}_{1}, \ldots, \boldsymbol{e}_{\mathbf{4}}$ be the standard generators of $\mathbb{Z}^{4}$ and define $\boldsymbol{e}_{\boldsymbol{k} l}:=\boldsymbol{e}_{\boldsymbol{k}}+\boldsymbol{e}_{\boldsymbol{l}}$ for $1 \leq k<$ $l \leq 4$. The six elements $\boldsymbol{e}_{\boldsymbol{k} l}$ generate the semigroup

$$
\Gamma^{4}:=\left\{\boldsymbol{p} \in \mathbb{Z}_{\geq 0}^{4} \mid\|\boldsymbol{p}\|_{1} \in 2 \mathbb{Z} \text { and } 2 p_{j} \leq\|\boldsymbol{p}\|_{1} \text { for all } j=1, \ldots, 4\right\} .
$$

Proof of Theorem 3.24. Let $\boldsymbol{m}$ be a point in $\mathbb{Z}_{\geq 0}^{4}$ such that $\boldsymbol{\vartheta} \in \square_{\boldsymbol{c}}$, where $\boldsymbol{c}=\boldsymbol{m}+\left(\frac{1}{2}, \frac{1}{2}, \frac{1}{2}, \frac{1}{2}\right)$. Without loss of generality, we can assume $m_{1} \geq m_{2} \geq m_{3} \geq m_{4} \geq 0$, and also $m_{1} \geq 1$.

We will now treat two cases separately.

Case (a): $m_{1} \leq m_{2}+m_{3}+m_{4}$.

Suppose first that $\|\boldsymbol{m}\|_{1} \in 2 \mathbb{Z}$ so that $\boldsymbol{m} \in \Gamma^{4}$.

Since $m_{1} \geq 1$, we have $m_{2} \geq 1$ and so $\boldsymbol{m}^{\prime}=\boldsymbol{m}-\boldsymbol{e}_{\mathbf{1 2}} \in \Gamma^{4}$. As a consequence, we have a presentation

$$
\boldsymbol{m}^{\prime}=m_{12} \boldsymbol{e}_{\mathbf{1 2}}+\cdots+m_{34} \boldsymbol{e}_{\mathbf{3 4}}
$$

for suitable $m_{i j} \in \mathbb{Z}_{\geq 0}$ and so $\boldsymbol{\vartheta}-\boldsymbol{m}^{\prime} \in[1,2] \times[1,2] \times[0,1]^{2} \subset \Pi^{4}$. Since $\left\|\boldsymbol{m}^{\prime}\right\| \in 2 \mathbb{Z}$, the vector $\boldsymbol{\vartheta}-\boldsymbol{m}^{\boldsymbol{\prime}} \in \mathcal{A}^{4}$ and so $\boldsymbol{\vartheta}-\boldsymbol{m}^{\boldsymbol{\prime}} \in \operatorname{int}\left(\Pi^{4} \cap \mathcal{A}^{4}\right)$ and it has no integral coordinate.

By Proposition 3.38 there exists a sphere $S^{\prime}$ with four conical points of angles $2 \pi \cdot\left(\boldsymbol{\vartheta}-\boldsymbol{m}^{\prime}\right)$ and six simple paths $\gamma_{i j}$ joining $x_{i}$ and $x_{j}$ that may only intersect at their endpoints.

The wished spherical surface $S$ is obtained by performing the surgery described in Lemma 3.23 (a) along these paths, gluing the sphere $S^{\prime}$ with $m_{i j}^{\prime}$ copies of $\mathbb{S}^{2} \backslash \operatorname{dev}_{\gamma_{i j}}$ along each $\gamma_{i j}$ for all $1 \leq i<j \leq 4$. This settles the case $\|\boldsymbol{m}\|_{1} \in 2 \mathbb{Z}$.

To treat the case when $\|\boldsymbol{m}\|_{1}$ is odd, it is enough to choose $\boldsymbol{m}^{\prime}=\boldsymbol{m}-\boldsymbol{e}_{\mathbf{1}}$ and to observe that $\boldsymbol{m}^{\prime} \in \Gamma^{4}$ and that $\boldsymbol{\vartheta}-\boldsymbol{m}^{\prime} \in[1,2] \times[0,1]^{3} \subset \Pi^{4}$. Then the above argument carries on.

Case (b): $m_{1}>m_{2}+m_{3}+m_{4}$ and $\|\boldsymbol{m}\|_{1}$ odd.

Since $\boldsymbol{\vartheta}-\boldsymbol{m}+\boldsymbol{e}_{\mathbf{1}} \in[1,2] \times[0,1] \times[0,1]^{2} \subset \Pi^{4}$ and $\boldsymbol{m}-\boldsymbol{e}_{\mathbf{1}}$ is even, we have $\boldsymbol{\vartheta}-\boldsymbol{m}+\boldsymbol{e}_{\mathbf{1}} \in \operatorname{int}\left(\Pi^{4} \cap \mathcal{A}^{4}\right)$ with no integral coordinate. Moreover, $\left(m_{1}-1\right)-m_{2}-m_{3}-m_{4}=2 d$ with $d \in \mathbb{Z}_{\geq 0}$. By Proposition 3.38 there exists a sphere $S^{\prime}$ with conical points $x_{1}, \ldots, x_{4}$ of angles $2 \pi \cdot\left(\boldsymbol{\vartheta}-\boldsymbol{m}+\boldsymbol{e}_{\mathbf{1}}\right)$; moreover, either $\gamma_{13}$ or $\gamma_{14}$ is a geodesic of length less than $\pi$, which we will denote by $\gamma$.

Hence, we can apply to $S^{\prime}$ the surgery operation described in Lemma 3.23(b) along $\gamma$, thus producing a sphere $S^{\prime \prime}$ with angles $2 \pi \cdot\left(\boldsymbol{\vartheta}-\boldsymbol{m}+(2 d+1) \boldsymbol{e}_{\mathbf{1}}\right)$.

Finally, we obtain our wished spherical surface by applying the operation of Lemma 3.23)(a) to $S^{\prime \prime}$ by gluing $m_{2}$ copies of $\mathbb{S}^{2} \backslash \operatorname{dev}_{\gamma_{12}}$ along $\gamma_{12}, m_{3}$ copies of $\mathbb{S}^{2} \backslash \operatorname{dev}_{\gamma_{13}}$ along $\gamma_{13}$, and $m_{4}$ copies of $\mathbb{S}^{2} \backslash \operatorname{dev}_{\gamma_{14}}$ along $\gamma_{14}$.

Case (c): $m_{1}>m_{2}+m_{3}+m_{4}$ with $m_{2}>0$ and $\|\boldsymbol{m}\|_{1}$ even.

We proceed analogously to case (b). Since $\boldsymbol{\vartheta}-\boldsymbol{m}+\boldsymbol{e}_{\mathbf{1 2}} \in[1,2] \times[1,2] \times[0,1]^{2} \subset \Pi^{4}$ and $\boldsymbol{m}, \boldsymbol{e}_{\mathbf{1 2}}$ are even, we have $\boldsymbol{\vartheta}-\boldsymbol{m}+\boldsymbol{e}_{\mathbf{1 2}} \in \operatorname{int}\left(\Pi^{4} \cap \mathcal{A}^{4}\right)$ with no integral coordinate. Moreover, $m_{1}-m_{2}-m_{3}-m_{4}=$ $2 d$ with $d \in \mathbb{Z}_{\geq 0}$. By Proposition 3.38 there exists a sphere $S^{\prime}$ with conical points $x_{1}, \ldots, x_{4}$ of angles $2 \pi \cdot\left(\boldsymbol{\vartheta}-\boldsymbol{m}+\boldsymbol{e}_{\mathbf{1 2}}\right)$; moreover, either $\gamma_{13}$ or $\gamma_{14}$ is a geodesic of length less than $\pi$, which we will denote by $\gamma$.

Hence, we can apply to $S^{\prime}$ the surgery operation described in Lemma 3.23(b) along $\gamma$, thus producing a sphere $S^{\prime \prime}$ with angles $2 \pi \cdot\left(\boldsymbol{\vartheta}-\boldsymbol{m}+\boldsymbol{e}_{\mathbf{1 2}}+2 d \boldsymbol{e}_{\mathbf{1}}\right)$.

Finally, we obtain our wished spherical surface by applying the operation of Lemma 3.23)(a) to $S^{\prime \prime}$ by gluing $\left(m_{2}-1\right)$ copies of $\mathbb{S}^{2} \backslash \operatorname{dev}_{\gamma_{12}}$ along $\gamma_{12}, m_{3}$ copies of $\mathbb{S}^{2} \backslash \operatorname{dev}_{\gamma_{13}}$ along $\gamma_{13}$, and $m_{4}$ copies of $\mathbb{S}^{2} \backslash \operatorname{dev}_{\gamma_{14}}$ along $\gamma_{14}$.

Case (d): $m_{1}>m_{2}+m_{3}+m_{4}$ with $m_{2}=0$ and $\|\boldsymbol{m}\|_{1}$ even.

Clearly, we must have $m_{1}=2+2 d$ with $d \in \mathbb{Z}_{\geq 0}$ and $m_{2}=m_{3}=m_{4}=0$ Hence, $\boldsymbol{\vartheta}-2 d \boldsymbol{e}_{\mathbf{1}}$ belongs to the interior of $\diamond_{\left(\frac{5}{2}, \frac{1}{2}, \frac{1}{2}, \frac{1}{2}\right)}$.

By Proposition 3.39, there exists a sphere $S^{\prime}$ with conical points $x_{1}, \ldots, x_{4}$ of angles $2 \pi \cdot(\boldsymbol{\vartheta}-$ $\left.2 d \boldsymbol{e}_{1}\right)$; moreover, $x_{1}$ and $x_{2}$ are joined by a smooth geodesic $\gamma$ of length strictly less than $\pi$.

Hence, we can apply to $S^{\prime}$ the surgery operation described in Lemma 3.23(b) along $\gamma$, thus 
producing a sphere $S$ with angles $2 \pi \cdot \boldsymbol{\vartheta}$.

In all cases, the spherical surface $S^{\prime}$ has non-coaxial holonomy by Proposition 3.38 in cases (a-b-c) and by Proposition 3.39 in case (d). Thus, the surface $S$ constructed performing gluing operations as in Lemma 3.23 is non-coaxial too.

\subsection{Splitting conical points}

The aim of this section is to complete the proof of Theorem $\mathrm{C}$, by showing the following.

Theorem 3.40 (Existence of spherical metrics for $n \geq 5$ ). Assume $n \geq 4$ and let $\vartheta_{1}, \ldots, \vartheta_{n}$ be real numbers that both the positivity constraints $(P)$ and the holonomy constraints (H) strictly. If $n=4$, then also assume that one $\vartheta_{i}$ is integral.

Then there exists a sphere $S$ endowed with a spherical metric with $n$ conical singularities of angles $2 \pi \vartheta_{1}, \ldots, 2 \pi \vartheta_{n}$ and non-coaxial holonomy. Moreover, such a metric is deformable.

Clearly, this immediately leads to our main result.

Proof of Theorem $[$. The statement for $n=3$ follows from Theorem 3.8 , since 3-punctured spheres are obtained by doubling spherical triangles. The statement for $n=4$ when all $\vartheta_{1}, \ldots, \vartheta_{4}$ are not integers has already been proven in Theorem 3.24 and for $n \geq 5$ is the content of Theorem 3.40 .

Notice that, if $n=4$ and $\boldsymbol{\vartheta}$ satisfies positivity and strict holonomy constraints, then at most one $\vartheta_{i}$ can be integer. This case is also taken care by Theorem 3.40 and so the proof is complete.

Theorem 3.40 is based on an inductive argument, whose key step can be formulated as follows.

Lemma 3.41 (Inductive step). Let $\vartheta \in \mathbb{R}_{+}^{n}$ and $M: \mathbb{R}^{n} \rightarrow \mathbb{R}^{n-1}$ be a merging operation of type $M_{(i+j)}$ or $M_{(i-j)}$, where $i, j \in\{1, \ldots, n\}$ are two distinct indices. If $M=M_{(i-j)}$, then assume that $\vartheta_{j} \notin \mathbb{Z}$.

Suppose that there exists a sphere $S^{\prime}$ endowed with a non-coaxial, angle-deformable spherical metric $g^{\prime}$ with $n-1$ conical singularities $x_{1}^{\prime}, \ldots, x_{n-1}^{\prime}$ of angles $2 \pi \cdot \boldsymbol{\vartheta}^{\prime}$, where $\boldsymbol{\delta}^{\prime}:=M(\boldsymbol{\delta})$ and $\boldsymbol{\delta}, \boldsymbol{\delta}^{\prime}$ are the defects associated to $\boldsymbol{\vartheta}, \boldsymbol{\vartheta}^{\prime}$. Then there exists a sphere $S$ endowed with a non-coaxial, angledeformable spherical metric $g$ with $n$ conical singularities of angles $2 \pi \cdot \vartheta$.

Proof. Since the metric $g$ is angle-deformable, there exists a neighbourhood $\mathcal{N}^{\prime} \subset \mathbb{R}^{n-1}$ of $\boldsymbol{\vartheta}^{\prime}$ and a continuous family of metrics $\mathcal{N}^{\prime} \ni \boldsymbol{\nu}^{\prime} \mapsto g_{\boldsymbol{\nu}^{\prime}}^{\prime}$ on $S^{\prime}$ such that $g_{\boldsymbol{\vartheta}^{\prime}}^{\prime}=g^{\prime}$ and $g_{\boldsymbol{\nu}^{\prime}}^{\prime}$ has singularities of angles $2 \pi \cdot \boldsymbol{\nu}^{\prime}$. Up to shrinking $\mathcal{N}^{\prime}$, we can assume that all $g_{\boldsymbol{\nu}^{\prime}}$ are $\varepsilon$-wide at $x_{n-1}^{\prime}$ and with non-coaxial holonomy.

Case $M=M_{(i+j)}$.

By Proposition 3.17, there exist an $|\eta|<\varepsilon / 2$ and an $\left(y_{1}, y_{2}\right)$-angle-deformable spherical triangle $\left(T, g^{\prime \prime}\right)$ with vertices $y_{1}, y_{2}, y_{3}$, angles $\pi\left(\vartheta_{i}, \vartheta_{j}, \vartheta_{i}+\vartheta_{j}-1+\eta\right)$, which is $\pi(1-\varepsilon / 2)$-wide at $y_{3}$. Thus, there exists a neighbourhood $\mathcal{N}^{\prime \prime}$ of $\left(\vartheta_{i}, \vartheta_{j}\right) \in \mathbb{R}^{2}$, a function $\theta_{3}: \mathcal{N}^{\prime \prime} \rightarrow \mathbb{R}$ with $\theta_{3}\left(\vartheta_{i}, \vartheta_{j}\right)=$ $\vartheta_{i}+\vartheta_{j}-1+\eta$ and a continuous family $\mathcal{N}^{\prime \prime} \ni \boldsymbol{\nu}^{\prime \prime} \mapsto g_{\nu^{\prime \prime}}^{\prime \prime}$ of spherical metrics on $T$ such that $g_{\left(\vartheta_{i}, \vartheta_{j}\right)}^{\prime \prime}=g^{\prime \prime}$ and $g_{\boldsymbol{\nu}^{\prime \prime}}^{\prime \prime}$ has conical angles $\pi\left(\nu_{1}^{\prime \prime}, \nu_{2}^{\prime \prime}, \theta_{3}\left(\boldsymbol{\nu}^{\prime \prime}\right)\right)$.

By continuity, there exists a neighbourhood $\mathcal{N}$ of $\boldsymbol{\vartheta} \in \mathbb{R}^{n}$ such that $\boldsymbol{\nu}^{\prime \prime}(\boldsymbol{\nu}):=\left(\nu_{i}, \nu_{j}\right) \in \mathcal{N}^{\prime \prime}$ and $\boldsymbol{\nu}^{\prime}(\boldsymbol{\nu}):=\left(\nu_{1}, \ldots, \widehat{\nu}_{i}, \ldots, \widehat{\nu}_{j}, \ldots, \nu_{n}, \theta_{3}\left(\nu_{i}, \nu_{j}\right)\right) \in \mathcal{N}^{\prime}$ for all $\boldsymbol{\nu} \in \mathcal{N}$.

For every such $\boldsymbol{\nu} \in \mathcal{N}$, consider the surface $\left(S, g_{\boldsymbol{\nu}}\right)$ obtained by gluing $\left(S^{\prime}, g_{\boldsymbol{\nu}^{\prime}(\boldsymbol{\nu})}^{\prime}\right)$ and the double of $\left(T, g_{\boldsymbol{\nu}^{\prime \prime}(\boldsymbol{\nu})}^{\prime \prime}\right)$ at the conical points $x_{n-1}^{\prime} \in S^{\prime}$ and $\left[y_{3}\right] \in D T$ according to Lemma 3.20. This construction provides a continuous family $\mathcal{N} \ni \boldsymbol{\nu} \mapsto g_{\boldsymbol{\nu}}$ of spherical metrics on $S$ with conical points of angles $2 \pi \cdot \boldsymbol{\nu}$. Moreover, the holonomy of $g_{\boldsymbol{\nu}}$ is non-coaxial, since it contains that of $g_{\boldsymbol{\nu}^{\prime}(\boldsymbol{\nu})}^{\prime}$, which is non-coaxial.

Case $M=M_{(i-j)}$.

Since $\delta_{j} \notin \mathbb{Z}$, we can apply Proposition 3.18 to obtain an $|\eta|<\varepsilon / 2$ and an $\left(y_{1}, y_{2}\right)$-angle-deformable spherical triangle $\left(T, g^{\prime \prime}\right)$ with vertices $y_{1}, y_{2}, y_{3}$, angles $\pi\left(\vartheta_{i}, \vartheta_{j}, \vartheta_{i}-\vartheta_{j}-1+\eta\right)$, which is $\pi(1-\varepsilon / 2)$ wide at $y_{3}$. The proof then works as in the previous case.

Finally, the argument is completed as follows. 
Proof of Theorem 3.40. Let $\boldsymbol{\delta}=\left(\vartheta_{1}-1, \ldots, \vartheta_{n}-1\right)$ as usual.

Case $n=4$ and $\vartheta_{i} \in \mathbb{Z}$.

Since $\boldsymbol{\delta} \in \operatorname{int}\left(\mathcal{A}^{4}\right)$, for any $j \neq i$ the operation $M=M_{(i+j)}$ satisfies $\boldsymbol{\delta}^{\prime}=M(\boldsymbol{\delta}) \in \operatorname{int}\left(\mathcal{A}^{3}\right)$ by Lemma 2.32. By Theorem 3.8 there exists a non-coaxial angle-deformable spherical triangle with angles $2 \pi\left(\delta_{1}^{\prime}+1, \delta_{2}^{\prime}+1, \delta_{3}^{\prime}+1\right)$ and so we can apply Lemma 3.41, thus obtaining the wished non-coaxial angle-deformable spherical surface of genus 0 with angles $2 \pi\left(\vartheta_{1}, \ldots, \vartheta_{4}\right)$.

Together with Theorem 3.24 , this settles the case $n=4$.

Case $n \geq 5$ : induction.

Assume now that the statement holds for $(n-1)$-punctured spheres: we will prove it for $n$-punctured spheres.

Since $n \geq 5$, by Theorem 2.27 there exists a merging operation $M$ such that $\boldsymbol{\delta}^{\prime}:=M(\boldsymbol{\delta})$ belongs to $\operatorname{int}\left(\mathcal{A}^{n-1}\right)$. By inductive hypothesis, there exists a surface $S^{\prime}$ of genus 0 with a non-coaxial angledeformable spherical metric and $n-1$ conical singularities of angles $2 \pi\left(\delta_{1}^{\prime}+1, \ldots, \delta_{n-1}^{\prime}+1\right)$. The conclusion now follows by Lemma 3.41

\section{List of symbols}

$\boldsymbol{e}_{\boldsymbol{i}} \quad \boldsymbol{i}$-th vector of the standard basis of $\mathbb{R}^{n}$

$1 \quad$ vector $\boldsymbol{e}_{\mathbf{1}}+\cdots+\boldsymbol{e}_{\boldsymbol{n}} \in \mathbb{R}^{n}$

$\boldsymbol{\vartheta}$ angle vector $\left(\vartheta_{1}, \vartheta_{2}, \ldots, \vartheta_{n}\right) \in$ $\mathbb{R}^{n}$

$\overline{\boldsymbol{\vartheta}} \quad$ reduced angle vector $\overline{\boldsymbol{\vartheta}} \in[0,2)^{n}$ with $\boldsymbol{\vartheta}-\overline{\boldsymbol{\vartheta}} \in 2 \mathbb{Z}$

$\boldsymbol{\delta} \quad$ defect vector $\boldsymbol{\vartheta}-\mathbf{1} \in \mathbb{R}^{n}$

$\overline{\boldsymbol{\delta}} \quad$ reduced defect vector $\overline{\boldsymbol{\vartheta}}-\mathbf{1} \in$ $[-1,1)^{n}$

$\mathcal{N} \quad$ small neighbourhood of $\boldsymbol{\vartheta}$ in $\mathbb{R}^{n}$

$\boldsymbol{\nu}$ angle vector in $\mathcal{N}$

$d_{1}(\cdot, \cdot) \quad$ standard $\ell^{1}$ distance in $\mathbb{R}^{n}$

$\|\cdot\|_{1} \quad$ standard $\ell^{1}$ norm in $\mathbb{R}^{n}$

$\mathbb{Z}_{o}^{n} \quad$ subset of odd-integral vectors, i.e. $\boldsymbol{m} \in \mathbb{Z}^{n} \subset \mathbb{R}^{n}$ with $\|\boldsymbol{m}\|$ odd

$\mathcal{H}^{n} \quad$ locus of $\delta \in \mathbb{R}^{n}$ such that $d_{1}\left(\boldsymbol{\delta}, \mathbb{Z}_{o}^{n}\right) \geq 1$

$\mathcal{P}^{n} \quad$ locus of $\bar{\delta} \in(-1,+\infty)^{n}$ such that $\sum_{i} \delta_{i}>-2$

$\mathcal{A}^{n} \quad$ intersection of $\mathcal{H}^{n}$ and $\mathcal{P}^{n}$

$M_{(i+j)} \quad$ algebraic positive merging operation

$M_{(i-j)} \quad$ algebraic negative merging operation

$\square^{n} \quad$ unit cube with integral vertices in $\mathbb{R}^{n}$

c center of a unit cube in $\mathbb{R}^{n}$

$\square_{c} \quad$ unit cube with center $c \in \mathbb{R}^{n}$

$\diamond^{n} \quad$ truncated cube $\square^{n} \cap \mathcal{H}^{n}$

$\diamond_{c} \quad$ truncated cube $\square_{c} \cap \mathcal{H}^{n}$ $\triangle_{\boldsymbol{c}}(\boldsymbol{m})$ half truncated cube with center $\boldsymbol{c}$ and vertex $\boldsymbol{m}$

$\boldsymbol{\delta}^{\pi} \quad$ radial projection of $\boldsymbol{\delta} \in \nabla_{\boldsymbol{c}}$ onto $\partial \diamond_{c}($ for $\boldsymbol{\delta} \neq \boldsymbol{c})$

$\mathbb{S}^{2}, \mathbb{S}^{3} \quad$ unit spheres endowed with the standard metric

$T^{1} \Sigma \quad$ unit tangent bundle to $\Sigma$

dev developing map of a simply connected surface

$\operatorname{dev}_{\gamma} \quad$ developing map of a path $\gamma$

$\dot{S} \quad$ complement of the conical points $x_{1}, \ldots, x_{n}$ in $S$

$\rho$ holonomy representation in $\mathrm{SO}(3, \mathbb{R})$

$\hat{\rho} \quad$ standard lift of the holonomy representation to $\mathrm{SU}(2)$

$c_{p} \quad$ constant loop based at the point $p$

$\gamma_{j} \quad$ loop that simply winds about the $j$-th marked point

$U_{j} \quad$ matrix in $\mathrm{SU}(2)$ representing the holonomy along $\gamma_{j}$

$v_{j} \quad$ vertex of a broken geodesic on $\mathbb{S}^{3}$

$s_{j} \quad$ side of a broken geodesic on $\mathbb{S}^{3}$

$\ell_{j} \quad$ length of the side $s_{j}$ of a broken geodesic on $\mathbb{S}^{3}$

$\left|x_{i} x_{i+1}\right| \quad$ length of the edge between $x_{i}$ and $x_{i+1}$ in a spherical polygon

$D S \quad$ surface obtained by doubling the surface with boundary $S$

$B_{\alpha}(r) \quad$ standard open $r$-neighbourhood of a vertex of angle $\pi \alpha$ in a spherical polygon 
$\bar{B}_{\alpha}(r) \quad$ standard closed $r$-neighbourhood of a vertex of angle $\pi \alpha$ in a spherical polygon

$S_{\alpha}(r) \quad$ standard open $r$-neighbourhood of a point of angle $2 \pi \alpha$ in a spherical surface

$\bar{S}_{\alpha}(r) \quad$ standard closed $r$-neighbourhood of a point of angle $2 \pi \alpha$ in a spherical surface

$B_{\alpha} \quad$ ordinary spherical bigon with angles $\pi \alpha$

$S_{\alpha} \quad$ double of $B_{\alpha}$

$B(d, \ell) \quad$ exceptional spherical bigon with angles $\pi d$ at distance $\ell$

$T(d, \ell, \alpha)$ spherical triangle with sides $\ell, \ell, 2 \pi d$ and angles $\pi \alpha, \pi(1-$ $\alpha), 2 \pi d$

$U_{y}(r) \quad$ complement in a spherical surface of the neighbourhood of the cone point $y$ of angle $2 \pi \alpha$ isometric to $B_{\alpha}(r)$

$S \#_{r} S^{\prime} \quad$ surface obtained by surgery at conical points

$S_{\gamma} \#_{\gamma^{\prime}} S^{\prime} \quad$ surface obtained by surgery along paths 


\section{References}

[1] Daniele Bartolucci, Francesca De Marchis, and Andrea Malchiodi, Supercritical conformal metrics on surfaces with conical singularities, Int. Math. Res. Not. IMRN (2011), no. 24, 5625-5643. MR 2863376

[2] Indranil Biswas, A criterion for the existence of a parabolic stable bundle of rank two over the projective line, Internat. J. Math. 9 (1998), no. 5, 523-533. MR 1644048 (99m:14063)

[3] Alexandre Eremenko, Metrics of positive curvature with conic singularities on the sphere, Proc. Amer. Math. Soc. 132 (2004), no. 11, 3349-3355 (electronic). MR 2073312 (2005h:53054)

[4] Alexandre Eremenko, Andrei Gabrielov, and Vitaly Tarasov, Metrics with conic singularities and spherical polygons, preprint arXiv:1405.1738.

[5] - Metrics with four conic singularities and spherical quadrilaterals, preprint arXiv:1409.1529.

[6] _ Spherical quadrilaterals with three non-integer angles, preprint arXiv:1504.02928.

[7] Daniel Gallo, Michael Kapovich, and Albert Marden, The monodromy groups of Schwarzian equations on closed Riemann surfaces, Ann. of Math. (2) 151 (2000), no. 2, 625-704. MR 1765706 (2002j:57029)

[8] Heinz Hopf, Zum Clifford-Kleinschen Raumproblem, Math. Ann. 95 (1926), no. 1, 313-339. MR 1512281

[9] Wilhelm Killing, Ueber die Clifford-Klein'schen Raumformen, Math. Ann. 39 (1891), no. 2, 257-278. MR 1510701

[10] Paul Koebe, Über die Uniformisierung beliebiger analytischer Kurven, Göttinger Nachrichten (1907), 191-210.

[11] _ Über die Uniformisierung beliebiger analytischer Kurven (Zweite Mitteilung), Göttinger Nachrichten (1907), 633-669.

[12] Feng Luo, Monodromy groups of projective structures on punctured surfaces, Invent. Math. 111 (1993), no. 3, 541-555. MR 1202134 (94b:32032)

[13] Robert C. McOwen, Point singularities and conformal metrics on Riemann surfaces, Proc. Amer. Math. Soc. 103 (1988), no. 1, 222-224. MR 938672 (89m:30089)

[14] Henri Poincaré, Sur l'uniformisation des fonctions analytiques, Acta Math. 31 (1908), no. 1, 1-63. MR 1555036

[15] Irina Scherbak, Rational functions with prescribed critical points, Geom. Funct. Anal. 12 (2002), no. 6, 1365-1380. MR 1952932 (2004c:14101)

[16] William P. Thurston, Shapes of polyhedra and triangulations of the sphere, The Epstein birthday schrift, Geom. Topol. Monogr., vol. 1, Geom. Topol. Publ., Coventry, 1998, pp. 511-549. MR 1668340 (2000b:57026)

[17] Marc Troyanov, Les surfaces euclidiennes à singularités coniques, Enseign. Math. (2) 32 (1986), no. 1-2, 79-94. MR 850552 (87i:30079)

[18] — Metrics of constant curvature on a sphere with two conical singularities, Differential geometry (Peñíscola, 1988), Lecture Notes in Math., vol. 1410, Springer, Berlin, 1989, pp. 296306. MR 1034288 (90m:53057)

[19] _ Prescribing curvature on compact surfaces with conical singularities, Trans. Amer. Math. Soc. 324 (1991), no. 2, 793-821. MR 1005085 (91h:53059) 